\title{
Neuropsychologische Testleistungen bei Patienten mit Koronarer Herzkrankheit \\ und ihr Zusammenhang mit morphologischen Auffälligkeiten im cranialen Kernspintomogramm
}

\author{
Dissertation \\ zur Erlangung des Doktorgrades \\ der Mathematisch-Naturwissenschaftlichen Fakultäten \\ der Georg-August-Universität zu Göttingen
}

\author{
vorgelegt von \\ Janka Koschack \\ aus Delmenhorst
}

Göttingen 2002 
D 7

Referent:

Prof. Dr. Eva Irle

Korreferent:

Prof. Dr. Gerd Lüer

Tag der mündlichen Prüfung: $\quad 30.01 .2003$ 
Wenn man einen Riesen sieht, so bestimme man erst den Stand der Sonne und gebe acht, ob es nicht nur der Schatten eines Zwerges ist.

(Novalis) 


\section{DANKSAGUNG}

Zunächst sei allen Patienten und Probanden gedankt, ohne deren Mitwirken die Untersuchung nicht möglich gewesen wäre.

Ich danke der Erstreferentin Frau Prof. Dr. Eva Irle für die Themenstellung und Betreuung während der Promotion und dem Korreferenten Herrn Prof. Dr. Gerd Lüer für die hilfreichen Anmerkungen bei der Fertigstellung der Arbeit.

Ganz herzlich bedanke ich mich auch bei meinen Diplomandinnen Dipl.-Psych. Annika Stechman und Dipl.-Psych. Nicole David, die mit großer Zuverlässigkeit und Selbständigkeit einen nicht unerheblichen Teil der neuropsychologischen Testungen dieser Studie durchführten.

Neben den an dieser Stelle namentlich ungenannt bleibenden Ärzten, Schwestern, Pflegern und Kardiotechnikern der Kardiologischen Stationen und der Thorax-, Herz- und Gefäßchirurgie gilt mein Dank PD Dr. med. Ivo Aleksic, der für mich Kontakte zu den Stationen herstellte und ein anregender Gesprächspartner war.

Ein herzliches Dankeschön gilt meinen Kollegen der Schwerpunktprofessur Neuropsychologie und Psychopathologie für ihre Bereitschaft zur wissenschaftlichen Diskussion. Besonders erwähnt sei an dieser Stelle Dipl.-Psych. Claudia Lange, ohne deren Vorarbeit die Auswertung der kernspintomographischen 3D-Datensätze nicht möglich gewesen wäre.

Ich möchte mich bei meinen Eltern bedanken, die mir die Ausbildung ermöglichten und stets bereit waren, mich in allen Belangen zu unterstützen.

Zahlreiche Rückschläge ließen mich immer wieder zweifeln, doch die Unterstützung meines Mannes ermöglichte es mir, weiterzumachen. Ihm gebührt deshalb der größte Dank. 


\section{INHALTSVERZEICHNIS}

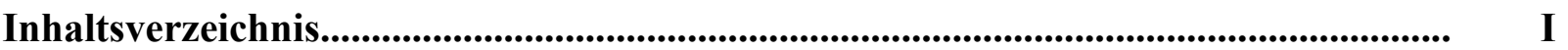

Verzeichnis der Tabellen.................................................................................................. IV

Verzeichnis der verwendeten Abkürzungen................................................................... V

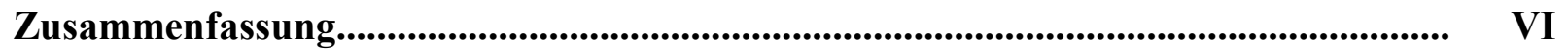

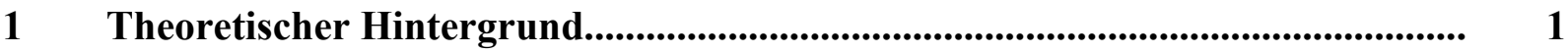

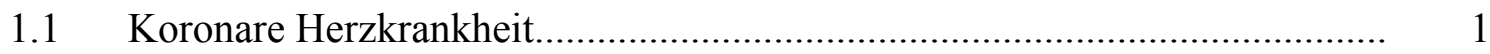

1.1.1 Epidemiologie ......................................................................................... 2

1.1.2 Funktionelle und morphologische Auswirkungen der Koronaren Herzkrankheit auf das Gehirn...

1.2 Erfassung kognitiver Funktionen im Rahmen klinisch-neuropsychologischer Forschung.

1.2.1 Erfassung intellektueller Fähigkeiten...............................................................

1.2.1.1 Empirische Befunde zu einzelnen HAWIE-R-Untertests......................... 5

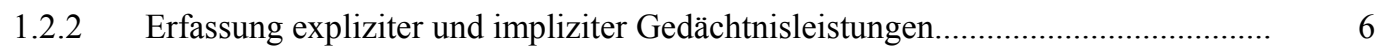

1.2.2.1 Empirische Befunde zu einzelnen WMS-R-Untertests............................... 7

1.2.3 Erfassung differentieller Leistungen in der Informationsverarbeitung................... 9

1.2.3.1 Empirische Befunde zu einzelnen klinischen Meßinstrumenten der Informationsverarbeitung.....

1.3 Kernspintomographische Diagnose von Gewebsschädigungen des Gehirns.. 12

1.4 White Matter Hyperintensitäten und lakunäre Infarzierung bei Patienten mit Koronarer Herzkrankheit..

1.4.1 White Matter Hyperintensitäten............................................................................. 14

1.4.1.1 Auftretenshäufigkeit von White Matter Hyperintensitäten und assoziierte Risikofaktoren.

1.4.1.2 Morphologische Korrelate der im Kernspintomogramm sichtbaren White Matter Hyperintensitäten.......

1.4.1.3 Klinische Relevanz von White Matter Hyperintensitäten......................... 18

1.4.2 Lakunäre Infarzierung.............................................................................. 20 
1.5 Pathologische Veränderungen des Temporallappens bei Patienten mit Koronarer Herzkrankheit.

1.5.1 Neuropathologische Veränderungen des Temporallappens..................................... 22

1.5.2 Kernspintomographisch erfasste Volumenreduktion von Temporallappenstrukturen.

1.5.2.1 Klinische Relevanz der kernspintomographischen Befunde.

1.5.3 Zusammenhang zwischen Alzheimer-Demenz und Koronarer Herzkrankheit........ 27

$1.6 \quad$ Fragestellung und Ableitung der Hypothesen............................................ 28

2 Methoden.

$2.1 \quad$ Patienten und Kontrollprobanden............................................................... 32

2.1.1 Patienten mit Koronarer Herzkrankheit.............................................................. 32

2.1.1.1 Rekrutierung.................................................................................... 32

2.1.1.2 Krankheitsbezogene Variablen.................................................... 33

2.1.2 Kontrollprobanden...................................................................................... 33

2.1.3 Vergleich der Patienten mit den Kontrollprobanden: Soziodemographische Variablen und Ausmaß der depressiven Symptomatik...

2.1.4 Vergleich der Patienten mit den Kontrollprobanden: Verteilung vaskulärer Risikofaktoren.

2.2 Untersuchungsverfahren.

2.2.1 Neuropsychologische Untersuchungsverfahren................................................. 37

2.2.1.1 Erfassung intellektueller Fähigkeiten................................................... 37

2.2.1.2 Erfassung expliziter und impliziter Lern- und Gedächtnisleistungen........ 38

2.2.1.3 Erfassung differentieller Leistungen in der Informationsverarbeitung..... $\quad 40$

2.2.2 Kernspintomographische Untersuchung........................................................... 43

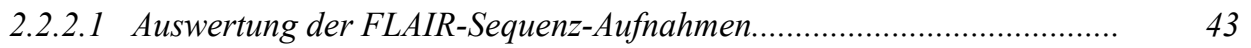

2.2.2.2 Läsionsanalyse lakunärer Infarzierungen................................................. 44

2.2.2.3 Auswertung der Volumendatensätze: Regions of Interest (ROIs)............. 44

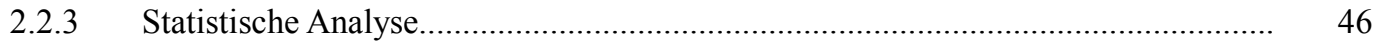

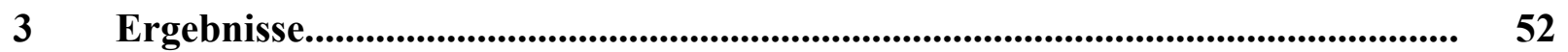

3.1 Neuropsychologische Untersuchungsverfahren........................................ 52

3.1.1 Vergleich der neuropsychologischen Testleistungen der Patienten und Kontrollprobanden.

3.1.2 Einfluß des intellektuellen Leistungsniveaus auf Gedächtnis- und Aufmerksamkeitsleistungen......

3.2 Kernspintomographische Untersuchung.

3.2.1 Schweregrade der White Matter Hyperintensitäten in der FLAIR-SequenzAufnahme

3.2.2 Lakunäre Infarzierung in der $T_{1}$-gewichteten Aufnahme 
3.2.3 Auswertung der Volumendatensätze.

3.2.3.1 Mittelwertsunterschiede in den Volumina verschiedener Hirnstrukturen

3.2.3.2 Korrelationen zwischen Volumina einzelner Temporallappenstrukturen

3.3 Zusammenhänge zwischen kernspintomographischen Auffälligkeiten und neuropsychologischen Testleistungen.

3.3.1 Zusammenhänge zwischen dem Schweregrad der White Matter Hyperintensitäten und neuropsychologischen Testleistungen

3.3.2 Zusammenhänge zwischen den Volumina temporaler Strukturen und neuropsychologischen Testleistungen.

4.1 Allgemeine Diskussion.

4.1.1 Neuropsychologische Testleistungen von Patienten mit Koronarer Herzkrankheit

4.1.2 Kernspintomographische Auffälligkeiten von Patienten mit Koronarer Herzkrankheit

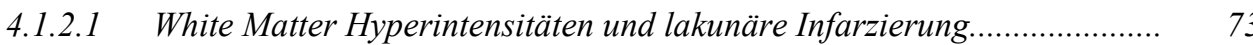

4.1.2.2 Volumenreduktion temporaler Strukturen.......................................... 77

4.1.3 Alternative Erklärungen für schlechtere kognitive Leistungen der Patienten............ 80

4.2 Abschließende Bemerkungen und Ausblick............................................ 81 


\section{VERZEICHNIS DER TABELLEN}

Tabelle $1 \quad$ Krankheitsbezogene Angaben zu den KHK-Patienten 33

Tabelle 2 Soziodemographische Variablen und Ausmaß der depressiven Sympto- 35 matik der Patienten und Kontrollprobanden

Tabelle 3 Verteilung vaskulärer Risikofaktoren bei Patienten und Kontrollproban- 36 den

Tabelle 4 Übersicht über die neuropsychologischen Untersuchungsverfahren 42

Tabelle 5 Neuropsychologische Testergebnisse der Patienten und Kontrollproban- 55 den

Tabelle 6 Verteilung der Schweregrade der White Matter Hyperintensitäten in den 58 mit der FLAIR-Sequenz aufgenommenen Kernspintomogrammen der Patienten und Kontrollprobanden

Tabelle 7 Häufigkeit und Lokalisation von Läsionen in der $\mathrm{T}_{1}$-gewichteten Auf- 59 nahme bei Patienten und Kontrollprobanden

Tabelle 8 Volumina verschiedener Hirnstrukturen in der Patienten- und Kontroll- 60 gruppe

Tabelle 9 Spearman-Rangkorrelationen zwischen Volumina einzelner Temporal- 61 lappenstrukturen in der Patienten- und Kontrollgruppe

Tabelle 10 Zusammenhänge zwischen dem Schweregrad der White Matter Hyperintensitäten und kognitiven Leistungen in der Patienten- und Kontrollgruppe

Tabelle 11 Zusammenhänge zwischen den Volumina temporaler Strukturen und neuropsychologischen Testleistungen in der Patientengruppe

\section{VERZEICHNIS DER ABBILDUNGEN}

Abbildung 1 Schweregrade der White Matter Hyperintensitäten $\quad 57$

Abbildung 2 Segmentierung des Hippocampus 


\title{
VERZEICHNIS DER VERWENDETEN ABKÜRZUNGEN
}

\author{
ACVB Aortokoronarer Venenbypass \\ BDI Beck Depressionsinventar \\ BMI Body Mass Index \\ DWM Deep White Matter \\ DWMH Deep White Matter Hyperintensitäten \\ HAWIE-R Hamburg-Wechsler-Intelligenztest für Erwachsene -revidierte Form- \\ KHK Koronare Herzkrankheit \\ MCI Mild Cognitive Impairment \\ MRT Magnetresonanztomographie \\ NFT Neurofibrillary Tangles \\ PET Positron Emissions Tomography \\ PTCA perkutane transluminale koronare Angioplastie \\ PVH Periventrikuläre Hyperintensitäten \\ ROI Region of Interest \\ SPECT Single Photon Emission Computerized Tomography \\ SPSS Statistical Package for the Social Sciences \\ SRTT Serielle Reaktionszeitaufgabe \\ TAP Testbatterie zur Aufmerksamkeitsprüfung \\ TIA Transiente ischämische Attacke \\ TMT Trail Making Test \\ WMH White Matter Hyperintensitäten \\ WMS-R Wechsler-Memory-Scale-Revised-
}




\section{ZUSAMMENFASSUNG}

Die mit der Koronaren Herzkrankheit assoziierten vaskulären Risikofaktoren erhöhen die Prävalenz für mikroangiopathische Erkrankungen des Gehirns: Kernspintomographische Untersuchungen zeigten bei Patienten mit Koronarer Herzkrankheit vermehrt lakunäre Infarzierungen und höhergradige Veränderungen der White Matter. Die negativen Auswirkungen der Koronaren Herzkrankheit auf die kognitive Leistungsfähigkeit der Patienten wurden in neuropsychologischen Studien ebenfalls nachgewiesen, so daß Patienten mit Koronarer Herzkrankheit als Risikogruppe für die Entwicklung einer vaskulären Demenz betrachtet wurden. In jüngster Zeit zeigten jedoch neuropathologische Studien, daß die morphologischen Veränderungen in Gehirnen von Patienten mit Koronarer Herzkrankheit nicht nur mikroangiopathische Prozesse abbilden, sondern auch Veränderungen im Temporallappen insbesondere im Hippocampus beinhalten, die bisher als typisch für Patienten mit Alzheimer-Demenz galten.

In der vorliegenden Arbeit wurden die Auswirkungen der Koronaren Herzkrankheit auf das Gehirn und die kognitive Leistungfähigkeit untersucht. Patienten mit Koronarer Herzkrankeit wurden mit gesunden Kontrollprobanden bezüglich kernspintomographisch identifizierbarer Auffälligkeiten wie Anzahl lakunärer Infarzierungen, Schwere der Veränderungen in der White Matter und Volumenreduktionen bestimmter Hirnregionen sowie neuropsychologischer Testleistungen verglichen. Korrelationsanalysen sollten Aufschluß über die psychologische Relevanz der morphologischen Veränderungen geben.

Es konnten deutliche Leistungsdefizite der Patienten im Bereich des expliziten Gedächtnisses, der impliziten Lerngeschwindigkeit und des schlußfolgernden Denkens nachgewiesen werden. In beiden Gruppen wurden mikroangiopathische Veränderungen im Gehirn kernspintomographisch identifiziert, die deutlich mit höherem Lebensalter assoziiert waren und in der Patienten- wie auch Kontrollgruppe in einem negativen Zusammenhang mit der kognitiven Leistungsfähigkeit standen. Zusätzlich zu diesen unspezifischen mikroangiopathischen Auffälligkeiten wurden in der vorliegenden Studie erstmals in vivo morphologische Veränderungen des Temporallappens bei Patienten mit Koronarer Herzkrankheit gezeigt: Das Volumen des Hippocampus war beidseitig deutlich reduziert und mit kleineren Volumina weiterer temporaler Strukturen sowie schlechteren neuropsychologischen Testleistungen assoziiert. 


\subsection{KORONARE HERZKRANKHEIT}

In westlichen Industrienationen stellen Erkrankungen des Herz-Kreislauf-Systems die Haupttodesursache dar. Die häufigste dieser Erkrankungen ist die Koronare Herzkrankheit (KHK), unter der in der Bundesrepublik Deutschland derzeit ca. 5-10\% der männlichen Bevölkerung leiden. Ursache der KHK ist eine Sklerose der den Herzmuskel versorgenden Kranzarterien. Durch Ablagerung von Lipiden, Bindegewebe und Kalk kommt es zu einer Einengung der arteriellen Strombahn (=Stenose). Diese Stenose bleibt anfänglich klinisch stumm, erst bei einer kritischen Einengung des Gefäßquerschnitts um etwa 70\% kommt es zu einer klinisch relevanten Ischämie des Myokards (=Herzmuskelwand). Diese Substrat- und Sauerstoffmangelversorgung des Herzmuskels führt zu den für die KHK typischen Symptomen: stabile und instabile Angina pectoris, akuter Myokardinfarkt, Herzrhythmusstörungen einschließlich plötzlichen Herztodes, Herzinsuffizienz (Grädel \& Schulte, 1992).

Die gängigste Einteilung der KHK erfolgt in Abhängigkeit von Ausmaß und Lokalisation der betroffenen Herzkranzgefäße. Dabei werden die rechte Kranzarterie sowie der Ramus interventricularis anterior und der Ramus circumflexus der linken Kranzarterie je als einzelnes Gefäß aufgefaßt. Demnach können 1-, 2- oder 3-Gefäß-KHK unterschieden werden. Eine Stenose des Hauptstamms der linken Kranzarterie ist selten, aber besonders gefährlich, da bei einem akuten Verschluß die gesamte Blutversorgung ausfällt. Die Therapie der ersten Wahl ist zunächst unabhängig von der Anzahl der betroffenen Gefäße eine konservative, d.h. es erfolgt eine individuelle Einstellung der Patienten mit Medikamenten der Klassen Antihypertensiva, Koronarmittel, Diuretika, Lipidsenker und Antikoagulantien. Eine weitere nichtoperative Therapie stellt die sogenannte perkutane transluminale koronare Angioplastie (PTCA) dar, bei der mittels eines intrakoronar eingeführten Ballonkatheters das verengte Gefäß dilatiert wird. Auch ein bereits stattgefundener Herzinfarkt ist keine hinreichende Bedingung für eine invasive Intervention. Lediglich bei Vorliegen einer medikamentös nicht beherrschbaren Angina pectoris, einer Hauptstammstenose der linken Kranzarterie oder einer 3Gefäß-KHK mit positivem Belastungs-EKG und eingeschränkter linksventrikulärer Funktion ist der aortokoronare Venenbypass (ACVB) indiziert. 


\subsubsection{EPIDEMIOLOGIE}

Die Rolle prädisponierender genetischer Faktoren ist unumstritten. Als nicht gesichert hingegen gilt die Annahme von psychosozialen und persönlichkeitsabhängigen Risikofaktoren, z.B. dem sogenannten "Typ-A"-Verhalten (Ragland \& Brand, 1988). Epidemiologische Studien haben eine Reihe von Risikofaktoren für die Entwicklung einer Koronarsklerose aufzeigen können, die vor allem in ihrer Kombination die Wahrscheinlichkeit, an einer KHK zu erkranken, beträchtlich erhöhen (Schanzenbächer \& Kochsiek, 1993):

Hypercholesterinämie: Ernährungsgewohnheiten in vielen Industrienationen haben in den letzten Jahrzehnten zu einem Anstieg des Cholesterinspiegels geführt, der mit einem erhöhten kardiovaskulären Todesrisiko assoziiert ist. So ist beispielsweise bei Männern mit einem erhöhten Cholesterinspiegel, d.h. > 240mg/\%, das Risiko einer KHK signifikant erhöht.

Arterielle Hypertonie: Bluthochdruck, d.h. ein systolischer Blutdruck von $>140 \mathrm{~mm} \mathrm{Hg}$ und ein diastolischer Blutdruck von $>90 \mathrm{~mm} \mathrm{Hg}$, gilt als gesicherter Risikofaktor für die Entwicklung einer Koronarsklerose, vor allem in Kombination mit Hypercholesterinämie und Nikotinabusus.

Diabetes mellitus: Diese Erkrankung führt zu diffusen arteriosklerotischen Veränderungen des gesamten vaskulären Systems. Ob bereits eine pathologisch erhöhte Glucosetoleranz einen Risikofaktor darstellt, ist noch nicht geklärt.

Zigarettenkonsum: Ein Nikotinabusus von mehr als 20 Zigaretten pro Tag erhöht das Risiko eines Herzinfarktes um das Dreifache. Bei Abstinenz sinkt das Risiko zwar, erreicht aber niemals wieder das Niveau von Nichtrauchern.

Adipositas: Als klinisch relevantes Übergewicht gilt ein Verhältnis von Körpergewicht in $\mathrm{kg}$ zu Körpergröße in $\mathrm{m}^{2}>30$ (Bray, 1978). Neben diesem Body Mass Index spielt auch die Fettverteilung eine Rolle. Besonders die für Männer typische Verteilung des Körperfetts im Oberbauchbereich erhöht die Auftretenswahrscheinlichkeit vaskulärer Risikofaktoren und somit auch der KHK. 


\subsubsection{FUNKTIONELLE UND MORPHOLOGISCHE AUSWIRKUNGEN DER KORONAREN HERZKRANKHEIT AUF DAS GEHIRN}

Der Einsatz der für cerebrale Läsionen sensitiven Kernspintomographie in Untersuchungen mit großer Probandenzahl zeigte, daß neurologisch unauffällige Personen mit zunehmendem Alter ein erhöhtes Risiko für cerebrale Auffälligkeiten aufweisen. Diese Auffälligkeiten stellen sich kernspintomographisch als Hyperintensitäten in der weißen Substanz und lakunäre Infarzierungen dar. Neben dem Alter kristallisierten sich vaskuläre Risikofaktoren wie z.B. Hypertonie und damit verbunden das Vorliegen einer Koronaren Herzkrankheit als Prädiktoren dieser kernspintomographisch sichtbaren Auffälligkeiten heraus.

Epidemiologische und prospektive Studien zeigen, daß das Vorliegen kardiovaskulärer Risikofaktoren und Erkrankungen nicht nur mit schlechteren kognitiven Leistungen assoziiert ist (Breteler, Claus, Grobbee \& Hofman, 1994). Darüber hinaus erhöhen sie das Risiko einer dementiellen Erkrankung, und zwar nicht nur das Risiko für die Entwicklung einer vaskulären Demenz, sondern auch für die Entwicklung einer Alzheimer-Demenz (Breteler, Swieten et al., 1994; Skoog et al., 1996). Aktuelle quantitative kernspintomographische Untersuchungen zeigen bei Patienten mit vaskulärer Demenz eine Volumenreduktion in medialen Temporallappenstrukturen, insbesondere im Hippocampus. Diese Volumenreduktion der hippocampalen Formation geht mit neuropathologischen Auffälligkeiten einher, die bisher als typisch für die Alzheimer-Demenz galten.

Es deutet sich an, daß die bisher gezogene diagnostische Trennung von vaskulärer und Alzheimer-Demenz in dieser Schärfe nicht aufrechtzuerhalten ist und vaskuläre Risikofaktoren sowie das Vorliegen einer KHK auch bei der Entwicklung einer Alzheimer-Demenz eine Rolle spielen.

\subsection{ERFASSUNG KOGNITIVER FUNKTIONEN IM RAHMEN KLINISCH- NEUROPSYCHOLOGISCHER FORSCHUNG}

Viele der Testverfahren, die in der klinischen Neuropsychologie eingesetzt werden, wurden ursprünglich entwickelt, um "normale" und "gesunde" kognitive Funktionen des Menschen zu erfassen. Auch Testverfahren experimenteller Natur, die für grundlagenorientierte Studien über spezifische Dysfunktionen des Gehirns kreiert wurden, fanden häufig Eingang in die klinische Neuropsychologie. In beiden Fällen lag die Basis für die Entwicklung dieser Testverfahren in (neuro-) psychologischen Theorien über die Struktur und die Funkti- 
onsweise des menschlichen Verhaltens und des menschlichen Gehirns. Klinisch orientierte neuropsychologische Studien nehmen eine Mittelstellung zwischen klinischneuropsychologischer Einzelfalldiagnostik und experimentell-neurowissenschaftlicher Grundlagenforschung ein. Im Rahmen solcher Studien geht es weder um die einzelfallorientierte Diagnose kognitiver Störungen noch um die Überprüfung von theoretischen Annahmen über Funktionen des menschlichen Verhaltens oder des menschlichen Gehirns. Vielmehr geht es um die Überprüfung spezifischer Annahmen über z.B. die Ätiologie einer Erkrankung oder um die Gemeinsamkeiten zwischen verschiedenen Störungsbildern. Im Rahmen dieser Studien haben sich verschiedene Testverfahren zur Erfassung kognitiver Funktionen etabliert, die sich im wesentlichen in drei Bereiche einteilen lassen: Neben der Erfassung mentaler oder intellektueller Fähigkeiten spielt die Messung expliziter und impliziter Gedächtnisleistungen eine Rolle ebenso wie die Erhebung von differentiellen Leistungen in der Informationsverarbeitung.

\subsubsection{ERFASSUNG INTELLEKTUELLER FÄHIGKEITEN}

In den Anfängen der psychometrischen Erfassung intellektueller Fähigkeiten des Menschen wurde Intelligenz als einheitliche Größe betrachtet, die ebenso wie z.B. die Körpergröße in regelhafter und stetiger Weise im Rahmen der kindlichen Entwicklung anwächst (vgl. Binet \& Simon, 1908) und deren Abfall in ebenso stetiger Weise mit dem Verlust von Hirngewebe assoziiert ist (vgl. Lashley, 1938). Neuropsychologische Untersuchungen u.a. zeigten jedoch, daß Intelligenz keine einheitliche biologische Größe ist, sondern Intelligenztests verschiedene spezifische kognitive Funktionen messen und diese lediglich zu einer einheitlichen Größe, dem Intelligenzquotienten, zusammenfassen. Desweiteren wurde festgestellt, daß intellektuelle Leistungen mit der Funktion verschiedener Hirnregionen assoziiert sind und nicht auf eine einzelne Hirnregion zurückgeführt werden können. So zeigte z.B. Hebb in Untersuchungen mit frontalhirngeschädigten Patienten, daß diese keineswegs unterdurchschnittliche IQ-Werte aufwiesen, obwohl bis dato der Frontallappen als Sitz der generellen Intelligenz galt (Hebb \& Penfield, 1940). In der Entwicklung von Intelligenztests wurden die neuen Erkenntnise verarbeitet. So folgte beispielsweise auch Wechsler bei der Konstruktion seiner AdultIntelligence-Scale dem neuen Konzept (Wechsler, 1955). Nach Matarazzo ist die am häufigsten zitierte Wechslersche Definition von Intelligenz folgende: "Intelligenz ist ein hypothetisches Konstrukt, ist die zusammengesetzte Fähigkeit des Individuums, zielgerichtet zu handeln, rational zu denken und sich wirkungsvoll mit seiner Umwelt auseinanderzusetzen. Sie ist zusammengesetzt oder global, weil sie aus Elementen oder Fähigkeiten besteht, die, ob- 
wohl nicht völlig unabhängig, qualitativ unterscheidbar sind." (Matarazzo, 1982, S. 121, zitiert nach Tewes, 1991, S.5). Der Hamburg-Wechsler-Intelligenztests für Erwachsene Revision (HAWIE-R) (Tewes, 1991) ist die deutschen Version der Wechsler Adult Intelligence Scale - Revised (WAIS-R) (Wechsler, 1981). Er eignet sich als Testbatterie besonders für den klinischen Einsatz: Der Gesamt-IQ wird im Gegensatz zu anderen Intelligenztests wie z.B. beim Raven-Matrizen-Test (Kratzmeier \& Horn, 1987) additiv über die Zusammenfassung der in verschiedenen Untertests erreichten Wertpunkte ermittelt, die differentielle intellektuelle Leistungen erfassen. Er erlaubt somit neben der Angabe eines Gesamt-IQ auch die klinisch-diagnostisch wertvollere Darstellung eines intellektuellen Leistungsprofils. Es ist durchaus üblich, lediglich einzelne Untertests des HAWIE-R durchzuführen, die für die jeweilige Fragestellung interessant sind.

\subsubsection{Empirische Befunde zu einzelnen HAWIE-R-Untertests}

Allgemeines Wissen: Dieser Untertest fragt überlerntes Schul- und Weltwissen ab und spiegelt die kulturellen Erfahrungen und die Aufgeschlossenheit des Individuums der Umwelt gegenüber wieder (Zimmerman, Woo-Sam \& Glasser, 1973). Da Leistungen in diesem Test sich als relativ stabil gegenüber traumatischen Hirnverletzungen herausstellten (Russell, 1987), wird er im Rahmen klinischer Fragestellungen häufig als Schätzer der prämorbiden Fähigkeit eingesetzt.

Gemeinsamkeitenfinden: Dieser Untertest erfaßt die Fähigkeit des Indiviuums, auf der Basis von Abstraktion und Generalisation verbale Konzepte zu bilden. Leistungen in diesem Test sind funktionell mit linkstemporalen und frontalen Hirnregionen assoziiert (Chase et al., 1984; Warrington, James \& Maciejewski, 1986). Als Operationalisierung der verbalen Konzeptbildung scheint der Untertest ein guter Prädiktor für den Verlust kognitiver Fähigkeiten im Rahmen eines dementiellen Prozesses zu sein (Rue \& Jarvik, 1987).

Bilderergänzen: Dieser Untertest überprüft die Fähigkeit, visuelles Material zu organisieren und schlußfolgernd zu denken. Leistungen in diesem Test scheinen relativ stabil gegenüber traumatischen Hirnschädigungen zu sein und zwar unabhängig davon, ob es sich um eine rechts- oder linksseitige Schädigung handelt (Crosson, Greene \& Roth, 1990). Robust erscheint er ebenfalls gegenüber Abbauprozessen bei Alzheimer-Patienten, die in milden bis moderaten Krankheitsstadien noch keine Defizite in diesem Test zeigen (Logsdon, Teri, Williams, Vitiello \& Prinz, 1993). Hingegen erwies er sich bei pathologischen Prozessen, die subcorticale Strukturen involvieren, als sensitiv (Cummings \& Huber, 1992). 
Mosaiktest: Dieser Untertest überprüft visuokonstruktorische Fähigkeiten, d.h. Formen wahrzunehmen, zu analysieren und zu zerlegen. Diese Leistungen sind mit der Funktion der posterioren Parietalregion vor allem der rechten Hemisphäre verknüpft (Chase et al., 1984). Diese Assoziation wird unterstützt von Ergebnissen aus Läsionsstudien, die Patienten mit posteroparietalen Schädigungen untersucht haben (vgl. Warrington et al., 1986). Da zur Bewältigung des Tests im weiteren Sinne auch Problemlösestrategien angewendet werden müssen, überrascht es nicht, daß Patienten mit schweren Schädigungen der Frontalregion defizitäre Leistungen erbringen. Ihre Schwierigkeiten scheinen weniger in einer defizitären Visuokonstruktion begründet als vielmehr in dem Verlust kognitiver Flexibilität (Johanson, Gustafson \& Risberg, 1986). Im Rahmen hirnorganischer Abbauprozesse zeigen Patienten bereits in frühen Stadien Beeinträchtigungen in diesem Test (Larrabee, Largen \& Levin, 1985). Eine niedrige Anzahl erreichter Punkte kann ein Hinweis auf eine beginnende konstruktive Apraxie sein. Eine generelle Verlangsamung psychomotorischer Leistungen muß jedoch auch in Erwägung gezogen werden, da der Test sowohl eine Power- als auch eine Speedkomponente enthält.

\subsubsection{ERFASSUNG EXPLIZITER UND IMPLIZITER GEDÄCHTNISLEISTUNGEN}

Seit den Ergebnissen von Scoville und Milner (1957), die die Dissoziation expliziter/deklarativer und impliziter/nondeklarativer Lern- und Gedächtnissysteme zeigen konnten, gilt die (mind.) duale Natur von Lern- und Gedächtnisprozessen als allgemein anerkannt: Ihr mittlerweile berühmter Patient H.M. war nach einer bilateralen mediotemporalen Resektion nicht mehr in der Lage, neue Gedächtnisinhalte zu speichern, und wies profunde Defizite auch im Abruf biographischen Materials auf. Trotz dieser Defizite im expliziten Lern- und Gedächtnissystem konnte er neue Fähigkeiten über implizite und prozedurale Prozesse lernen, z.B. zeigte er deutliche Verbesserungen im Spiegelschreiben, obwohl er sich nicht daran erinnern konnte, den Test bereits mehrmals absolviert gehabt zu haben. Es existieren mittlerweile verschiedene Modelle multipler Gedächtnissysteme, die noch weitere Unterteilungen der beiden Systeme annehmen (vgl. Markowitsch, 1996; Squire, 1986; Tulving, 1984). So differenziert Markowitsch z.B. zwischen zwei deklarativen (expliziten) und zwei nondeklarativen (impliziten) Speichern. Er grenzt das episodische, autobiographische Gedächtnissystem, das singuläre Ereignisse auf einer Raum-Zeit-Achse anordnet, von dem semantischen oder Wissens- und Faktengedächtnis ab, in dem allgemeines Weltwissen und grammatische Regeln gespeichert werden. Diesen beiden deklarativen Systemen stehen zwei implizite/nondeklarative gegenüber: das prozedurale Gedächtnis, unter dem erlernte Bewegungsab- 
läufe oder Handlungsstrategien summiert werden, und das Priming, d.h. der Einfluß vorgeschalteter (subliminaler) Sinneseindrücke auf die Informationsverarbeitung (Markowitsch, 1996). Diese Dissoziationen sind neuronanatomisch belegt: Während explizite/deklarative Einspeicherungs- und Abrufprozesse eher mit mediotemporalen und präfrontalen Hirngebieten assoziiert sind, scheinen bei nondeklarativen Prozessen z.B. beim implizit-motorischen Lernen eher subkortikale und primär-motorische Hirnregionen involviert zu sein (für einen Überblick, s. Cabeza \& Nyberg, 2000).

Ein mittlerweile anerkanntes experimentelles Paradigma zur Erfassung impliziter (motorischer) Gedächtnisleistungen stellt die Serial Reaction Time Task (SRTT) dar, die Nissen und Bullemer 1987 einführten. Es existieren verschiedene Modifikationen der Originalaufgabe. Der Grundaufbau ist folgender: An vier feststehenden Positionen des Bildschirms werden Stimuli (Sternchen) präsentiert. Der Proband soll so schnell wie möglich auf die korrespondierende Reaktionstaste drücken. Er hat keine Kenntnis davon, daß es sich bei der Abfolge der Positionen um eine wiederkehrende Sequenz handelt, die wiederholt über sehr viele Versuchdurchgänge dargeboten wird. Das Ausmaß des impliziten Lernens wird durch den Abfall der Reaktionszeiten operationalisiert. Daran wird deutlich, daß der Proband von dem implizit erworbenen Wissen profitiert (zu empirischen Befunden zur SRTT: s. Abschnitt 1.4.2).

Die Wechsler Memory Scale-Revised (WMS-R) (Wechsler, 1987) hingegen erlaubt die Erfassung klinisch relevanter Störungen des deklarativen/expliziten Gedächtnisses bezüglich ihres Schweregrades als auch ihrer Modalitätsspezifität. Die getesteten Funktionen umfassen das deklarative Gedächtnis für verbales und visuelles Material, wobei in neun Untertests bedeutungshaltige und abstrakte Reize verwendet werden und der direkte sowie der verzögerte Abruf überprüft werden.

\subsubsection{Empirische Befunde zu einzelnen WMS-R-Untertests}

Logical Memory I und II (sofortiger u. verzögerter Abruf): Diese Untertests überprüfen die Güte der Einspeicherung und des verzögerten Abrufs von verbalem Material in Form von zwei kurzen, verbal präsentierten Geschichten. Sie werden in klinischen Fragestellungen zur Überprüfung von Lateralisierungseffekten vor allem im Rahmen der prächirurgischen Epilepsiediagnostik eingesetzt. Die Ergebnisse sind widersprüchlich. In einigen Studien werden Leistungsunterschiede zwischen links- und rechtsseitig geschädigten Patienten gefunden (zB. Chelune \& Bornstein, 1988; Delany, Rosen, Mattson \& Novelly, 1980), in anderen hingegen nicht (z.B. Loring, Lee, Martin \& Meador, 1989). Neben der Überprüfung von Lateralisie- 
rungseffekten eignen sich Logical Memory I und II besonders zur Frühdiagnostik mnestischer Defizite im Rahmen dementieller Syndrome. Bezüglich dieser Fragestellung zeigen eine Vielzahl von Studien den differentiellen und auch prognostischen Wert (z.B. Chen et al., 2001; McKhan et al., 1984; Small, Fratiglioni \& Bäckman, 2001).

Verbal Paired Associates I und II (sofortiger u. verzögerter Abruf): Diese Untertests überprüfen die Fähigkeit, verbal präsentierte Wortpaare assoziativ miteinander verknüpfen und abrufen zu können. Sie haben im Rahmen lateralitätsspezifischer Fragestellungen einen hohen diskriminativen Wert bei der Unterscheidung links- versus rechtsseitig geschädigter Patienten, und zwar unabhängig von der Ätiologie der Schädigung (Chelune \& Borstein, 1988; Vakiel, Hoofien \& Blachstein, 1992). Auch bei Patienten mit Gedächtnisproblemen auf der Basis dementieller Abbauprozesse zeigt sich das verbale Assoziationslernen diskriminativ gegenüber gesunden und depressiven Kontrollprobanden (Squire \& Shimamura, 1986).

Visual Paired Associates I und II (sofortiger u. verzögerter Abruf): Diese Untertests überprüfen die Fähigkeit, eine bestimmte Farbe mit einem Muster assoziativ verknüpfen und abrufen zu können. Das Material wird nonverbal präsentiert. Untersuchungen haben gezeigt, daß dieser Test nicht eine analoge Abbildung der verbalen Variante (s.o.) mit nonverbalem Material ist. Vielmehr konnte nachgewiesen werden, daß Gesunde wie auch Patienten das Materal verbalisieren und verbale Assoziationen bilden. Dementsprechend ist das visuelle Paarassoziationslernen nicht zur Überprüfung von Lateralisierungseffekten geeignet (Chelune \& Bornstein, 1988). Bei Demenz-Patienten hingegen konnten deutliche Defizite nachgewiesen werden, die bereits bei der Einspeicherung erhebliche Probleme zeigten (Butters, Salmon \& Cullum, 1988).

Visual Reproduction I und II (sofortiger und verzögerter Abruf): Diese Untertests überpüfen die Fähigkeit, nonverbal präsentiertes Material in Form von geometrischen Mustern einzuspeichern und abzurufen. Lateralisierungseffekte konnten weder für den sofortigen noch für den verzögerten Abruf konsistent gezeigt werden (vgl. Chelune \& Bornstein, 1988). Dies wird wie auch beim Untertest Visual Paired Associates auf die Verbalisierungsmöglichkeit des zu lernenden Materials zurückgeführt. Im Rahmen von demenzdiagnostischen Fragestellungen hingegen zeigten sich Defizite in der visuelle Reproduktion bei Patienten mit Alzheimer-Demenz (z.B. Jacobs, Tröster \& Butters, 1990).

Figural Memory: Dieser Untertest überprüft die Fähigkeit, nach kurzer Präsentation abstrakt gemusterte Vierecke einzuspeichern und unmittelbar danach aus einer Reihe von an- 
deren zu identifizieren. Obgleich der Versuch, Verbalisierungsmöglichkeiten zu vermeiden und einen nonverbalen Gedächtnistest zu entwickeln, als gelungen betrachtet werden kann, diskriminiert der Test nicht zwischen rechts- und linksseitig geschädigten Patienten (vgl. Lezak, 1995). Dennoch konnten Korrelationsstudien zeigen, daß gerade in Patientengruppen Leistungen in diesem Test mit anderen nonverbalen Testleistungen zusammenhingen (Bornstein \& Chelune, 1988).

Zusätzlich zu den beschriebenen Tests zum deklarativ/expliziten Gedächtnis überprüfen fünf weitere Untertests der $W M S-R$ aufmerksamkeitsabhängige Leistungen des Kurzzeitgedächtnisses (Mental Control, Digit Span Forward u. Backward, Visual Memory Span Forward u. Backward). Seitdem Miller (1956) nachwies, daß das Kurzzeitgedächtnis einer deutlichen Kapazitätsbeschränkung unterliegt und diese Beschränkung mit erheblichen Konsequenzen für alle Prozesse der Wahrnehmung, Verarbeitung und Speicherung von Information verbunden ist, wurde das Modell des Kurzzeitgedächtnisses immer weiter elaboriert (z.B. Baddeley, 1992). Innerhalb dieser modernen Konzeptionen wird die Trennung von Kurzzeitgedächtnis- und Aufmerksamkeitsprozessen immer schwieriger (Sohlberg \& Mateer, 1987); auch neuroanatomisch gibt es Belege für die funktionelle Überlappung dieser Prozesse (LaBar, Gitelman, Parrish \& Mesulam, 1999). In praktischen und vor allem klinischen Bezügen wird die Kurzzeitgedächtnisspanne mit der Aufmerksamkeitsspanne gleichgesetzt: Heute werden Tests mit hohen Anfordeungen an das Arbeitsgedächtnis, z.B. das Zahlennachsprechen (Digit Span, WMS-R), als Aufmerksamkeitstests eingeordnet (vgl. Lezak, 1995, S. 29 und S. 356 ff.) (s. Abschnitt 1.2.3).

\subsubsection{ERFASSUNG DIFFERENTIELLER LEISTUNGEN IN DER INFORMATIONSVERARBEI- TUNG}

Es gibt bis heute keine allgemein anerkannte Definition, welche kognitiven Prozesse oder Zustände unter dem Begriff Aufmerksamkeit subsummiert werden können. Frühere, mittlerweile eher historische Definitionen beschränkten sich auf ganz bestimmte spezifische Aufmerksamkeitsleistungen. Moderne Theorien zur Aufmerksamkeit haben gemeinsam, daß sie den Begriff Aufmerksamkeit nicht mehr verwenden, sondern allgemein von Informationsverarbeitung sprechen und darunter bestimmte Kapazitäten und Prozesse zusammenfassen, die bestimmen, in welcher Weise ein Individuum Reize wahrnimmt und verarbeitet. Es wird davon ausgegangen, daß die Informationsverarbeitung einer Kapazitätsbeschränkung unterliegt, die dazu führt, daß simultane und gleichartige Anforderungen an das System zu Interferenzen führen. Informationsverarbeitung wird 
renzen führen. Informationsverarbeitung wird weiterhin von einigen Autoren als sequentielles Prozessieren in bestimmten, hierarchisch aufgebauten Abfolgen, an denen spezifische Hirnregionen beteiligt sind, verstanden (z.B. Posner \& Petersen, 1990). Andere Autoren legen weniger Wert auf diesen hierarchischen Aspekt der Informationsverarbeitung und konzentrieren sich eher auf die Differenzierung verschiedener funktioneller Einheiten der Informationsverarbeitung (z.B. Mirsky, 1989). Dies ist aus klinisch-neuropsychologischer Sicht interessant, da diese funktionellen Untereinheiten spezifisch beeinträchtigt sein können. Häufig werden folgende funktionelle Aspekte der Informationsverarbeitung unterschieden: (1) selektive (fokussierte) Aufmerksamkeit, d.h. die Fähigkeit, sich aus einer Vielzahl von Reizen auf bestimmte Reize gezielt zu konzentrieren und diese bewußt zu verarbeiten, (2) Vigilanz (engl. sustained attention), d.h. die Fähigkeit, die mentale Aktivität über eine bestimmte Zeit gezielt aufrechtzuerhalten, (3) geteilte Aufmerksamkeit, d.h. die Fähigkeit, mehrere Reize simultan zu verarbeiten oder mehrere kognitive Operationen gleichzeitig durchzuführen, und (4) Wechsel des Aufmerksamkeitsfokus, d.h. die Fähigkeit, den Fokus der mentalen Aktivität von einem Reiz auf einen anderen zu wechseln. Als neuroanatomische Korrelate verschiedenster funktioneller Einheiten der Informationsverarbeitung (Aspekte der Aufmerksamkeitsfunktionen, working memory) werden prefrontale und parietale Strukturen diskutiert (für einen Überblick, s. Cabeza \& Nyberg, 2000).

\subsubsection{Empirische Befunde zu einzelnen klinischen Meßinstrumenten der Informati- onsverarbeitung}

In der klinischen Neuropsychologie werden verschiedene Verfahren eingesetzt, um differentielle Leistungen in der Informationsverarbeitung zu erfassen. Neben einfachen PaperPencil-Tests werden mittlerweile auch computergestützte Tests durchgeführt, die den Vorteil einer automatischen quantitativen und qualitativen Analyse liefern. Die Testbatterie zur Aufmerksamkeitsprüfung (TAP) ist ein Beispiel für solche computergestützten Testverfahren (Zimmermann \& Fimm, 1993). Mithilfe verschiedener Untertests können unterschiedliche Leistungsaspekte der Informationsverarbeitung erfaßt werden:

Geteilte Aufmerksamkeit: Dieser Untertest überpüft in einem Dual-Task-Paradigma die Fähigkeit, auf simultan dargebotene Reize, die aus zwei Modalitäten stammen (Hören und Sehen), zu reagieren. Die klinische Relevanz von Leistungsminderungen bei Dual-TaskAnforderungen konnte sowohl für Patienten mit traumatischen Hirnschädigungen als auch für Patienten mit Frühsymptomen einer Alzheimer-Demenz gezeigt werden (z.B. Baddeley, Baddeley, Bucks \& Wilcock, 2001; Perry \& Hodges, 1999; Zomeren \& Burg, 1985). 
Reaktionswechsel: Selektive Aufmerksamkeitsleistungen setzen neben der Fähigkeit zur Fokusierung und Aufrechterhaltung auch die Fähigkeit voraus, den Aufmerksamkeitsfokus zu wechseln (vgl. Posner, Friedrich, Walker \& Rafel, 1984). Generell ist die Fähigkeit zum Aufmerksamkeitswechsel eine wesentliche Voraussetzung für die allgemeine mentale Flexibilität (Sohberg \& Mateer, 1987). Klinisch bildet sich die Fähigkeit zum Aufmerksamkeitswechsel auf einem Kontinuum von unkontrolliertem Wechsel (=Ablenkbarkeit) bis zur Unfähigkeit zum Aufmerksamkeitswechsel (=Perseveration) ab.

Wie bereits im vorherigen Abschnitt angesprochen, werden in der modernen klinischen Neuropsychologie Überpüfungen von Arbeitsgedächtnisleistungen als Überprüfungen von Informationsverarbeitungsleistungen verstanden:

Digit Span Forward u. Backward (WMS-R): Während die Forward-Variante des Zahlennachsprechens lediglich passive Elemente der kurzfristigen Einspeicherung verbalen Material überprüft, werden zur Bewältigung der Backward-Variante zusätzlich aktivere Aspekte der Informationsverarbeitung benötigt. Es scheint eine subtile Lateralisierung vorzuliegen: Die Forward-Variante scheint sensitiv für linksseitige, weniger aber für rechtsseitige und diffuse Hirnschädigungen zu sein (Black, 1986), während die Backward-Variante genereller bei Hirnschädigungen, d.h. sowohl bei rechts- als auch linksseitigen und diffusen Schädigungen, Defizite aufdeckt (ebd.; Leininger, Gramling, Farrell, Kreutzer \& Peck, 1990).

Visual Memory Span Forward u. Backward (WMS-R): Dieser Untertest überprüft in seiner Forward-Variante die kurzfristige Behaltensspanne für visuell-räumliche Information. Analog zum Untertest Digit Span werden zur Bewältigung der Backward-Variante aktive mentale Operationen benötigt. Patienten mit traumatischen oder degenerativen Hirnschädigungen zeigen in beiden Formen dieses Untertests unterdurchschnittliche Leistungen (Canavan et al., 1989; Sullivan, Corkin \& Growdon, 1986).

Arbeitsgedächtnis (TAP): Dieser Untertest verlangt eine kontinuierliche Kontrolle des Informationsflusses durch den Kurzzeitspeicher, da der aktuell präsentierte Reiz mit zuvor präsentierten verglichen werden muß. Die Bewältigung der Aufgabe wird bereits von gesunden Probanden als schwer empfunden.

Darüber hinaus ist im Rahmen klinischer Fragestellungen die Rate der mentalen Aktivität interessant, da bei vielen neuropsychologischen Patienten eine Verlangsamung z.B. der Psychomotorik prominent ist und natürlich einen Einfluß auf die o.g. funktionellen Einheiten 
der Informationsverarbeitung hat. Die psychomotorische Geschwindigkeit wird in vielen Studien mithilfe des Trail Making Tests (TMT) erfaßt (Reitan, 1992), während z.B. der WMS-RUntertest Mental Control allgemeinere Aspekte der mentalen Aktivität überprüft.

Trail Making Test Form A und B: Form A dieses Tests gilt als Messung der einfachen psychomotrischen und visuellen Suchgeschwindigkeit. Form B verlangt zusätzlich die Fähigkeit, zwischen Zahlen und Symbolen zu wechseln, und überprüft somit neben der visuomotorischen Suchgeschwindigkeit auch die Fähigkeit, den Aufmerksamkeitsfokus zu wechslen (s.o.). Beide Formen gelten als sensitiv für verschiedenste Formen der Hirnschädigung, sowohl traumatischer (vgl. Leininger et al., 1990) als auch progredient-dementieller Ätiologie (vgl. Perry \& Hodges, 1999).

Mental Control (WMS-R): Der Untertest besteht aus drei Aufgaben: Während die dritte Aufgabe einen leicht erhöhten Schwierigkeitsgrad hat (Addition in Dreier-Schritten, also "1,4,7.."), sind die zwei ersten Aufgaben (Aufsagen des Alphabets; Rückwärtszählen von 20 auf 1) so leicht und überlernt, daß sie zwischen Personen mit leichter bis mittelschwerer Hirnschädigung und gesunden Kontrollpersonen kaum differenzieren. Allerdings scheint der Untertest die zunehmende kognitive Desorientiertheit von Alzheimer-Patienten zu reflektieren (vgl. Botwinick, Storandt \& Berg, 1986).

\subsection{KeRnSPINTOMOGRAPHISChe DiAgNOSE VON GEWEbSSCHÄDIGUN- GEN DES GEHIRNS}

Eine kernspintomographische Untersuchung (=Magnetresonanztomographie, MRT) umfaßt folgende Schritte: Der Patient wird in ein Magnetfeld gebracht, eine elektromagnetische Welle wird eingestrahlt und wieder ausgeschaltet. Das daraufhin vom Patienten ausgesendete Signal wird aufgefangen und dient als Grundlage der Bildkonstruktion. Die Magnetresonanztomographie beruht auf den magnetischen Eigenschaften der Wasserstoffatomkerne im zu untersuchenden Organismus. Einem Magnetfeld ausgesetzt, richten sich die Wasserstoffatomkerne im Körper parallel zu den Feldlinien aus. Dabei werden durch eingestrahlte Hochfrequenzimpulse bestimmte Energiezustände der Atomkerne bewirkt. Die nach Abschalten bzw. bei der Relaxation der Atome in ihre Ausgangslage wieder freiwerdende eingestrahlte Energie bewirkt die eigentliche Bildgebung. Im MRT-Bild entsprechen unterschiedliche Signalintensitäten unterschiedlichen Protonendichten und somit unterschiedlichen Gewebedichten. Auf die präzise Darstellung der physikalischen Hintergründe und technischen Details wird an dieser Stelle verzichtet. Die für die neuroradiologische Interpretation cranialer 
Kernspintomogramme wichtigen Aufnahmeparameter sollen jedoch kurz erläutert werden. Diese Parameter verändern die Darstellung von Geweben, so daß in Abhängigkeit zur Fragestellung bestimmte Parametereinstellungen günstiger sind als andere: Die Wahl der eingestrahlten elektromagnetischen Welle, des sogenannten (Im-)Pulses, sowie die Wahl bestimmter Zeitparameter, d.h. z.B. der Zeit zwischen den einzelnen Pulsen, führen zu unterschiedlichen Gewichtungen, d.h. zu unterschiedlichen Signalgebungen ein- und desselben Gewebes. MRTs können $\mathrm{T}_{1^{-}}, \mathrm{T}_{2^{-}}$oder Protonen-Dichte (=proton-density)-gewichtet sein. Als Faustregel gilt: Wenn Liquor weiß erscheint, liegt ein $\mathrm{T}_{2}$-gewichtetes Bild vor. Wenn der Liquor hingegen dunkler als das Hirngewebe erscheint, ist das Bild $\mathrm{T}_{1}$ - oder Protonen-Dichte-gewichtet. Während bei einer $\mathrm{T}_{1}$-Gewichtung die graue Substanz des Gehirns auch tatsächlich grauer erscheint als die weiße Substanz, erscheint in einem Protonen-Dichte-gewichteten Bild die graue Substanz heller als die weiße Substanz, weil erstere aufgrund ihres höheren Wasseranteils, also ihrer höheren Protonendichte, ein stärkeres Signal liefert.

Lange Zeit galt die $\mathrm{T}_{2}$-gewichtete Spin-Echo-Pulssequenz als "gold standard" bei der kernspintomographischen Diagnose von pathologischen Veränderungen des Hirngewebes z.B. nach cerebrovaskulären Vorfällen oder bei Verdacht auf Tumorbildung (DeCoene, Hajnal \& Gatehouse, 1992). Es zeigte sich jedoch, daß subtile Parenchymveränderungen vor allem in bestimmten Bereichen des Gehirns durch diese Sequenzen nicht erfaßt werden. Gründe dafür sind Artefakte, die von der Cerebrospinalflüssigkeit ausgehen, die auf $\mathrm{T}_{2}$-gewichteten Aufnahmen die höchste Signalintensität hat. Das führt dazu, daß im Bereich um die Seitenventrikel (=periventrikulärer Bereich), aber auch in Übergangsbereichen zwischen weißer und grauer Substanz Läsionen nicht oder nicht eindeutig entdeckt werden (ebd.). Durch die Etablierung einer neuen Pulssequenz, der Inversion Recovery, ist es möglich, diese Artefakte auszuschalten, da hierbei die Signalgebung der Cerebrospinalflüssigkeit vollständig neutralisiert werden kann. Aufnahmen mit der FLAIR-(=Fluid Attenuation Inversion Recovery)-Sequenz bieten also höhere Kontraste für sehr kleine Läsionen in der weißen Substanz und der subcorticalen grauen Substanz.

Quantitative Auswertungen von dreidimensionalen kernspintomographischen Datensätzen erlauben die voxelbasierte Volumenbestimmung des Gesamtgehirns, aber auch von Teilstrukturen, den sogenannten Regions of Interest (ROIs). Hierzu müssen die digitalisierten Daten auf einen Computer überspielt und mit einer entsprechender Software bearbeitet werden. 3-D-Datensätze basieren meist auf $\mathrm{T}_{1}$-gewichteten Aufnahmen. 


\subsection{White MATTER HYPERINTENSITÄTEN UND LAKUnÄRE INFARZIE- RUng Bei Patienten mit Koronarer Herzkrankheit}

\subsubsection{White MATTER HYPERINTENSITÄTEN}

Die weiße Substanz bezeichnet die häufig zu Faserbündeln (=fasciculi) vereinten Axone der im Neocortex und in den subcorticalen Kernen liegenden Neurone und nimmt im adulten Gehirn nahezu die Hälfte des Gesamtvolumens ein. Sie bildet das Netzwerk, in dem Informationen zwischen verschiedenen Hirnarealen ausgetauscht werden. Drei Haupt-Leitungsbahnen können unterschieden werden: Projektionen sensorischer und motorischer Informationen zum und vom Neocortex, kommissurale Faserstränge, vorrangig das Corpus callosum, die beide Hemisphären miteinander verbinden, und schließlich Assoziationsbahnen, die die Kommunikation corticaler Areale untereinander ermöglichen. Bei letzteren wird zwischen sogenannten U-Fasern, die benachbarte Gyri verbinden, und langen Assoziationsfasern, die entferntere intrahemisphärische Areale verknüpfen, unterschieden (Kolb \& Wishaw, 1993). Die weiße Substanz wird metabolisch hauptsächlich über lange penetrierende Arterien versorgt, die sich von basal liegenden, großen Hauptarterien abzweigen. Lediglich die Versorgung der direkt unter dem Neocortex liegenden U-Fasern erfolgt über kurze Arterien, die von Adern an der Cortexoberfläche stammen. Die Art der Versorgung hat entscheidende pathophysiologische Konsequenzen: Die langen penetrierenden Markarterien sind für arteriosklerotische Veränderungen wie z.B. Lipohyalinose prädisponiert, was häufig zu mikroangiopathischen Ischämien im Versorgungsbereich führt ("small vessel disease") (Chimowitz, Awad \& Furlan, 1989).

Eine weiteres physiologisches Merkmal der weißen Substanz ist ebenfalls von entscheidender Bedeutung: Die axonale Reizleitungsgeschwindigkeit wird durch die Myelinisierung der Axone optimiert. Saltatorische Impulsausbreitung zwischen den von der Myelinschicht gebildeten Ranvierschen Schnürringen erlaubt eine sehr viel schnellere Reizweiterleitung in myelinisierten als in nicht-myeliniserten Fasern. Eine Zerstörung der Myelinschicht z.B. durch entzündliche Prozesse im Rahmen der Multiplen Sklerose führt also bereits zu Leitungsstörungen und damit verbundenen klinischen Symptomen, obwohl die Axone nicht zerstört sind. In diesem Falle, aber auch bei anderen Erkrankungen, die die weiße Substanz betreffen, kommt es zu klinischen Symptomen, ohne daß die entsprechenden Nervenzellen in der corticalen und subcorticalen grauen Substanz beschädigt sind. Symptome, die auf Zerstörung der Leitungsbahnen zurückgehen, sind häufig nicht von durch Läsionen der grauen Substanz entstandenen Symptomen zu unterscheiden. Kognitive, emotionale und verhaltenssteu- 
ernde Funktionen basieren auf der komplexen Verschaltung verschiedener Neuronenverbände. Damit liegt die Bedeutung der das Netzwerk unterhaltenden weißen Substanz auf der Hand (Filley, 1998).

Kognitive und emotionale Defizite nach Schädigungen der weißen Substanz sind bereits lange bekannt. Im Jahr 1894 beschrieb Otto Binswanger den Fall eines Mannes mit progressivem kognitiven Verfall bezüglich Sprach- und Gedächtnisfunktionen sowie psychopathologischen und neurologischen Auffälligkeiten. Die Autopsie des Gehirns zeigte eine cerebrale Atrophie der weißen Substanz und lakunäre Infarzierung der subcorticalen grauen Substanz bei intracranialer Arteriosklerose (Binswanger, 1894). Einige Jahre später bezog sich Alzheimer auf diese Fallbeschreibung und faßte die beschriebenen Veränderungen unter "Binswanger's Encephalitis subcorticalis" zusammen (Alzheimer, 1902). Jahrzehnte später führte O1zewski den Begriff "subcortical arteriosclerotic encephalopathy" ein (Olzewski, 1962). Die Literaturübersicht der Autoren Babikian und Ropper zeigte, daß bis zum damaligen Jahr 1987 lediglich 50 pathologisch gestützte Fälle dieser Erkrankung beschrieben worden waren (Babikian \& Ropper, 1987). Morbus Binswanger bzw. die subcorticale arteriosklerotische Encephalopathie wurde damit zu einer "obscure entity" (Pantoni \& Garcia, 1995). Durch die neuen bildgebenden Verfahren kam es zu einer Renaissance, da nun prämortem Auffälligkeiten in der weißen Substanz diagnostiziert werden konnten. Gleichzeitig kristallisierte sich jedoch heraus, daß die kernspintomographisch identifizierbaren Signalanhebungen weder in ihrer klinischen Relevanz noch in ihrem histopathologischen Korrelat eindeutig waren. Hachinski führte 1987 den Begriff "leuko-araiosis" für computertomographisch identifizierbare "white matter lucencies" ein (Hachinski, Potter \& Merskey, 1987). Der Begriff "white matter hyperintensities" (WMH) ist als Deskription der Signalanhebungen innerhalb der weißen Substanz in kernspintomographischen Aufnahmen jedoch noch geeigneter und mittlerweile auch geläufiger. Auf Begriffe wie Morbus Binswanger oder subcorticale arteriosklerotische Encephalopathie sollte in Untersuchungen mit gesunden und neurologisch unauffälligen Probanden verzichtet werden. Beide Begriffe umfassen die Diagnose einer degenerativen dementiellen Erkrankung mit klinisch relevanten kognitiven und neurologischen Symptomen (Fazekas, Schmidt, Kleinert et al. 1998).

\subsubsection{Auftretenshäufigkeit von White Matter Hyperintensitäten und assoziierte Risikofaktoren}

Es ist nicht möglich, eine exakte Prävalenz für das Auftreten von White Matter Hyperintensitäten anzugeben. Die Studien unterscheiden sich in einer Vielzahl wesentlicher Para- 
meter, so daß die jeweiligen Prävalenzraten nur schwer zu vergleichen sind. Alter und medizinischer Status der untersuchten Probanden sind ebenso entscheidend wie die bereits beschriebenen Aufnahmeparameter der kernspintomographischen Untersuchung. Die Prävalenzraten für $W M H$ in verschiedenen Studien schwanken zwischen $20 \%$ und 100\%. An diesen beiden Extremwerten wird die Bedeutung der Stichprobenzusammensetzung deutlich. In der Studie, in der lediglich 20\% der Probanden $W M H$ aufwiesen, wurden gesunde und junge, d.h. im Mittel 38 Jahre alte Probanden untersucht (Schmidt, Fazekas, Offenbacher, Dusleag \& Lechner, 1991). Hingegen handelte es sich in der Studie, in der alle untersuchten Probanden WMH aufwiesen, um im Mittel 66 Jahre alte Probanden mit Bluthochdruck, Diabetes mellitus und kleineren cerebrovaskulären Vorfällen in der Anamnese (Junqué et al., 1990). Höheres Lebensalter und vaskuläre Risikofaktoren, vor allem Bluthochdruck, sowie das Vorliegen einer KHK erhöhen die Auftretenshäufigkeit von im Kernspintomogramm sichtbaren Hyperintensitäten in der weißen Substanz (Fazekas et al., 1988; Hébert et al., 2000; Lechner et al., 1988; Longstreth et al., 1998; Manolio et al., 1994; Özeren et al., 1998). Lindgren und Mitarbeiter konnten in einer logistischen Regressionsanalyse zeigen, daß Lebensalter und Herzerkrankungen unabhängige Risikofaktoren darstellen (Lindgren et al., 1994). In zwei Followup-Studien wurde das Vorliegen von Bluthochdruck zum ersten Erhebungszeitpunkt als wichtigster Prädiktor für die Progression der $W M H$ nach zwei bzw. drei Jahren identifiziert (Schmidt, Fazekas, Kapeller, Schmidt \& Hartung, 1999; Veldink, Scheltens, Jonker \& Launer, 1998).

Eine weitere Varianzquelle der Prävalenzraten stellen die unterschiedlichen visuellen Rating-Systeme dar, mit denen die Signalanhebungen im Kernspintomogramm beurteilt werden. Im Auftrag der European Task Force on Age-Related White Matter Changes erstellten Scheltens und Kollegen einen Überblicksartikel, in dem verschiedene Rating-Skalen bezüglich Praktikabilität, Reliablität und Validität verglichen wurden (Scheltens et al., 1998). Einen befriedigenden Kompromiß zwischen Anforderungen verschiedener Studiendesigns (epidemiologische vs. klinisch orientierte Studien) stellt die Skala von Fazekas und Mitarbeitern dar (Fazekas, Chawluk, Alavi, Hurtig \& Zimmermann, 1987), die zwischen periventrikulären Hyperintensitäten (PVH) und Hyperintensitäten in der deep white matter (DWHM) unterscheidet und diese in jeweils vier Schweregrade einteilt (DWMH: $0=$ absent; $1=$ =punctate foci; $2=$ early confluence of foci; $3=$ large confluent ares $)(P V H: 0=$ absent; $1=$ caps and rims/pencilthin lining; 2=halo; 3=irregular PVH extending in the DWM). Wegen ihrer hohen Praktikabilität wurde die Fazekas-Skala seit 1987 in zahlreichen Studien eingesetzt. Mittlerweile wurde eine histopathologische Validierung der Skala vorgenommen (Fazekas et al., 1987; Fazekas et 
al., 1991; Fazekas et al., 1993). Die Autoren schlagen eine Modifikation auf Basis der histopathologischen Befunde vor, die von einer Unterscheidung zwischen deep white matter Hyperintensitäten und periventrikulären Hyperintensitäten absieht sowie leichtgradigen periventrikulären Veränderungen im Sinne von "caps" und "halos" keinen pathologischen Wert beimißt.

\subsubsection{Morphologische Korrelate der im Kernspintomogramm sichtbaren White Mat- ter Hyperintensitäten}

Hyperintensitäten, also Signalanhebungen in $T_{2}$-gewichteten Aufnahmen wie auch in FLAIR-Sequenz-Aufnahmen, zeigen eine erhöhte Protonendichte im betreffenden Gewebe an, d.h. der Wasseranteil ist hier höher als im umliegenden Gewebe. Pathophysiologische Prozesse, die zu einem erhöhten Wasseranteil führen können, sind Ödeme (entzündlich, cytotoxisch oder vasogen), Vakuolation (Entwicklung flüssigkeitsgefüllter Räume im Hirnparenchym), Infarzierung (akut: Ödem, chronisch: Ersetzen des nekrotischen Gewebes durch flüssigkeitsgefüllte Räume) oder Gliosis (Ersetzen myelinisierter Axone in einem astrocytischen Prozeß, der mit erhöhter Wasserdichte einhergeht) (Chimowitz et al., 1989). Eine Hyperintensität im Kernspintomogramm ist nicht spezifisch, läßt also nicht erkennen, welche der genannten Gewebsschädigungen vorliegt und welcher pathophysiologische Prozeß dementsprechend zugrundeliegt (Fazekas et al., 1998).

Histopathologische Untersuchungen versuchen die Frage $\mathrm{zu}$ beantworten, welche Gewebsveränderungen in von $W M H$ betroffenen Arealen makro- und mikroskopisch zu identifizieren sind. In der Regel werden dazu die in Formalin fixierten Gehirne kurz zuvor verstorbener Patienten zunächst kernspintomographisch und dann histopathologisch untersucht. Ein anderes Vorgehen ist der Vergleich von prämortem erhobenen kernspintomographischen Befunden mit histopathologischen Ergebnissen der Autopsie. Dieses Vorgehen wird jedoch kritisch gesehen, da die terminalen Abläufe eventuell zu Veränderungen im Gehirn führen, die auf dem Kernspintomogramm natürlich nicht zu sehen sind (Munoz, Hastak, Harper, Lee \& Hachinski, 1993).

Zwei Befunde sind wiederholt repliziert worden: Gewebsareale, die $W M H$ zeigen, korrespondieren zum einen histopathologisch mit erweiterten perivaskulären Räumen (sog. Virchow-Robin-Räume), die sich mit extracellulärem Wasser füllen und somit zu einer Signalanhebung führen. Zum anderen läßt sich in WMH-Arealen Demyelinisierung mit reaktiver Gliosis nachweisen, ein Prozeß, der ebenso zu einer erhöhten Wasserdichte und entsprechend zu 
einem hyperintensiven Signal führt. In einer ausführlichen Studie kommen Fazekas und Mitarbeiter (Fazekas et al., 1993) zu folgenden Schlußfolgerungen: Punktuelle, beginnend konfluierende und deutlich konfluierende Hyperintensitäten in der deep white matter korrespondieren mit im Schweregrad ansteigenden ischämisch bedingten Gewebsschädigungen. Diese Gewebsschädigungen rangieren von leichten perivaskulären Veränderungen bis hin zu großflächigen Bereichen mit Faserverlust, Demyelinisierung, multiplen kleinen Kavernen und begleitender Arteriosklerose. Auch irreguläre periventrikuläre Signalintensitäten fallen in diese letzte Kategorie. Periventrikuläre "caps" und "halos" hingegen werden als nicht ischämisch bedingte Gewebschädigungen bewertet, die eher vor dem Hintergrund prädisponierender anatomischer Besonderheiten Resultat einer chronisch erhöhten perivaskulären Flüssigkeitskonzentration sind. Die Beurteilung konfluierender $D W M H$ und irregulärer $P V H$ als eindeutig pathologische Veränderungen mit Demyeliniserung und reaktiver Gliosis wird auch von anderen Autoren geteilt (Munoz et al., 1993; Scarpelli et al., 1994). Es besteht jedoch Uneinigkeit darüber, wie Befunde zu erweiterten Virchow-Robin-Räumen in periventrikulären hyperintensiven Arealen (="caps" und "halos") zu bewerten sind. Während in einigen Studien erweiterte Virchow-Robin-Räume als Anfangspunkt eines pathologischen Kontinuums und Prozesses beurteilt werden, an dessen Ende dann Gliosis steht (Awad, Johnsons, Spetzler \& Hodak, 1986), interpretieren andere Autoren wie z.B. Scarpelli erweiterte Virchow-Robin-Räume als nichtpathologische Verursachung von periventrikulären "caps" und "halos", die auf anatomische Gegebenheiten des Gewebes zurückzuführen sind (Scarpelli et al., 1994). Easton kommt ebenfalls zu dem Schluß, daß periventrikuläre "caps" und "halos" nicht ischämischpathologischen Ursprungs sind. Irreguläre $P V H$ sind seiner Meinung nach fälschlicherweise mit periventrikulären Veränderungen wie "caps" und "halos", gleichgesetzt worden, obwohl sie eher $D W M H$ sind, die sich bis an die Hörner der Seitenventrikel ziehen (Easton, 1997).

\subsubsection{Klinische Relevanz von White Matter Hyperintensitäten}

Während Veränderungen der White Matter, die computertomographisch sichtbar sind, eindeutig mit fokalen neurologischen Zeichen, Schwere der Alzheimer-Demenz und -bei ansonsten unauffälligen Probanden- mit leichteren kognitiven Defiziten korreliert sind (Steingart et al., 1987), ist die Befundlage zu kernspintomographisch sichtbaren Läsionen nicht eindeutig. Das ist auf die geringere Sensitivität der Computertomographie zurückzuführen, die erst dann positive Befunde zeigt, wenn bereits ein substantieller Krankheitswert vorliegt (Easton, 1997). Zudem werden häufig Skalen zur Erhebung kognitiver Funktionen eingesetzt, die eher globale Beeinträchtigungen objektivieren, z.B. die "Mini-Mental-State- 
Examination" (Folstein, Folstein \& McHugh, 1975). Diese Instrumente sind nicht geeignet, subtile neuropsychologische Defizite zu erfassen. Zusammenhänge zwischen subtilen kognitiven Defiziten und leichtgradigen, aber im sensitiven Kernspintomogramm bereits sichtbaren Veränderungen der White Matter bleiben in diesen Studien somit häufig unerkannt. GunningDixon und Raz werteten 23 Studien zum Zusammenhang zwischen $W M H$ und kognitiver Leistungsfähigkeit meta-analytisch aus und kamen zu folgendem Ergebnis: Höhere $W M H$ Scores waren assoziiert mit schlechterer Performanz in Tests, die die globale kognitive Leistungsfähigkeit, die Verarbeitungsgeschwindigkeit, die Einspeicher- und Abrufleistung sowie exekutive Funktionen überprüfen. Weitergehende varianzanalytische Auswertungen zeigten die stärksten Zusammenhänge zwischen $W M H$ und Verarbeitungsgeschwindigkeit bzw. exekutiven Funktionen an (Gunning-Dixon \& Raz, 2000). In diese Meta-Analyse gingen lediglich Untersuchungen mit gesunden, neurologisch und kognitiv unauffälligen Probanden ein. Studien mit dementen Patienten oder Patienten mit cerebrovaskulären Risikofaktoren zeigen jedoch ähnliche Ergebnisse, häufig korreliert die Schwere der $W M H$ auch mit sog. neurologischen soft signs (z.B. Primitivreflexe, Gangunsicherheit) (Fukuda, Kobayashi, Okada \& Tsunematsu, 1990; Junqué et al., 1990).

Neben der Frage, welchen Einfluß das Vorliegen von $W M H$ auf die aktuelle kognitive Leistungsfähigkeit hat, interessiert der prognostische Wert solcher Befunde. Follow-upStudien untersuchen, ob Probanden mit $W M H$ zur ersten Erhebung sich zur zweiten Erhebung von Probanden ohne WMH in neuropsychologischen Tests unterscheiden. Austrom und Mitarbeiter fanden keine Leistungsunterschiede zwischen Probanden, die 18 Monate zuvor WMH aufgewiesen hatten, und damals unauffälligen Probanden (Austrom, Thompson \& Hendric, 1990). Schmidt und Kollegen fanden zwar eine Progredienz der $W M H$ nach drei Jahren, vor allem bei Probanden mit bereits konfluierenden $W M H$ zum ersten Erhebungszeitpunkt, konnten jedoch keinen Zusammenhang mit einer Verschlechterung kognitiver Leistungen zeigen (Schmidt et al., 1999). Anders hingegen eine Studie, in der Probanden untersucht wurden, die zum ersten Erhebungszeitpunkt bereits ein "mild cognitive impairment" (MCI) aufwiesen: Nach zwei Jahren hatten acht der 27 Studienteilnehmer eine Demenz entwickelt. Diese acht hatten sich zur Baseline-Erhebung in der Schwere der WMH und der ebenfalls erhobenen Temporallappenatrophie von Probanden ohne MCI unterschieden (Wolf, Ecke, Bettin, Dietrich \& Gertz, 2000).

Pathophysiologische Untersuchungen (Positron-Emissions-Tomographie "PET" und Single-Photon-Emissions-Computertomographie "SPECT"), die den regionalen cerebralen 
Blutfluß bzw. die Glucoseverstoffwechslung abbilden, zeigen, daß das Vorliegen von $W M H$ per se nicht zu einer Hypoperfusion bzw. einem Hypometabolismus führt. Lediglich bei Probanden, die zusätzlich zu den kernspintomographischen Auffälligkeiten auch deutlich kognitiv beeinträchtigt waren, zeichneten sich pathophysiologische Befunde ab (Sabri et al., 1999; Yao et al., 1992).

\subsubsection{LAKUNÄRE INFARZIERUNG}

Neben den beschriebenen $W M H$ sind lakunäre Infarkte ebenfalls ein häufiger (Zufalls-) Befund kernspintomographischer Untersuchungen (Longstreth et al., 1998). Lakunen sind definiert als multiple, umschriebene, subcortical gelegene Defekte mit einem Durchmesser von 2-10 mm. Sie entstehen meist auf der Basis von autochthonen Thrombosen kleiner, penetrierender Markarterien und treten bevorzugt in den Basalganglien, der Capsula interna und im Hirnstamm auf, seltener auch in thalamischen Kernen (Lezak, 1995; Poeck, 1994). Mit lakunärer Infarzierung assoziierte Risikofaktoren sind neben höherem Lebensalter und Hypertonie in etwas weniger deutlichen Zusammenhängen auch weitere vaskuläre Risikofaktoren wie Adipositas, Nikotinabusus und Diabetes mellitus (Tanizaki et al., 2000). Kardiale Erkrankungen stellen ein Risiko im Sinne einer potentiellen Emboliequelle dar: Aus den Herzgefäßen können sich Emboli lösen, in den cerebralen Blutkreislauf geraten und in bereits stenosierten kleineren Hirngefäßen einen Verschluß bilden (Mäntyla et al., 1999). Die Übereinstimmung bezüglich assoziierter Risikofaktoren und die hohe Komorbiditätsrate von lakunärer Infarzierung und $W M H$ lassen vermuten, daß beide Befunde Epiphänomene eines gemeinsamen mikroangiopathischen Prozesses sind (Hirono, Yasuda, Tanimukai, Kitagaki \& Mori, 2000; Mäntyla et al., 1999; Sabri et al., 1999).

Die Frage nach der klinischen Relevanz lakunärer Infarzierungen ist nicht eindeutig zu beantworten. Makroinfarkte in den oben genannten Hirnarealen führen zu typischen neurologischen und neuropsychologischen Ausfällen (Boiten \& Lodder, 1992). Anders hingegen die lakunäre Infarzierung dieser Areale: Sie stellt häufig einen Zufallsbefund im Rahmen von aus anderen Gründen durchgeführten Kernspintomographien dar, d.h. sie ist subklinisch verlaufen. In einer großangelegten epidemiologischen Studie mit Probanden höheren Lebensalters konnten bei 23\% der Teilnehmer lakunäre Infarkte im Kernspintomogramm identifiziert werden, von denen $89 \%$ subklinisch verlaufen waren, d.h. es hatte anamnestisch keine Hinweise auf eine transiente ischämische Attacke (TIA) oder einen Hirninfarkt gegeben (Longstreth et al., 1998). Interessanterweise zeigte diese Studie jedoch trotz subklinischer Verläufe schlech- 
tere kognitive Leistungen und eine erhöhte Prävalenzrate subtiler neurologischer Zeichen bei Probanden mit lakunärer Infarzierung im Vergleich zu Probanden ohne diese Befunde. Auch andere Studien konnten kognitive Leistungsunterschiede objektivieren, zumeist in neuropsychologischen Tests, die im weitesten Sinne frontale Funktionen wie mentale Kontrolle, Arbeitsgedächtnis und schlußfolgerndes Denken überprüfen (Corbett, Benett \& Kos, 1994; Kramer, Reed, Mungas, Weiner \& Chui, 2002). Die Beeinträchtigung frontaler Funktionen durch lakunäre Infarzierung subcorticaler Kernstrukturen kann interpretiert werden als Diskonnektion fronto-subcorticaler Verschaltungen, die in der Literatur bereits seit langem beschrieben wird und zumindest im Falle degenerativer subcorticaler Erkrankungen wie Morbus Parkinson empirisch bestätigt wurde (Mega \& Cummings, 1994). Interessant sind in diesem Zusammenhang Ergebnisse zum impliziten Lernen motorischer Sequenzen. Implizitmotorisches Lernen wird häufig mittels der Seriellen Reaktionszeitaufgabe (SRTT) überprüft, die 1987 von Nissen \& Bullemer eingeführt und seitdem vielfältig modifiziert wurde. Patienten mit degenerativen subcorticalen Erkrankungen wie z.B. Morbus Parkinson zeigten sich beeinträchtigt beim impliziten Lernen einer motorischen Sequenz (Pascual-Leone et al., 1993), während bei Patienten mit vaskulären Infarkten keine Defizite festgestellt werden konnten (Exner, Koschack \& Irle, 2002, in Druck). Ob Probanden mit lakunären Infarzierungen subcorticaler Kerne und Veränderungen der weißen Substanz im impliziten Lernen beeinträchtigt sind, ist bisher noch nicht untersucht worden.

Zusammenfassend deuten sich folgende Zusammenhänge an: Die Prävalenz von $W M H$ unterliegt einem altersabhängigen Prozeß. WMH per se führen bei ansonsten gesunden Probanden zu subtilen kognitiven Defiziten, die sich als Gruppenmittelwertsunterschiede abbilden lassen. Die klinische und prognostische Relevanz von $W M H$ scheint sich zu erhöhen, wenn weitere Anzeichen mikroangiopathischer Prozesse hinzukommen, z.B. vaskuläre Risikofaktoren, vor allem Hypertension, und eine lakunäre Infarzierung der subcorticalen grauen Substanz vorliegt. Die neuropsychologischen Defizite sind heterogen und betreffen Bereiche des Gedächtnisses und der Informationsverarbeitung, aber auch exekutive Funktionen wie z.B. schlußfolgerndes Denken.

\subsection{Pathologische Veränderungen des Temporallappens bei Patienten mit Koronarer Herzkrankheit}

Die Temporalregion ist lediglich morphologisch definiert; sie umfaßt den primären und sekundären auditorischen Cortex, Teile des sekundären visuellen Cortex, den tertiären senso- 
rischen Cortex sowie den limbischen Cortex. Eine detailliertere Unterteilung des Temporallappens gestaltet sich schwierig. Es gibt keine exakte Übereinstimmung morphologischer und cytoarchitektonischer Areale; werden zusätzlich noch funktionelle Daten, d.h. Läsionsstudien und Ergebnisse bildgebender Verfahren hinzugenommen, steigert sich die Komplexität um ein Vielfaches (Roland \& Zilles, 1998). Die cytoarchitektonische Einteilung trennt den neocorticalen Temporallappen mit der für den Isocortex typischen 6-Schicht-Struktur vom limbischen Temporallappen mit der für den Allocortex typischen Cytoarchitektur mit drei bis maximal fünf differenzierbaren Schichten.

\subsubsection{NEUROPATHOLOGISCHE VERÄNDERUNGEN DES TEMPORALLAPPENS}

Alois Alzheimer beschrieb 1907 erstmals "merkwürdige Veränderungen der Neurofibrillen" als Ursache für die präsenile Demenz (Alzheimer, 1907). Als er entdeckte, daß auch ältere demente Patienten diese neurofibrilläre Degenerationen aufwiesen, verwarf er diese Hypothese wieder (Alzheimer, 1911). Auch eine an anderer Stelle geäußerte Vermutung, eine weitere Auffälligkeit im Gehirn dementer Patienten, die corticalen Plaques (=herdförmige, extrazelluläre Ablagerungen), sei ursächlich für die Demenz, konnte er richtigstellen: Er zeigte, daß sich auch in Gehirnen gesunder älterer Menschen diese ursprünglich als pathognomonisch angenommenen Plaques gehäuft nachweisen ließen. Neuere neuropathologische Arbeiten der letzten Jahre zeigen, daß statt der quantitativen Plaquebeladung des Cortex die Abnahme der corticalen Synapsendichte (Lassmann, Fischer \& Jellinger, 1993) sowie die Ausbreitung der neurofibrillären Degeneration die am engsten mit der Alzheimer-Demenz assoziierten morphologischen Korrelate sind (Braak et al., 1999).

Als neurofibrilläre Degeneration, engl. "neurofibrillary tangles" (NFT), werden Veränderungen des Cytoskeletts von Neuronen beschrieben. In diesen Neuronen finden sich paarig miteinander verschraubte Filamente. Hauptbestandteil dieser intrazellulären helikalen Filamente sind Neurofilamente sowie verschiedene Mikrotubuli-assoziierte Proteine einschließlich einer hyperphosporylierten Form des sogenannten tau-Proteins. Letzteres wird von vielen Autoren als der initiale pathophysiologische Prozeß gesehen: Sie nehmen an, daß die Formation pathologisch phosphorylierter tau-Proteine in ineffizientem Proteinmetabolismus, synaptischer Fehlfunktionen und beeinträchtigter Signalweiterleitung resultiert. Die Gesamtheit dieser Fehlfunktionen führt letzten Endes zum Tod der betroffenen Zelle (Feany \& Dickson, 1996). 
Da es sich bei der Alzheimer-Demenz um eine primär progrediente Demenz handelt, die in bestimmten klinisch beobachtbaren Schritten verläuft, ist die Frage interessant, ob sich diese Progredienz auch neuropathologisch abbildet. Arbeiten z.B. von Braak und Mitarbeitern weisen eindeutig daraufhin, daß der neuropathologische Prozeß in prädisponierten Hirnarealen beginnt und dann in vorhersagbarer und regelmäßiger Weise auf weitere Gebiete übergeht, bis schließlich im Endstadium fast das gesamte Gehirn betroffen ist (Braack \& Braack, 1991). Als mögliche Ursache für die selektive Vulnerabilität verschiedener Hirnareale und für die schablonenhaft ablaufende Ausbreitung neuropathologischer Veränderungen wird folgendes Phänomen diskutiert: Das sequentielle Auftreten der neurofibrillären Degeneration birgt in beeindruckender Weise die inverse Abfolge der corticalen Myelinisierung während der frühen Entwicklung (Braak \& Braak, 1996; McGeer et al., 1990). Spät myelinisierende corticale Gebiete und Schichten entwickeln sehr viel früher und mit höherer Dichte neurofibrillary tangles als diejenigen Gebiete und Schichten, die zu einem frühen Zeitpunkt der Entwicklung myelinisieren. Es können grob drei Phasen der Ausbreitung neurofibrillärer Veränderungen unterschieden werden (Braak et al., 1999):

Transentorhinale Phase: Zunächst ist nur eine kleine Region im medialen Temporallappen betroffen, die transentorhinale Region. Diese Region fungiert als wichtige Schnittstelle für Projektionen neocorticaler Informationen ins limbische System, d.h. der transentorhinale Cortex projiziert über die Zwischenstation des entorhinalen Cortex zum Hippocampus. Auch wenn bereits weitere Regionen wie der entorhinale Cortex von neuropathologischen Veränderungen betroffen sind, wird die Schwelle zur klinischen Manifestation noch nicht überschritten. D.h., obwohl bereits Alzheimer-typische Veränderungen im Gehirn zu objektivieren sind, muß zu diesem Zeitpunkt noch von einer präklinischen Phase gesprochen werden.

Limbische Phase: Schwerwiegende Veränderungen der transentorhinalen und entorhinalen Region folgen auf die erste präklinische Phase. Die neurofibrilläre Degeneration infiltriert nun auch die hippocampale Formation, spart jedoch isocorticale Gebiete noch aus. Im weiteren Verlauf greift der Prozeß jedoch auch auf höhergeordnete Assoziationsareale des Neocortex über. Durch die Unterbrechung intra-limbischer sowie limbisch-präfrontaler Projektionen können sich in diesem Stadium leichtgradige kognitive Beeinträchtigungen und subtile Persönlichkeitsveränderungen bemerkbar machen.

Neocorticale Phase: Von inferioren temporalen Arealen beginnt nun das superolaterale Übergreifen der neuropathologischen Veränderungen auf multimodale Assoziationsgebiete des Neocortex. Zu diesem Zeitpunkt sind deutliche klinische Manifestationen zu objektivie- 
ren. Im Endstadium der Alzheimer-Demenz sind auch die bis zuletzt verschonten primären sensorischen und motorischen Felder betroffen.

Eine Teilstruktur des Temporallappens, der temporale Pol (entspricht BA 38 ${ }^{1}$ ), ist im Zusammenhang mit der von Braak und Mitarbeitern postulierten sequentiellen Abfolge der Ausbreitung neuropathologischer Veränderungen besonders interessant. Er umfaßt die anteriore Region des Temporallappens von der Spitze an bis zu einem coronalen Level auf Höhe des Limen insulae ${ }^{2}$. Der temporopolare Cortex stellt cytoarchitektonisch einen Übergangsbereich zwischen dem allocorticalen limbischen System und isocorticalen lateralen, ventralen und dorsalen Temporallappenregionen dar (Kim et al., 2000). Er unterhält weitreichende reziproke Projektionen zum temporalen und frontalen Neocortex und zu limbischen sowie subcorticalen Strukturen (Markowitsch, Emmans, Irle, Steicher \& Preilowski, 1985; Morán, Mufson \& Mesulam, 1987). Nach Braak et al. ist anzunehmen, daß in der limbischen Phase der Ausbreitung neuropathologischer Veränderungen (s.o.) der temporale Pol diejenige Struktur ist, die nach der entorhinalen und hippocampalen Region als nächste Struktur von den neuropathologischen Veränderungen betroffen ist. Mit dem temporalen Pol als höhergeordnetem multimodalen Assoziationscortex wäre dann zu einem relativ frühen Zeitpunkt ein Gebiet betroffen, dessen Schädigung kognitive, affektive und verhaltensbezogene Symptome der Alzheimer-Demenz verursachen könnte (Arnold, Hyman \& Hoesen, 1994).

\subsubsection{KERNSPINTOMOGRAPHISCH ERFASSTE VOLUMENREDUKTION VON TEMPORAL- LAPPENSTRUKTUREN}

Nachdem die neuropathologischen Befunde zur Alzheimer-Demenz mittlerweile unumstritten sind, liegt das Forschungsinteresse auf der Entwicklung von Methoden, die eine invivo-Erfassung der pathologischen Veränderungen ermöglichen. Das ist natürlich vor allem deshalb von Interesse, weil die neuropathologischen Ergebnisse daraufhinweisen, daß es bereits $\mathrm{zu}$ morphologischen Veränderungen im medialen Temporallappen kommt, bevor klinisch erste Frühsymptome einer dementiellen Entwicklung diagnostizierbar sind. Die quantitative Auswertung von kernspintomographischen 3D-Datensätzen, d.h. die voxelbasierte Volumenbestimmung einzelner Hirnareale, bietet diese Möglichkeit. Bobinski und Mitarbeiter validierten jüngst die in diesem Falle postmortem vorgenommene kernspintomographische

\footnotetext{
${ }^{1} \mathrm{BA}=$ Areale nach Brodmann (1909)

${ }^{2}$ Bezeichnungen nach Nieuwenhuys, Voogd und van Huijzen (1991)
} 
Volumenmessung medialer Temporallappenstrukturen neuropathologisch und fanden hohe Korrelationen zwischen beiden Volumenmessungen (Bobinski, Leon \& Wegiel, 2000).

Im Mittelpunkt des Interesses bei der quantitativen Auswertung kernspintomographischer 3D-Daten stand die Volumenbestimmung des Hippocampus. $\mathrm{Zu}$ diesem Interessenschwerpunkt führten Kenntnisse aus Läsionsstudien zur herausragenden Rolle des Hippocampus bei der Einspeicherung episodischer und semantischer Gedächtnisinhalte (Kolb \& Wishaw, 1993) und die klinische Beobachtung, daß Gedächtnisdefizite die ersten Frühsymptome im Rahmen der Alzheimer-Demenz sind (McKhann, Drachman, Folstein, Price \& Stadlan, 1984). In zahlreichen Studien konnte eine deutliche Volumenminderung der hippocampalen Formation bei Alzheimer-Patienten gezeigt werden, die sie von nichtdementen Personen gleichen Alters mit hoher Sensitivität diskriminierte. Die Spezifität der Volumenreduktion des Hippocampus bei Alzheimer-Demenz ist hingegen nicht unumstritten: Werden die Hippocampusvolumina von Patientengruppen mit unterschiedlicher Demenzätiologie (Alzheimer vs Lewy-Bodies-Demenz vs vaskuläre Demenz) untereinander verglichen, so hat das Hippocampusvolumen in einigen Studien einen diskriminativen Wert (Barber, Ballard, McKeith, Gholkar \& O'Brien, 2000), in anderen wiederum nicht (Bigler et al., 2000; Laakso et al., 1996).

Angesichts der Deutlichkeit, mit der kernspintomographisch eine Atrophie der hippocampalen Formation nachgewiesen werden kann, stellt sich die Frage, ob neben dem Hippocampus auch weitere Strukturen betroffen sind und ob sich die zeitliche Sequentierung der neuropathologischen Veränderungen auch kernspintomographisch abbilden läßt. Tatsächlich lassen sich neben der Atrophie des Hippocampus auch Volumenminderungen weiterer limbischer Temporallappenstrukuren bei Alzheimer-Patienten nachweisen (Callen, Black, Gao, Caldwell \& Szalai, 2001; Convit et al., 1997; Juottonen, Laakso, Partanen \& Soininen, 1999). Barber und Kollegen konnten neben einer deutlichen Atrophie der hippocampalen Formation auch Volumenminderungen neocorticaler Temporallappenstrukuren wie dem Gyrus temporalis superior, dem Gyrus temporalis medius sowie dem Gyrus temporalis inferior bei Patienten mit Alzheimer-Demenz nachweisen (Barber, McKeith, Ballard, Gholkar \& O’Brien, 2001). Diese Befunde weisen auf die Korrespondenz zwischen der sequentiellen Ausbreitung neuropathologischer Veränderungen und der in vivo nachgewiesenen Volumenreduktion temporaler Strukturen hin. 


\subsubsection{Klinische Relevanz der kernspintomographischen Befunde}

Neben der Frage nach dem diskriminativen Wert des Hippocampusvolumens im Rahmen von Mittelwertsvergleichen zwischen dementen und nicht dementen Probanden interessiert auch der Zusammenhang zwischen Volumenreduktion und kognitiver Leistungsfähigkeit. Zum Beispiel wiesen Petersen und Kollegen nach, daß neuropsychologisch erhobene Lern- und Gedächtnisleistungen mit dem Hippocampusvolumen in der Kontrollgruppe wie auch in der (Alzheimer-) Patientengruppe korrelierten, in letzterer sogar höher (Petersen et al., 2000). In einer anderen Studie von Barber et al. hingegen korrelierte nicht das deutlich im Vergleich zur Kontrollgruppe reduzierte Hippocampusvolumen mit Gedächtnisleistungen, sondern vielmehr Volumina lateraler Temporallappenstrukturen (Barber et al., 2001). Dieses Ergebnis erhält Bestätigung durch den Befund einer PET-Studie von Desgranges und Kollegen: Sie konnten zeigen, daß sich Zusammenhänge zwischen Hippocampus und Gedächtnis im Verlauf der Alzheimer-Erkrankung verschieben. So korrelierte bei Patienten mit fortgeschrittener Erkrankung die Bearbeitung einer story-recall-Aufgabe (ähnlich wie Logical memory der WMS-R) mit linken temporalen Assoziationscortices, nicht jedoch mit limbischen Strukturen. Sie interpretieren diesen Shift als Zeichen eines (unzureichenden) Kompensationsmechanismus, in dem zur Bearbeitung einer Aufgabe, die normalerweise limbische Regionen aktiviert hätte, neocorticale Regionen rekrutiert werden, da die limbischen Regionen bereits in zu großem Ausmaß pathologisch verändert sind (Desgranges et al., 2002).

Die heterogenen Befunde zeigen, daß Auswirkungen von Volumenreduktionen im Rahmen eines progredienten Abbauprozesses nicht verglichen werden können mit denen umschriebener Makroinfarkte in corticalen Regionen oder subcorticalen Kerngebieten sowie gezielt gesetzten Läsionen z.B. im Rahmen der neurochirurgischen Behandlung einer medikamentenresistenten Epilepsie. Neuropsychologische Läsionsstudien mit letztgenannten Patienten oder tierexperimentelle Läsionsstudien haben zum Ziel, das hirnorganische Korrelat spezifischer kognitiver Funktionen zu lokalisieren. Korrelationsanalysen bei Patienten mit Verdacht auf eine degenerative hirnorganische Beeinträchtigung hingegen stellen eher den Versuch dar, die klinische Relevanz der Volumenreduktionen nachzuweisen. D.h. Zusammenhänge zwischen Volumenreduktion und kognitiven Defiziten weisen auf eine gemeinsame pathologische Varianz hin, nicht aber auf eine Lokalisation der kognitiven Funktion in der volumenreduzierten Hirnstruktur. Ein weiteres Interesse der Forschung zur AlzheimerDemenz besteht darin, den prädiktiven Wert verminderten Hippocampusvolumens zu bestimmen. Leichte Beeinträchtigungen der Gedächtnisfunktion ohne weitergehende kogniti- 
ve Defizite (=MCI) sind häufig bei älteren Menschen anzutreffen, aber nur etwa die Hälfte der Betroffenen entwickelt eine Demenz (Flicker, Ferris \& Reisberg, 1991; Kluger, Ferris, Golomb, Mittelman \& Reisberg, 1999). Einen Prädiktor zu finden, der diejenigen MCIPatienten identifiziert, die mit höherer Wahrscheinlichkeit eine Alzheimer-Demenz entwickeln werden, ist von ungeheuer großem diagnostischen Wert. Es gibt zur Zeit keine Möglichkeit der ursächlichen Behandlung der Alzheimer-Demenz, jedoch haben medikamentöse Therapien eine Verbesserung kognitiver Leistungen oder eine Verlangsamung der Progredienz bewirken können (Felician \& Sandson, 1999). Langzeitstudien mit MCI-Patienten konnten den prädiktiven Wert der Hippocampus-Atrophie eindeutig nachweisen: Patienten mit MCI und geringerem Hippocampusvolumen zum ersten Testzeitpunkt entwickelten in der Folgezeit häufiger eine klinisch manifeste Alzheimer-Demenz als Patienten, die lediglich Gedächtnisbeeinträchtigungen aufwiesen (Jack et al., 1999; Jack et al., 2000; Visser, Verhey, Hofman, Scheltens \& Jolles, 2002; Yamaguchi et al., 2002).

\subsubsection{Zusammenhang ZWischen Alzheimer-Demenz und Koronarer Herz- KRANKHEIT}

Die Diagnose einer Alzheimer-Demenz beinhaltet den Ausschluß alternativer Erklärungen für die kognitiven Defizite (Saß, Wittchen \& Zaudig, 1996). Das Vorliegen cerebrovaskulärer Risikofaktoren und noch mehr das Vorliegen cerebrovaskulärer Befunde im Kernspintomogramm schließt also die Diagnose einer Alzheimer-Demenz aus. Das führte in der Vergangenheit dazu, daß Hinweise auf gemeinsame Pathologien von Alzheimer- und vaskulärer Demenz übersehen wurden. 1990 veröffentlichten Sparks und Mitarbeiter Ergebnisse einer neuropathologischen Studie, die bisher unentdeckte Zusammenhänge ins Licht rückte: In Gehirnen nicht-dementer Patienten mit KHK konnten neuropathologische Veränderungen, d.h. senile Plaques und neurofibrilläre Degenerationen, im medialen Temporallappen nachgewiesen werden, die denen in Gehirnen von Alzheimer-Patienten glichen (Sparks et al., 1990). In der Folgezeit konnte das Ergebnis in weiteren Studien repliziert werden (Soneira \& Scott, 1996; Sparks, Liu, Scheff, Coyne \& Hunsaker, 1993; Sparks et al., 1995). Inzwischen weisen andere Studien auf Zusammenhänge zwischen Alzheimer-Demenz und Koronarer Herzkrankheit bzw. vaskulären Risikofaktoren hin: Prospektive Studien zeigen Korrelationen zwischen Bluthochdruck und Entwicklung einer Demenz im späteren Leben (Launer, Masaki, Petrovitch, Foley \& Harvik, 1995; Petrovitch et al., 2000; Skoog et al., 1996), andere weisen arteriosklerotische Veränderungen bei Patienten mit Alzheimer-Demenz nach (Hofman et al., 1997; Sparks et al., 1995). Breteler und Mitarbeiter identifizierten das Vorliegen einer KHK 
als Risikofaktor für die Entwicklung einer Alzheimer-Demenz (Breteler, Claus, Grobbee \& Hofman, 1994).

Die Interpretation dieser Befunde ist noch nicht abgeschlossen (Skoog, 2000): Addieren sich cerebrovaskuläre und Alzheimer-typische Veränderungen des Gehirns und führen in ihrer Summe oder Interaktion zu funktionellen Beeinträchtigungen? Weiterhin stellt sich die Frage, ob zwei unabhängige Pathomechanismen existieren oder beide Prozesse einer gemeinsamen Ätiologie unterliegen. Petrovitch und Kollegen argumentieren für eine direkte Verursachung der Alzheimer-typischen Neuropathologie durch vaskuläre Risikofaktoren : Craniale Arteriosklerose bei hypertensiven Patienten könnte zu einer Kumulierung chronisch-hypoxischer Läsionen führen, die Auslöser für die neuropathologischen Veränderungen bei Alzheimer sind (Petrovitch et al., 2000). Sparks und Mitarbeiter spekulieren, daß Bluthochdruck/ Koronare Herzkrankheit "neuropathologic forerunner" der Alzheimer-Demenz sein könnten (Sparks et al., 1995): Die neuropathologischen Befunde sind bei beiden Erkrankungen identisch und könnten einer gemeinsamen Pathologie unterliegen. Als gemeinsame pathophysiologische Basis diskutieren Sparks et al. sogenannte freie Radikale und oxidativen Streß, physiologische Prozesse, die bei Bluthochdruck/Koronarer Herzkrankheit, aber auch bei Alzheimer eine Rolle spielen (Liu, Cuddy \& Peirce, 1992; Volicer \& Crino, 1990).

\subsection{Fragestellung UND AbLEITUNG DER HYPOTHESEN}

Epidemiologische Studien haben wiederholt gezeigt, daß kardiovaskuläre Risikofaktoren und Erkrankungen mit einer schlechteren kognitiven Leistungsfähigkeit assoziiert sind und das Risiko erhöhen, eine Demenz zu entwickeln. Bisher wurde die kognitive Leistungsfähigkeit in diesen Studien fast aussschließlich mit globalen Screening-Instrumenten erfaßt. Es deuten sich allerdings Einbußen vor allem im Gedächtnis- und Aufmerksamkeitsbereich sowie beim schlußfolgernden Denken an. Durch den Einsatz verschiedener anerkannter neuropsychologischer Testverfahren soll differenzierter als bisher überprüft werden, in welchen Funktionsbereichen sich schlechtere kognitive Leistungen von Patiententen mit KHK zeigen. Zusätzlich soll mittels einer experimentellen Aufgabe zum implizit-motorischen Lernen überprüft werden, ob Patienten mit Koronarer Herzkrankheit nicht nur beim expliziten Speichern von Gedächtnisinhalten, sondern auch beim impliziten Lernen einer motorischen Sequenz beeinträchtigt sind. 


\section{Neuropsychologische Testleistungen}

H1a: Bezüglich des intellektuellen Funktionsniveaus zeigen Patienten mit Koronarer Herzkrankheit schlechtere Leistungen als gesunde Kontrollprobanden in Tests, die schlußfolgerndes Denken überprüfen.

H1b: Bei expliziten Lern- und Gedächtnisaufgaben zeigen Patienten mit Koronarer Herzkrankheit schlechtere Leistungen als gesunde Kontrollprobanden.

H1c: Bei der Überprüfung impliziter Lernleistungen schneiden Patienten im Vergleich mit gesunden Kontrollprobanden schlechter ab.

H1d: Im Bereich der Informationsverarbeitung weisen Patienten mit Koronarer Herzkrankheit schlechtere Leistungen als gesunde Kontrollprobanden auf.

Kernspintomographisch identifizierbare White Matter Hyperintensitäten sind häufig Zufallsbefunde bei Probanden in höherem Lebensalter. Neben dem Lebensalter konnte eine Vielzahl empirischer Untersuchungen eine erhöhte Prävalenz von Patienten mit vaskulären Risikofaktoren bzw. einer KHK für diese kernspintomographisch identifizierbaren Läsionen im Gehirn zeigen. Auch die Prävalenzrate für subklinische lakunäre Infarzierungen ist bei Probanden mit vaskulären Risikofaktoren bzw. einer KHK im Vergleich zu gesunden Probanden erhöht.

\section{White Matter Hyperintensitäten und lakunäre Infarzierung}

H2a: Patienten mit einer Koronaren Herzkrankheit weisen häufiger höhergradige White Matter Hyperintensitäten auf als gesunde Kontrollprobanden.

H2b: Patienten mit einer Koronaren Herzkrankheit haben häufiger lakunäre Infarzierungen als Kontrollprobanden.

H2c: Sowohl bei Patienten mit Koronarer Herzkrankheit als auch bei gesunden Kontrollprobanden korreliert das Lebensalter positiv mit dem Schweregrad der White Matter Hyperintensitäten.

H2d: Sowohl in der Patientengruppe als auch in der Kontrollgruppe korreliert der Schweregrad der White Matter Hyperintensitäten negativ mit neuropsychologischen Testleistungen. 
Neuropathologische Studien konnten in Gehirnen von Patienten mit vaskulären Risikofaktoren bzw. einer KHK Alzheimer-typische neuropathologische Veränderungen im medialen Temporallappen feststellen. In vivo korrespondieren diese Veränderungen bei AlzheimerPatienten mit Volumenreduktionen medialer Temporallappenstrukturen, vor allem des Hippocampus, ohne daß es bereits zu einer globalen Atrophie des Gehirns gekommen ist. Neuropathologische Studien zeigen ebenfalls, daß sich die Alzheimer-typischen Veränderungen in einer klar definierten Weise ausbreiten. Während zunächst nur Teile des allocorticalen Temporallappens betroffen sind, sind später auch isocorticale Temporallappenstrukturen beeinträchtigt. Im Endstadium lassen sich die neuropathologischen Veränderungen im gesamten Gehirn finden. Auf dem Wege eines hypothesengenerierenden Vorgehens soll überprüft werden, ob sich die Ausbreitung der Pathologie bei nicht-dementen Patienten mit KHK bereits ankündigt. Es soll überprüft werden, ob das Hippocampusvolumen eine gemeinsame (pathologische) Varianz mit anderen Temporallappenstrukturen wie dem temporalen Pol und dem neocorticalen Temporallappen aufweist und ob sich bereits Volumenunterschiede in diesen Strukturen zwischen Patienten und Kontrollprobanden zeigen. Da angenommen wird, daß die Volumenreduktion des Hippocampus sowie die Volumenreduktion bzw. pathologische Varianz der Volumina weiterer Temporallappenstrukturen wie dem temporalen Pol und dem neocorticalen Temporallappen in einem Zusammenhang mit schlechteren kognitiven Leistungen stehen, werden in der Patientengruppe Korrelationen zwischen den Volumina temporaler Strukturen und Leistungen in neuropsychologischen Tests, in denen die Patienten im Mittelwertsvergleich schlechtere Ergebnisse erzielten, erwartet.

\section{Volumenreduktion von Temporallappenstrukturen}

H3a: Patienten mit Koronarer Herzkrankheit weisen kleinere Hippocampusvolumina als gesunde Kontrollprobanden auf.

H3b: Patienten mit Koronarer Herzkrankheit zeigen kleinere Volumina weiterer Temporallappenstrukturen wie dem temporalen Pol und dem neocorticalen Temporallappen im Vergleich mit gesunden Kontrollprobanden.

H3c: Bei Patienten mit Koronarer Herzkrankheit korreliert das Volumen des Hippocampus gleichsinnig mit dem des temporalen Pols und dem des neocorticalen Temporallappens. 
H3d: Bei Patienten mit Koronarer Herzkrankheit hängen kleinere Volumina temporaler Strukturen wie Hippocampus, temporaler Pol und neocorticaler Temporallappen mit defizitären neuropsychologischen Testleistungen zusammen. 


\section{METHODEN}

\subsection{Patienten Und KontrollPRobanden}

\subsubsection{Patienten Mit Koronarer HERZKRANKHEIT}

\subsubsection{Rekrutierung}

In Zusammenarbeit mit der Abteilung Thorax-, Herz- und Gefäßchirurgie (THG) und der Abteilung Kardiologie des Göttinger Universitätsklinikums wurden Patienten untersucht, die folgende Einschlußkriterien erfüllten:

- $\quad$ sichere Diagnose einer KHK,

- $\quad$ nicht älter als 70 Jahre,

- Deutsch als Muttersprache,

- $\quad$ keine neurologischen oder psychiatrischen Erkrankungen

- $\quad$ und bisher noch keine aortokoronare Venenbypass-Operation (ACVB) unter Einsatz der Herz-Lungen-Maschine.

Patienten der THG: In der Zeit von Juni 1999 bis Februar 2001 wurden ca. 120 stationäre Patienten der Thorax-, Herz- und Gefäßchirurgie kurze Zeit nach ihrer Aufnahme zur ACVB-Operation angesprochen. Etwa die Hälfte dieser Patienten erfüllte die Einschlußkriterien und wurde über die Studie aufgeklärt (s. Anhang A). Bei ca. einem Viertel der Patienten $(n=29)$ bestand die Bereitschaft zur Teilnahme. Die Patienten wurden dann im Verlauf der präoperativen Phase an zwei Nachmittagen in den Räumen der THG bzw. der Psychiatrischen Klinik untersucht.

Patienten der Kardiologie: In der Zeit von Februar bis August 2001 wurden Befundberichte der Abteilung für Kardiologie gesichtet. Geeignete Patienten wurden angeschrieben (s. Anhang B), nachdem ihr stationärer Aufenthalt beendet war. Die Aufnahmedauer dieser Patienten reichte nicht für eine Studienteilnahme während des stationären Aufenthalts aus, da sie bereits kurze Zeit nach der Diagnosestellung mittels Herzkathetheruntersuchung (Koronarangiographie) entlassen wurden. Von 45 angeschriebenen Patienten nahmen schließlich 15 an der Studie teil. Die übrigen sagten entweder aus verschiedenen Gründen ab (mangelndes Interesse, Angst vor der kernspintomographischen Untersuchung, zu schlechter Allgemeinzu- 
stand) oder wurden nach einer kurzen telephonischen Exploration ausgeschlossen, da sie die Einschlußkriterien nicht erfüllten. Die Untersuchung wurde an einem Nachmittag in den Räumen der Psychiatrischen Klinik durchgeführt. Den ambulanten Patienten wurden die Fahrtkosten erstattet.

Insgesamt wurden 44 Patienten rekrutiert. Sieben Patienten wurden von der Datenanalyse ausgeschlossen, fünf wegen fehlender kernspintomographischer Untersuchung. Eine Patientin wurde wegen einer im Kernspintomogramm ersichtlichen und von einem Neurochirurgen verifizierten Temporallappendysplasie ausgeschlossen, bei einem weiteren Patienten wurde die Untersuchung abgebrochen, da sich der Verdacht auf einen Alkoholabusus ergeben hatte. Somit gingen 37 Patienten mit KHK in die Studie ein.

\subsubsection{Krankheitsbezogene Variablen}

Die zum Zeitpunkt der Untersuchung bereits von allen Patienten vorliegenden koronarangiographischen Untersuchungsergebnisse zeigten, daß drei der 37 Patienten unter einer 1Gefäß-KHK litten, neun unter einer 2-Gefäß-KHK und 25 unter einer 3-Gefäß-KHK. 17 der 37 Patienten hatten mindestens einen Herzinfarkt in der Anamnese. 23 der 37 Patienten waren für eine aortokoronare Venenbypass-Operation vorgesehen.

Tabelle 1: Krankheitsbezogene Angaben zu den KHK-Patienten

\begin{tabular}{|c|c|c|}
\hline \multicolumn{3}{|c|}{ Anzahl betroffener Gefäße } \\
\hline \multicolumn{2}{|c|}{ 1-Gefäß-KHK } & $(8 \%)$ \\
\hline \multicolumn{2}{|c|}{ 2-Gefäß-KHK } & $(24 \%)$ \\
\hline \multicolumn{2}{|c|}{ 3-Gefäß-KHK } & $(68 \%)$ \\
\hline \multicolumn{2}{|c|}{ mind. 1 Herzinfarkt in der Anamnese } & $(46 \%)$ \\
\hline \multicolumn{3}{|c|}{ Therapie } \\
\hline \multicolumn{2}{|r|}{ nicht-operativ (medikamtenös; PTCA) } & $(38 \%)$ \\
\hline \multicolumn{2}{|c|}{ für ACVB vorgesehen } & $(62 \%)$ \\
\hline $\begin{array}{l}\text { KHK } \\
\text { PTCA } \\
\text { ACVB }\end{array}$ & $\begin{array}{l}\text { Koronare Herzkrankheit } \\
\text { perkutane transluminale koronare } \\
\text { Aortokoronarer Venenbypass }\end{array}$ & \\
\hline
\end{tabular}

\subsubsection{KONTROLLPROBANDEN}

Als Kontrollgruppe wurde eine Stichprobe gesunder Probanden ( $n=24)$ untersucht, die über eine Annonce in einer regionalen Zeitung sowie über Aushänge und Handzettel in Göt- 
tingen angeworben worden waren. Es wurden nur Probanden untersucht, die weder neurologische noch psychiatrische Erkrankungen in der Anamnese bzw. zum Zeitpunkt der Studienteilnahme aufwiesen. Die Diagnose einer KHK sowie anderer kardialer Erkrankungen stellte ebenfalls einen Ausschlußgrund dar. Die Probanden erhielten für ihre Teilnahme eine Aufwandsentschädigung in Höhe von ca. $75,-€$.

\subsubsection{Vergleich Der Patienten mit Den Kontrollprobanden: SOziodemo- GRAPHISCHE VARIABLEN UND AUSMAß DEPRESSIVER SYMPTOMATIK}

Es gab keine bedeutsamen Unterschiede zwischen den KHK-Patienten und den Kontrollprobanden hinsichtlich des Alters, der Schul- und Berufsausbildung in Jahren oder der erreichten Schulabschlüsse. Das Beck-Depressions-Inventar BDI (Hautzinger, Bailer, Worall \& Keller, 1995) wurde von allen Studienteilnehmern bis auf einen Patienten ausgefüllt. Das Ausmaß der depressiven Beeinträchtigung im $B D I$ war in beiden Gruppen vergleichbar gering und erreichte weder in der Patienten- noch in der Kontrollgruppe klinische Bedeutsamkeit. Mit Mittelwerten von 6,2 bzw. 4,9 Punkten lagen beide deutlich unter der Grenze für eine leichte depressive Symptomatik (=11 Punkte). Eine Beeinträchtigung kognitiver Funktionen durch eine schwerwiegende depressive Verstimmung konnte demnach in beiden Gruppen ausgeschlossen werden.

Die Geschlechterverteilung in der Kontrollgruppe wurde der in der Patientengruppe angepaßt, so daß sich hier ebenfalls kein bedeutsamer Unterschied ergab. Bei der Rekrutierung der KHK-Patienten hatte es sich als unmöglich erwiesen, Frauen und Männer in gleichen Anteilen zu erheben, ohne daß sie sich hinsichtlich bedeutsamer Variablen wie Alter und Komorbiditäten unterschieden. Aus Studien mit breiter angelegten Erhebungen ist das größere Risiko von Männern für kardiovaskuläre Erkankungen bekannt (Mittelmark et al., 1993). Zusätzlich zur ungleichen Verteilung kardiovaskulärer Erkrankungen in der Grundgesamtheit zeigt sich, daß Frauen bei Diagnosestellung einer kardialen Erkankung ein deutlich höheres Lebensalter aufweisen als Männer (Lagerquist, Safstrom, Stahle, Wallentin \& Swahn, 2001). Dies wird auf die protektive Wirkung der Östrogene auf das vaskuläre System zurückführt (Schwertz \& Penckofer, 2001). Manche Studien zeigen jedoch auch, daß Ärzte kardiale Erkankungen bei Frauen häufig unterdiagnostizieren, so daß ihre Erkrankung im Vergleich zu männlichen Patienten mit ähnlichem Beschwerdekomplex erst später erkannt wird (Jong \& Sternberg, 1998; Keller \& Lemberg, 1998). In der vorliegenden Studie wurden fünf Frauen und 39 Männer mit KHK untersucht. Da drei Frauen von der Studie ausgeschlossen wurden 
(zwei wegen eines fehlenden MRT, eine wegen einer Temporallappendysplasie), verblieben lediglich zwei Frauen in der Patientengruppe. Die zwei weiblichen Kontrollprobanden entsprachen den Patientinnen in Alter und Schulausbildung. Aufgrund der geringen Fallzahlen wird von einer geschlechtsdifferentiellen Analyse abgesehen.

Tabelle 2: Soziodemographische Variablen und Ausmaß der depressiven Symptomatik der Patienten und Kontrollprobanden

\begin{tabular}{|c|c|c|c|c|}
\hline & $\begin{array}{c}\text { KHK-Patienten } \\
\qquad(\mathrm{n}=\mathbf{3 7})\end{array}$ & $\begin{array}{c}\text { Kontrollprobanden } \\
\qquad(\mathrm{n}=\mathbf{2 4})\end{array}$ & Prüfgröße & Signifikanz \\
\hline & a & $\mathrm{a}$ & & $p$ \\
\hline Alter (in Jahren) ${ }^{1}$ & $60.5 \quad(5.2)$ & $60.9 \quad(5.9)$ & $t[59]=-0.28$ & n.s. \\
\hline Bildung (in Jahren) ${ }^{1}$ & $13.1 \quad(3.3)$ & $12.9(3.7)$ & $t[59]=0.27$ & n.s. \\
\hline Schulabschluß (VS : RS : AB), $\mathrm{N}^{2}$ & $21: 7: 9$ & $16: 4: 4$ & & n.s. \\
\hline Geschlecht $(F: M), N^{2}$ & $2: 35$ & $2: 22$ & & n.s. \\
\hline BDI $^{1}$ & $6.2(4.6)$ & $4.9 \quad(3.5)$ & $t[58]=1.21$ & n.s. \\
\hline
\end{tabular}

Legende: ${ }^{a}$ Mittelwert und Standardabweichung, wenn nicht anders angegeben; Bildung: Dauer der Schul- und Berufsausbildung; N: Häufigkeit; VS: Volksschule; RS: Realschule; AB: Abitur; F: Frauen; M: Männer; ; BDI: Beck-Depressions-Inventar; ${ }^{1} t$-Test (zweiseitig); ${ }^{2}$ exakter Test nach Fisher und Yates (zweiseitig)

\subsubsection{Vergleich Der Patienten mit Den Kontrollprobanden: Verteilung VASKULÄRER RISIKOFAKTOREN}

Aus epidemiologischen Studien sind als Risikofaktoren für pathologische Veränderungen des vaskulären Systems Hypertonie, Hypercholesterinämie und Diabetes mellitus bekannt. Weitere für vaskulär bedingte Erkrankungen disponierende Faktoren sind Adipositas und Nikotinabsus (Schanzenbächer \& Kochsiek, 1993). In der vorliegenden Studie wurde das Vorhandensein der Risikofaktoren Hypertonie, Hypercholesterinämie bzw. Diabetes mellitus anhand der Medikation entschieden, eine in der Forschung übliche Praxis (Kalmijn, Fesken, Launer \& Kromhout, 1996; Knopman et al., 2001; Tzourio, Dufouil, Ducimetière \& Alpérovitch, 1999). Bei Patienten wurde auf in Krankenakten verfügbare Informationen bezüglich der Medikation zurückgegriffen, die Kontrollprobanden wurden gebeten, eine Aufstellung ihrer aktuellen Medikation zur Untersuchung mitzubringen. 35 der 37 KHK-Patienten und fünf der 24 Kontrollprobanden nahmen zum Zeitpunkt der Untersuchung ein bzw. mehrere Medikamente der Klasse Antihypertonika ein. Medikamente gegen hohe Blutfette hingegen 
waren 25 der 37 KHK-Patienten verschrieben, aber niemandem in der Kontrollgruppe. Unter Diabetes mellitus litten vier der Patienten und einer der Kontrollprobanden.

Über derzeitiges Körpergewicht in kg und Körpergröße in m wurde für jeden Untersuchungsteilnehmer der sog. Body Mass Index (BMI) über die Formel kg/m² berechnet. Als klinisch bedeutsames Übergewicht (=Adipositas) gilt ein BMI > 30 (Bray, 1978). In der vorliegenden Untersuchung galten demnach 12 der 37 Patienten und vier der 24 Kontrollprobanden als adipös. Bezüglich des Nikotinabusus wurden alle Probanden befragt, ob sie in den letzten 20 Jahren über einen Zeitraum von mindestens 15 Jahren regelmäßig und wenn ja, wieviele Zigaretten täglich, geraucht haben (Schanzenbächer \& Kochsiek, 1993). Sechs der KHKPatienten und sieben der Kontrollprobanden hatten über mindestens 15 Jahre zwischen 1980 und 2000 täglich mehr als 20 Zigaretten geraucht.

Ein Vergleich der Verteilung der Risikofaktoren im $\chi^{2}$-Quadrat-Test ergab lediglich für die Risikofaktoren Hypertonie und Hypercholesterinämie eine signifikant höhere Häufigkeit in der Patientengruppe $\left(\chi^{2}=31.9 ; p<0.01\right.$ bzw. $\left.\chi^{2}=21.4 ; p<0.01\right)$. Bezüglich des Vorhandenseins von Diabetes mellitus, Adipositas und Nikotinabusus ergaben sich keine statistisch signifikanten Häufigkeitsunterschiede zwischen Patienten- und Kontrollgruppe.

Tabelle 3: Verteilung vaskulärer Risikofaktoren bei Patienten und Kontrollprobanden

\begin{tabular}{|c|c|c|c|c|}
\hline & $\begin{array}{c}\text { KHK-Patienten } \\
\qquad(n=37)\end{array}$ & $\begin{array}{l}\text { Kontrollprobanden } \\
\qquad(n=24)\end{array}$ & Prüfgröße & Signifikanz \\
\hline & ja : nein & ja : nein & & $p$ \\
\hline Hypertonie $^{\text {a, } 1}$ & $35: 2$ & $5: 19$ & $\chi^{2}=31.9$ & $<0.01$ \\
\hline Hypercholesterinämie $^{\mathrm{a}, 1}$ & $23: 14$ & $0: 24$ & $\chi^{2}=21.4$ & $<0.01$ \\
\hline Diabetes mellitus $^{\text {a, } 2}$ & $4: 33$ & $1: 23$ & & n.s. \\
\hline $\operatorname{Adipositas}^{\mathrm{b}, 1}$ & $12: 25$ & $4: 20$ & $\chi^{2}=1.1$ & n.s. \\
\hline Nikotin $^{\text {c, } 1}$ & $6: 31$ & $7: 17$ & $\chi^{2}=0.8$ & n.s. \\
\hline
\end{tabular}

Legende: ${ }^{1}$ korrigiertes $\chi^{2}$-Quadrat nach Yates (zweiseitig); ${ }^{2}$ exakter Test nach Fisher und Yates (zweiseitig); ${ }^{a}$ nach Medikation; ${ }^{\mathrm{b}}$ Body-Mass-Index (Körpergwicht in $\mathrm{kg} /$ Körpergröße in $\mathrm{m}^{2}$ ) >30; ${ }^{\mathrm{c}}$ ab 1980 mind. 15 Jahre mind. 20 Zigaretten/Tag 


\subsection{UNTERSUCHUNGSVERFAHREN}

\subsubsection{NEUROPSYCHOLOGISCHE UNTERSUCHUNGSVERFAHREN}

Ziel der neuropsychologischen Untersuchung war es, durch den Einsatz international anerkannter Testverfahren ein breites Spektrum kognitiver Funktionen zu erfassen. Es sollten verschiedene intellektuelle Fähigkeiten (s. 2.2.1.1), explizite und implizite Gedächtnisleistungen (s. 2.2.1.2) sowie differentielle Leistungen in der Informationsverarbeitung (s. 2.2.1.3) der KHK-Patienten erhoben und mit denen der Kontrollprobanden verglichen werden.

\subsubsection{Erfassung intellektueller Fähigkeiten}

Allgemeines Wissen (HAWIE-R): Es werden aufeinanderfolgend 24 Fragen mit ansteigendem Schwierigkeitsgrad gestellt. Die Fragen umfassen Bereiche des überlernten Schulund Weltwissens. Nach fünf aufeinanderfolgenden nicht bzw. falsch beantworteten Fragen wird der Test abgebrochen. In die statistische Analyse geht der erreichte Punktwert (=Anzahl richtig beantworteter Fragen) ein. Der maximal erreichbare Wert beträgt 24 Punkte.

Gemeinsamkeitenfinden (HAWIE-R): Es werden in insgesamt 16 Aufgaben jeweils zwei Dinge oder Begriffe genannt, die eine Gemeinsamkeit aufweisen oder unter einem Oberbegriff zusammengefaßt werden können. Die Lösung kann konkret (z.B. Mantel-Anzug=sind aus Stoff) oder logisch-abstrakt (z.B. Mantel-Anzug=Bekleidung) sein. Für konkrete Antworten wird ein Punkt, für logisch-abstrakte werden zwei Punkte vergeben. Nach vier aufeinanderfolgenden nicht bzw. falsch beantworteten Fragen wird abgebrochen. In die statistische Analyse geht der erreichte Punktwert (=Summe der vergebenen Punktzahlen) ein. Der maximal erreichbare Wert beträgt 32 Punkte.

Bilderergänzen (HAWIE-R): Es werden insgesamt 17 Bilder vorgelegt, auf denen jeweils ein bedeutsames Detail fehlt. Fehlende Details können konkret (z.B. das Fehlen eines eines Beines bei einem Frosch) oder logisch-abstrakt (z.B. ein fehlerhaftes Spiegelbild) sein. Nach drei aufeinanderfolgenden nicht bzw. falsch beantworteten Aufgaben wird abgebrochen. In die statistische Analyse geht der erreichte Punktwert (=Anzahl richtig beantworteter Aufgaben) ein. Der maximal erreichbare Wert beträgt 17 Punkte.

Mosaiktest (HAWIE-R): Mit vier (Aufgabe 1-5) bzw. neun (Aufgabe 6-9) mehrfarbigen Würfeln sollen auf Kärtchen abgebildetete Muster so schnell wie möglich nachgebildet werden. Es wird die Zeit gestoppt, die für das korrekte Nachbilden benötigt wird. Es werden für 
unterschiedliche Zeitgrenzen unterschiedlich hohe Punktzahlen vergeben. Nach drei Fehlversuchen in Folge wird abgebrochen. In die statistische Analyse geht der erreichte Punktwert (=Summe der pro Aufgabe erzielten Punktzahlen) ein. Der maximal erreichbare Wert beträgt 51 Punkte.

\subsubsection{Erfassung expliziter und impliziter Lern- und Gedächtnisleistungen}

Serielle Reaktionszeitsaufgabe: Diese experimentelle Aufgabe wurde eingesetzt, um das implizite Erlernen einer motorischen Sequenz zu erfassen. Die in der vorliegenden Studie eingesetzte Version stellt eine Modifikation der Originalaufgabe von Nissen und Bullemer (1987) dar. In der Aufgabe werden an vier feststehenden Positionen des Bildschirms Stimuli (Sternchen) präsentiert. Der Proband soll so schnell wie möglich auf die korrespondierende Reaktionstaste drücken. Der Proband hat keine Kenntnis davon, daß es sich bei der Abfolge der Positionen um eine wiederkehrende Sequenz handelt. Es wurden sechs Blöcke mit jeweils 120 Versuchsdurchgängen dargeboten. In Block 1 und Block 5 wurden die Positionen des Sterns zufällig gewählt, in Block 2 bis 4 und in Block 6 hingegen folgten die Positionen der Sequenz. Es konnte konsistent und replizierbar gezeigt werden, daß die Reaktionszeiten über die Blöcke kleiner werden, da der Proband die Sequenz implizit lernt und somit schneller reagieren kann, ohne daß er ein explizites Wissen über die Sequenz erlangt (Seger, 1994). Das Ausmaß des impliziten Lernens wird durch Differenzbildung zwischen den mittleren Reaktionszeiten in Block 4 (Sequenz) und Block 5 (Zufall) ermittelt. Der Parameter Lerngeschwindigkeit (mittlere Reaktionszeit in Block 1 minus mittlere Reaktionszeit in Block 2) gibt an, wie schnell der Proband von der Sequenz-Abfolge profitiert, d.h. in welchem Ausmaß er bereits im ersten Sequenz-Block (=Block 2$)$ von der Sequenz profitieren kann (vgl. Exner, Weniger \& Irle, 2001).

Logical Memory I und II (WMS-R): Im direkten Anschluß an das Vorlesen einer Geschichte soll diese nacherzählt werden (sofortiger Abruf). Nach ca. 30 Minuten soll die geschichte erneut reproduziert werden (verzögerter Abruf). Zu dem Untertest gehören zwei Geschichten, die in insgesamt 50 semantische Einheiten aufgeteilt sind. Pro nacherzählter semantischer Einheit wird ein Punkt vergeben, dazu ist die wörtliche oder inhaltlich analoge Nennung nötig, z.B. "waren gerührt" als wörtliche, "hatten Mitleid" als inhaltlich analoge Nennung. In die statistische Analyse geht die Summe der richtigen Nennungen über beide Geschichten ein. Der maximal ereichbare Punktwert beträgt für den sofortigen und verzögerten Abruf jeweils 50 Punkte. 
Verbal Paired Associates I und II (WMS-R): Im sofortigen Abruf werden acht Wortpaare vorgelesen, von denen das jeweils zweite bei der anschließenden Vorgabe des jeweils ersten Wortes erinnert werden soll. Vier der Wortpaare sind semantisch assoziiert (z.B. RoseBlume), die anderen vier sind es nicht (z.B. Salat-Stift). Pro richtiger Antwort wird ein Punkt vergeben. Es werden drei Durchgänge durchgeführt, d.h. der maximal erreichbare Wert im sofortigen Abruf beträgt 24 Punkte. Die Aufgabe wird nach drei Durchgängen abgebrochen, wenn im dritten Durchgang alle acht Antworten richtig sind. Ansonsten werden bis zu maximal drei weiteren Durchgänge durchgeführt. Es wird abgebrochen, sobald ein Durchgang vollständig richtig beantwortet wurde. Im verzögerten Abruf wird ohne erneutes Vorlesen der Wortpaare bei Nennung des jeweils ersten nach dem zweiten Wort gefragt. Der maximal erreichbare Wert beträgt hier acht Punkte.

Visual Paired Associates I und II (WMS-R): Im sofortigen Abruf werden acht Paare bestehend aus einer Farbe und einer Strichfigur vorgelegt. In der Abfrage wird jeweils die Strichfigur gezeigt und die dazugehörige Farbe soll in dem vorgelegtenTestheft gezeigt werden. Pro richtiger Antwort wird ein Punkt vergeben. Es werden drei Durchgänge durchgeführt, d.h. der maximal erreichbare Wert im sofortigen Abruf beträgt 18 Punkte. Die Aufgabe wird nach drei Durchgängen abgebrochen, wenn im dritten Durchgang alle sechs Antworten richtig sind. Ansonsten werden bis zu maximal drei weitere Durchgänge durchgeführt. Es wird abgebrochen, sobald ein Durchgang vollständig richtig beantwortet wurde. Im verzögerten Abruf wird ohne erneutes Vorlegen der Farbe-Strichfigur-Paare bei Vorlegen der Strichfigur nach der dazugehörigen Farbe gefragt. Der maximal ereichbare Wert beträgt hier sechs Punkte.

Visual Reproduction I und II (WMS-R): Im sofortigen Abruf wird für 10 Sekunden eine Karte mit geometrischen Figuren vorgelegt, die im Anschluß an die Präsentation sofort gezeichnet werden sollen. Insgesamt werden vier Stimuluskarten vorgelegt. Im verzögerten $A b$ ruf sollen alle vier Stimuluskarten ohne erneute Darbietung frei reproduziert werden. Es werden für jede Reproduktion Punkte nach bestimmten Auswertungskriterien vergeben. In die statistische Analyse geht die Summe der vergebenen Punktwerte über alle vier Stimuli ein. In beiden Abrufbedingungen beträgt der maximal erreichbare Wert 41 Punkte.

Figural Memory (WMS-R): Das Stimulusmaterial besteht aus rechteckig angeordneten Mustern in verschiedenen Grauschattierungen. Im ersten Durchgang soll aus drei Mustern das zuvor präsentierte Muster wiedererkannt werden. In Durchgang 2 bis 4 werden drei Muster gezeigt, die anschließend aus neun vorgelegten wiedererkannt werden sollen. Pro richtiger 
Antwort wird ein Punkt vergeben. Die Summe der vergebenen Punkte über alle vier Durchgänge geht als Punktwert in die statistische Analyse ein. Der maximal erreichbare Wert beträgt 10 Punkte.

\subsubsection{Erfassung differentieller Leistungen in der Informationsverarbeitung}

Arbeitsgedächtnis (TAP): Auf dem Bildschirm werden nacheinander einstellige Zahlen präsentiert. Es wird der Vergleich der jeweils gegebenen Zahl mit der vorletzten Zahl verlangt. Bei Übereinstimmung der aktuell dargebotenen mit der vorletzten Zahl soll so schnell wie möglich durch Tastendruck reagiert werden. Es werden 100 Reize präsentiert, von denen 15 kritisch sind. Das Programm gibt die mittlere Reaktionszeit der Antworten und Anzahl der Fehlreaktionen aus.

Geteilte Aufmerksamkeit (TAP): In der optischen Aufgabe werden jeweils acht Kreuze, die an 4x4 feststehenden Positionen auf dem Bildschirm erscheinen können, dargeboten. Bilden vier der acht Kreuze ein Quadrat, soll mittels Tastendruck reagiert werden. In der simultan durchgeführten akustischen Aufgabe werden hintereinander Töne in zwei verschiedenen Höhen dargeboten. Mittels Tastendruck soll auf die Unregelmäßigkeit der Tonfolge reagiert werden, d.h. wenn hintereinander zwei Töne derselben Höhe präsentiert werden. Es werden insgesamt 100 optische und 200 akustische Durchgänge dargeboten. Das Programm gibt die mittlere Reaktionszeit der Antworten und Anzahl der Fehlreaktionen sowie der Aulassungen aus.

Reaktionswechsel (TAP): Es werden simultan rechts und links vom Fixationspunkt in der Mitte des Bildschirms eine Zahl und ein Buchstabe präsentiert. Es soll auf diejenige der rechts und links angeordneten Reaktionstasten gedrückt werden, auf deren Seite sich der Zielreiz (Buchstabe oder Zahl) befindet. Der Zielreiz alterniert in jedem Durchgang (Buchstabe, Zahl, Buchstabe, Zahl); in manchen Durchgängen gleichsinnig mit der Darbietungsseite (rechts, links, rechts, links), in manchen Durchgängen nicht. Das Programm gibt die mittlere Reaktionszeit der Antworten und Anzahl der Fehlreaktionen aus.

Trail Making Test Form A und B: Bei Form A sollen auf einem DIN A4 großen Blatt präsentierte und mit einem Kreis markierte Zahlen in aufsteigender Reihenfolge (1-25) verbunden werden. Die gestoppte Durchführungszeit geht als Rohwert in die statistische Analyse ein. Bei Form B sollen auf einem DIN A4 großen Blatt präsentierte und mit einem Kreis markierte Zahlen und Buchstaben in aufsteigender Reihenfolge (1, A, 2, B usw. bis L, 13) alter- 
nierend verbunden werden. Die gestoppte Durchführungszeit geht als Rohwert in die statistische Analyse ein.

Digit Span Forward u. Backward (WMS-R): Es werden Zahlenfolgen in wachsender Länge vorgelesen, die jeweils im unmittelbaren Anschluß in der richtigen (Forward) bzw. in der umgekehrten Reihenfolge (Backward) wiederholt werden sollen. Die Präsentation beginnt mit dreistelligen (Forward) bzw. zweistelligen (Backward) Zahlenreihen. Die Zahlenreihen weisen maximal eine Länge von acht (Forward) bzw. sieben (Backward) Ziffern auf. Pro richtiger Reproduktion wird ein Punkt vergeben und über die insgesamt jeweils 12 Durchgänge aufsummiert. Diese Summen gehen als Punktwerte in die statistische Analyse ein. Der maximale Wert pro Untertest beträgt 12 Punkte.

Visual Memory Span Forward u. Backward (WMS-R): Es werden visuell-räumliche Anordnungen in wachsender Länge präsentiert, die jeweils im unmittelbaren Anschluß in der richtigen (Forward) bzw. in der umgekehrten Reihenfolge (Backward) wiederholt werden sollen. Dazu werden auf einem Brett unregelmäßig angeordnete Klötzchen nacheinander berührt. Die Präsentation beginnt mit drei (Forward) bzw. zwei (Backward) Klötzchen. Die Anordnungen weisen maximal eine Länge von acht (Forward) bzw. sieben (Backward) Klötzchen in Folge auf. Pro richtiger Reproduktion wird ein Punkt vergeben und über die insgesamt jeweils 12 Durchgänge aufsummiert. Diese Summen gehen als Punktwerte in die statistische Analyse ein. Der maximale Wert pro Untertest beträgt 12 Punkte.

Mental Control (WMS-R): Dieser Untertest besteht aus drei Aufgaben, die jeweils fehlerfrei und innerhalb eines bestimmten Zeitlimits bearbeitet werden sollen. In der ersten Aufgabe soll von 20 bis 1 rückwärts gezählt werden, in der zweiten soll das Alphabet aufgesagt werden, und in der dritten soll in 3er-Schritten von 1 beginnend aufwärts bis 40 gezählt werden. In jeder Aufgabe können bei richtiger Antwort innerhalb des Zeitrahmens maximal zwei Punkte erreicht werden. Die Summe der Punktwerte über die drei Aufgaben geht als Punktwert in die statistische Analyse ein. Der maximal erreichbare Summenwert beträgt sechs Punkte.

Einen Überblick über alle in der Untersuchung verwendeten Testverfahren liefert die nachfolgende Tabelle 4. 
Tabelle 4: Übersicht über die neuropsychologischen Untersuchungsverfahren (Erläuterungen zur Einteilung der Testverfahren s. Text)

\begin{tabular}{|c|c|c|c|c|}
\hline $\begin{array}{l}\text { Funktions- } \\
\text { bereich }\end{array}$ & Testverfahren & Untertest & überprüfte Leistung & $\begin{array}{l}\text { Para- } \\
\text { meter }\end{array}$ \\
\hline Intelligenz & $\begin{array}{l}\text { Hamburg- } \\
\text { Wechsler- } \\
\text { Intelligenztests } \\
\text { für Erwachsene } \\
\text { (Tewes, 1991) }\end{array}$ & $\begin{array}{l}\text { Allgemeines Wissen } \\
\text { Gemeinsamkeitenfinden } \\
\text { Bilderergänzen } \\
\text { Mosaiktest }\end{array}$ & $\begin{array}{l}\text { Allgemeinbildung } \\
\text { verbale Konzeptbildung } \\
\text { schlußfolgerndes Denken in prakti- } \\
\text { schen und konzeptionellen Bezügen } \\
\text { visuokonstruktorische Fähigkeiten }\end{array}$ & $\begin{array}{l}\text { Punkt- } \\
\text { werte }\end{array}$ \\
\hline \multirow[t]{2}{*}{$\begin{array}{l}\text { Lernen und } \\
\text { Gedächtnis }\end{array}$} & $\begin{array}{l}\text { Wechsler- } \\
\text { Memory-Scale- } \\
\text { Revised } \\
\text { (Wechsler, } \\
\text { 1987) }\end{array}$ & $\begin{array}{l}\text { Logical Memory I und II } \\
\text { Figural Memory } \\
\text { Visual Reproduction I und II } \\
\text { Verbal Paired Associates I } \\
\text { und II } \\
\text { Visual Paired Associates I } \\
\text { und II }\end{array}$ & $\begin{array}{l}\text { Einspeicherung und Abruf verbalen } \\
\text { Materials } \\
\text { Einspeicherung visuellen Materials } \\
\text { Einspeicherung und Abruf visuellen } \\
\text { Materials } \\
\text { verbales Paar-Assoziationslernen } \\
\text { (Einspeicherung und Abruf) } \\
\text { visuelles Paar-Assoziationslernen } \\
\text { (Einspeicherung und Abruf) }\end{array}$ & $\begin{array}{l}\text { Punkt- } \\
\text { werte }\end{array}$ \\
\hline & $\begin{array}{l}\text { Serielle Reak- } \\
\text { tionszeit- } \\
\text { aufgabe }\end{array}$ & $\begin{array}{l}\text { Implizites Lernen und Lern- } \\
\text { geschwindigkeit }\end{array}$ & implizites motorisches Sequenzlernen & Zeit \\
\hline \multirow[t]{3}{*}{$\begin{array}{l}\text { Differentiel- } \\
\text { le Aspekte } \\
\text { der Infor- } \\
\text { mations- } \\
\text { verarbei- } \\
\text { tung }\end{array}$} & $\begin{array}{l}\text { Wechsler- } \\
\text { Memory-Scale- } \\
\text { Revised } \\
\text { (Wechsler, } \\
\text { 1987) }\end{array}$ & $\begin{array}{l}\text { Mental Control } \\
\text { Digit Span Forward und } \\
\text { Backward } \\
\text { Visual Memory Span For- } \\
\text { ward und Backward }\end{array}$ & $\begin{array}{l}\text { Mentale Kontrolle } \\
\text { verbale Gedächtnisspanne } \\
\text { visuelle Gedächtnisspanne }\end{array}$ & $\begin{array}{l}\text { Roh- } \\
\text { werte }\end{array}$ \\
\hline & $\begin{array}{l}\text { Trail-Making- } \\
\text { Test } \quad \text { (Reitan, } \\
1992)\end{array}$ & $\begin{array}{l}\text { Teil A } \\
\text { Teil B }\end{array}$ & $\begin{array}{l}\text { visuo-motorische Suchgeschwindig- } \\
\text { keit } \\
\\
\text { viso-motorische Suchgeschwindig- } \\
\text { keit und Aufmerksamkeitswechsel }\end{array}$ & Zeit \\
\hline & $\begin{array}{l}\text { Testbatterie zur } \\
\text { Aufmerksam- } \\
\text { keitsprüfung } \\
\text { (Zimmermann } \\
\text { \& Fimm, 1993) }\end{array}$ & $\begin{array}{l}\text { Arbeitsgedächtnis } \\
\text { Geteilte Aufmerksamkeit } \\
\text { Reaktionswechsel }\end{array}$ & $\begin{array}{l}\text { kontrollierte und aufmerksamkeitsge- } \\
\text { steuerte Informationsverarbeitung } \\
\text { bimodale Aufmerksamkeit } \\
\text { Wechsel des Aufmerksamkeitsfokus }\end{array}$ & $\begin{array}{l}\text { Zeit; } \\
\text { Fehler/ } \\
\text { Auslas- } \\
\text { sungen }\end{array}$ \\
\hline
\end{tabular}




\subsubsection{KERNSPINTOMOGRAPHISCHE UNTERSUCHUNG}

Im Anschluß an die neuropsychologische Untersuchung wurde bei allen Patienten und Kontrollprobanden ein craniales Kernspintomogramm in der Abteilung Röntgendiagnostik III der Universitätsklinik Göttingen erhoben (1.5 T Philips Gyroscan). Die Aufnahmeeinstellungen der $\mathrm{T}_{1}$-gewichteten dreidimensionalen Sequenz waren standardisiert $($ Echo-Zeit $=6.0$; Wiederholungszeit $=24.05$; Anzahl der Anregungen $=2$; Aufnahmewinkel $=30^{\circ}$; Sichtfeld $=$ 256; Schichtebene $=$ sagittal; Matrix $=256 \times 256$; Schichtdicke $=1.3 \mathrm{~mm}$; Schichtanzahl $=$ 130-180; Aufnahmemodus = 3D). Zusätzlich wurde eine FLAIR (engl. Fluid Attenuation Inversion Recovery)-Sequenz erhoben $($ Echo-Zeit $=100 ;$ Wiederholungszeit $=6000 ;$ Anzahl der Anregungen $=2$; Inversionszeit $=2000$; Schichtebene $=$ transversal; Matrix $=179 \times 256$; Schichtdicke $=5.0 \mathrm{~mm}$; Schichtanzahl $=20$ ), die einen starken Kontrast für punktuelle Hypodensitäten im corticalen Marklager und in der subcorticalen weißen Substanz liefert (DeCoene, Hajnal \& Gatehouse, 1992; Fazekas et al., 1998) und somit zur Darstellung von White Matter Hyperintensitäten geeignet ist. Die Aufnahme der FLAIR-Sequenz wurde auf Röntgenfilm gespeichert, die $\mathrm{T}_{1}$-gewichteten Aufnahmen lagen digitalisiert vor. Die Auswertung der kernspintomographischen Daten beider Gruppen hatte folgende Zielsetzung: (1) Bestimmung des Schweregrads der in den FLAIR-Sequenz-Aufnahmen sichtbaren Hyperintensitäten, (2) Analyse der auf den $\mathrm{T}_{1}$-gewichteten Aufnahmen sichtbaren Läsionen und (3) Volumenbestimmung des Gesamtgehirns und einzelner Regions of Interest (ROIs).

\subsubsection{Auswertung der FLAIR-Sequenz-Aufnahmen}

Die FLAIR-Sequenz-Aufnahme lieferte 20 horizontale Schichtaufnahmen mit einer Dicke von $5 \mathrm{~mm}$. Die periventrikulären $(\mathrm{PVH})$ und in der deep white matter liegenden (DWMH) Hyperintensitäten wurden in vier Schweregrade eingeteilt:

Schweregrad 0: keine Hyperintensitäten identifizierbar oder lediglich geringfügige periventrikuläre Signalintensitäten im Sinne von "caps" oder "halos".

Schweregrad 1: kleine, umschriebene Hyperintensitäten (Foci) in der deep white matter.

Schweregrad 2: multiple, im Begriff zu konfluierende Foci in der deep white matter.

Schweregrad 3: größere Areale konfluierender Hyperintensitäten in der deep white matter und/oder irreguläre, sich bis in die deep white matter ziehende periventrikuläre Signalanhebungen. 
Die hier beschriebene Einteilung stellt eine Modifikation der Skala von Fazekas und Mitarbeitern (1987) dar. Auf die Unterscheidung von DWMH und PVH wurde -wie vom Autor selbst vorgeschlagen- verzichtet, da histopathologische Validierungen der im Kernspintomogramm identifizierbaren Hyperintensitäten ergeben hatten, daß den ursprünglich als leichtgradig pathologisch eingestuften periventrikulären Signalanhebungen im Sinne von "caps" oder "halos" keine pathologischen Veränderungen zugrundeliegen (Fazekas et al., 1993; Fazekas et al., 1998) (vgl. auch Abschnitt 3.2.1).

\subsubsection{Läsionsanalyse lakunärer Infarzierung}

Für die Läsionsanalyse wurden die $\mathrm{T}_{1}$-gewichteten, digitalisierten Aufnahmen aufgerufen (s.u.). Die Lokalisation der identifizierten Hyperdensitäten wurde unter Einbezug von Hirnatlanten (Mai, Assheuser \& Paxinos, 1997; Nieuwenhuys, Voogd \& Huijzen, 1991) vorgenommen:

Lakunäre Läsionen: multiple, umschriebene Hyperdensitäten in den Basalganglien und im Marklager mit einem Durchmesser $<10 \mathrm{~mm}$.

Infarkte in thalamischen Kernen: umschriebene Hyperdensitäten mit einem Durchmesser $>10 \mathrm{~mm}$ in thalamischen Kernen.

Infarkte in den Basalganglien: umschriebene Hyperdensitäten mit einem Durchmesser $>10$ mm im Nucleus Caudatus, Putamen oder Globus Pallidum.

Corticale Lakunen und Infarkte: Hyperdensitäten im Neocortex bzw. neocorticalen Marklager, unabhängig von Größe oder Lokalisation.

\subsubsection{Auswertung der Volumendatensätze: Regions of Interest (ROIs)}

Die in der Röntgendiagnostik erhobenen Volumendatensätze wurden über das klinikumseigene Intranetz auf eine Workstation überspielt. Die Auswertung erfolgte mit Hilfe der CURRY ® Software (Version 4.5; ${ }^{\mathrm{TM}}$ Neurosoft, Inc.). Die Aufnahmen wurden auf eine kontinuierliche Schichtdicke von 1,3 mm standardisiert. In einer halbautomatisierten Prozedur wurde für jeden einzelnen Datensatz eine individuelle Cortex-Schwelle festgelegt, die die Grenze zwischen Hirngewebe und Umgebung (Liquor, Knochen etc.) definiert. Das Gesamtgehirnvolumen wurde gemessen durch die schrittweise Bestimmung der dreidimensionalen Grenzen des Gehirns bis zum Erreichen der zuvor festgelegten Grauwert-Grenze. Diese schrittweise Bestimmung der Grenzen erfolgte automatisiert mittels der CURRY ® Software. 
Die Grenzen der ROIs konnten nicht automatisiert bestimmt werden, sondern mußten durch manuell vorgenommene Segmentierung festgelegt werden:

Temporallappen: Die anteriore Grenze wurde durch eine vertikale Ebene am äußersten anterioren Punkt des Temporallappens festgelegt. Auf jeder coronalen Schicht wurden die Grenzen des Temporallappens manuell segmentiert. Die Segmentierung folgte dem Verlauf der Sylvischen Fissur und trennte den temporalen Stamm von corticalem Marklager und subcorticalen Strukturen. Die posteriore Grenze wurde durch eine vertikale Ebene am äußersten posterioren Punkt des Hippocampus festgelegt. Danach wurde das Volumen innerhalb der handsegmentierten Grenzen automatisiert durch die zuvor individuell festgelegte CortexSchwelle bestimmt. Anschließend wurde der Temporallappen weiter unterteilt, um die Volumina der im Blickfang des Interesses stehenden Teilstrukturen, d.h. des temporaler Pols, des neocorticalen Temporallappens und des Hippocampus, bestimmen zu können.

Temporaler Pol: Anteriore Grenze sowie Segmentierung entsprachen der des Temporallappens (s.o.). Das posteriore Ende des Pols wurde durch eine Ebene auf derjenigen coronalen Schicht festgelegt, die die erste sichtbare Verbindung des Temporallappens mit dem basalen Vorderhirn zeigte (Limen insula) (Arnold et al., 1994). Anschließend wurde das Volumen innerhalb der handsegmentierten Grenzen automatisiert durch die zuvor individuell festgelegte Cortex-Schwelle bestimmt.

Neocorticaler Temporallappen: Zur Volumenbestimmung des neocorticalen Temporallappens wurde die zuvor durchgeführte Segmentierung des gesamten Temporallappens modfiziert, indem die limbischen Anteile des Temporallappens (Amygdala, entorhinaler Cortex, Hippocampus) von den neocorticalen manuell segmentiert wurden. Dann wurde das Volumen innerhalb der modifizierten Grenzen automatisiert durch die zuvor individuell festgelegte Cortex-Schwelle bestimmt. Vom auf diese Weise bestimmten Volumen des neocorticalen Temporallappens wurde abschließend das Volumen des temporalen Pols substrahiert.

Hippocampus: Die Umrisse des Hippocampus wurden auf sagittalen Schichten mit Markierungen versehen, die ebenfalls auf coronalen Schichten zu sehen waren. Coronal wurde die Hippocampusformation mit Teilen des Subiculums manuell vom umgebenden Gewebe segmentiert. Dabei wurde einem standardisierten Protokoll gefolgt (Pruessner et al., 2000) und zur Unterstützung auf eine neuroanatomische Schnittserie von Duvernoy zurückgegriffen (Duvernoy, 1998). Anschließend wurde das Volumen innerhalb der handsegmentierten Grenzen automatisiert durch die zuvor individuell festgelegte Cortex-Schwelle bestimmt. 


\subsubsection{Statistische ANAlyse}

Die deskriptive und inferenzstatistische Auswertung erfolgte mit Hilfe des Softwarepaketes Statistical Package for the Social Sciences (SPSS for Windows, Version 10.0). Die statistischen Berechnungen erfolgten auf Basis der Rohwerte, d.h. Zeit, erreichte Itemzahl, Anzahl Fehler bzw. Auslassungen. Bei der Seriellen Reaktionszeitaufgabe wurden die Mittelwerte der Reaktionszeiten für jeden der sechs Blöcke zur Analyse verwendet. Wenn nicht anders angegeben, betrug das $\alpha$-Niveau bei der inferenzstatistischen Analyse .05. Zusätzlich zum Signifikanzniveau wurde bei jedem Ergebnis einer hypothesenprüfenden Inferenzstatistik mit $\alpha_{\text {emp }}<0.05$ die dem Verfahren entsprechende Effektgröße ex post bestimmt. Effektgrößen liefern die Möglichkeit, Mittelwertsdifferenzen u.ä. unabhängig von der Streuung des untersuchten Parameters auf klinische Bedeutsamkeit hin zu beurteilen. Eine ex post bestimmte Effektgröße kann nach einer von Cohen (1992) vorgelegten Tabelle als klein, mittel oder groß klassifiziert werden (vgl. Bortz \& Döring, 1995, S. 568, Tab. 46).

Die Effektgröße einer Mittelwertsdifferenz im $t$-Test für unabhängige Stichproben wird berechnet nach:

$$
d=\frac{\mu_{A}-\mu_{B}}{\sigma} \text {. Es gilt } \mu_{A}=\bar{x}_{A} \text { und } \mu_{B}=\bar{x}_{B} .
$$

Die Populationsstreuung wird über die Zusammenfassung der geschätzten Streuungen in den Populationen nach der Formel

$$
\hat{\sigma}=\sqrt{\frac{\hat{\sigma}_{A}^{2}+\hat{\sigma}_{B}^{2}}{2}} \text { vorgenommen. }
$$

Die Varianz innerhalb einer Population kann durch die Multiplikation der Stichprobenvarianz s² mit dem Faktor n/(n-1) geschätzt werden (vgl. Bortz \& Döring, 1995, S. 568-569). Nach Cohen (1992) (vgl. Bortz \& Döring, 1995, S. 568, Tab. 46) können Effektgrößen im Rahmen eines $t$-Tests ab 0.2 als klein, ab 0.5 als mittel und ab 0.8 als groß klassifiziert werden.

Varianzanalytische Ergebnisse können wie folgt in Effektgrößen umgerechnet werden: SPSS for Windows liefert einen $\mathrm{Eta}^{2}$-Wert, der die Stärke der Effekte angibt. Zwischen $\eta^{2}$ und $f$ gilt folgender Zusammenhang: 


$$
f=\sqrt{\frac{\eta^{2}}{1-\eta^{2}}}(\text { vgl. Bortz \& Döring, 1995, S. 571, Gl. 9.12). }
$$

Nach Cohen (1992) (vgl. Bortz \& Döring, 1995, S. 568, Tab. 46) können Effektgrößen im Rahmen einer Varianzanalyse ab 0.1 als klein, ab 0.25 als mittel und ab 0.4 als groß klassifiziert werden.

Die Effektgröße korrelationsanalytischer Auswertungen entspricht dem Korrelationskoeffizienten $r$. Nach Cohen (1992) (vgl. Bortz \& Döring, 1995, S. 568, Tab. 46) können Effektgrößen im Rahmen einer Korrelationsanalyse ab 0.1 als klein, ab 0.3 als mittel und ab 0.5 als groß klassifiziert werden.

Hypothese H1a-d: Es wurden schlechtere neuropsychologische Testleistungen bei Patienten mit Koronarer Herzkrankheit im Vergleich zu gesunden Kontrollprobanden im (expliziten und impliziten) Gedächtnisbereich, in Tests, die differentielle Leistungen in der Informationsverarbeitung erfassen, sowie beim schlußfolgernden Denken vorhergesagt. Die Überprüfung der Mittelwertsunterschiede in allen neuropsychologischen Tests bis auf die Serielle Reaktionszeitaufgabe erfolgte mit dem $t$-Test für unabhängige Stichproben. Mußte aufgrund eines entsprechenden Ergebnisses im Levene-Test von einer Ungleichheit der Varianzen ausgegangen werden, wurde der homogenitätskorrigierte $t$-Wert nach Welch (vgl. Bortz, 1993) angegeben. Die statistische Analyse der Mittelwertsunterschiede in der Seriellen Reaktionszeitaufgabe (SRTT) erfolgte varianzanalytisch mittels eines 2 × 2-Designs mit einem Faktor Gruppe (Patienten; Kontrollprobanden) und einem Meßwiederholungsfaktor Block (Block x; Block y). Dabei gingen in die Analyse des Parameters Lerngeschwindigkeit die mittleren Reaktionszeiten der Patienten und Kontrollprobanden in Block 1 und Block 2 ein, in die Analyse des Parameters Ausmaß des impliziten Lernens die mittleren Reaktionszeiten beider Gruppen in den Blöcken 4 und 5. Es wurde in beiden Varianzanalysen ein signifikanter Haupteffekt für den Meßwiederholungsfaktor Block erwartet, der einen signifikanten Abfall der Reaktionszeiten von Block 1 zu Block 2 (Lerngeschwindigkeit) bzw. einen signifikanten Anstieg der Reaktionszeiten von Block 4 zu Block 5 (Ausmaß des impliziten Lernens) anzeigt. Im Falle eines signifikanten Haupteffekts wurde mit dem $t$-Test für abhängige Stichproben (einseitig getestet) eine Einzelanalyse für jede Gruppe vorgenommen. Signifikante Effekte für den Faktor Gruppe wurden nicht erwartet. Da eine verlangsamte Lerngeschwindigkeit sowie ein geringeres Ausmaß impliziten Lernens bei Patienten im Vergleich zu Kontrollprobanden vorhergesagt wurde, wurde in beiden Varianzanalysen ein signifikanter Interaktionseffekt erwartet. 
Problem der $\alpha$-Fehler-Kumulierung: Insgesamt wurden 30 neuropsychologische Parameter auf signifikante Mittelwertsunterschiede überprüft. Für jeden Funktionsbereich wurden einzelne Hypothesen aufgestellt, die jeweils mit mehreren Tests überprüft wurden. Im Rahmen klinisch-neuropsychologischer Fragestellungen ist die Festlegung eines schwachen Beurteilungskriteriums sinnvoll: Das Eintreffen bereits einer der Vorhersagen, d.h. die Signifikanz eines durchgeführten Tests reicht aus, um die überprüfte empirische Hypothese als zutreffend anzusehen. Daraus resultiert eine Verminderung der Strenge der Prüfung (e-Validität) unter gleichzeitiger Erhöhung der Fairness (f-Validität) (vgl. Hager, 1987, S. 77). Mit Anzahl der durchgeführten Tests pro Hypothese wächst die Wahrscheinlichkeit für den Fehler 1. Art (= $\alpha-$ Fehler) über alle durchgeführten Tests auf einen Wert an, der über den vorher festgelegten Wert $\alpha$ hinausgeht (= $\alpha$-Fehler-Kumulierung) (vgl. Hager, 1987, S. 171). Um der Gefahr der $\alpha$-Fehler-Kumulierung zu begegnen, muß das Signifikanzniveau bezüglich der Anzahl der in die Hypothesengruppe eingehenden Testverfahren adjustiert werden. Das adjustierte Signifikanzniveau hätte dann für den Bereich Informationsverarbeitung zum Beispiel $\alpha^{*}=0.05 / 15=0.003$ betragen (=Bonferoni-Adjustierung). Diese sehr konservative Adjustierung gilt vor allem für simultan durchgeführte Tests, die vollständig voneinander unabhängig sind. Obwohl Untertests einer Testbatterie so konstruiert sind, daß sie verschiedene Aspekte der Funktion messen, ist davon auszugehen, daß sie nicht vollständig voneinander unabhängig sind. In der korrigierten Adjustierung ist $\alpha^{*}=\alpha /(\mathrm{k}-\mathrm{x}+1)$ mit $\mathrm{k}=\mathrm{Anzahl}$ aller Tests und X=Anzahl signifikanter Tests (vgl. Bortz, Lienert \& Boehnke, 1990, S. 53, G1. 2.18). Obwohl es das schwache Beurteilungskriterium erlaubt, die empirische Vorhersage bei nur einem einzigen signifikanten Testergebnis als zutreffend anzusehen, wurde bei der Interpretation darauf Wert gelegt, die speziell von diesem Einzeltest überprüfte Beeinträchtigung zu analysieren und nur bedingt Rückschlüsse auf den gesamten Funktionsbereich z.B. der Informationsverarbeitung vorzunehmen.

Hypothese H2a+b: Bezüglich kernspintomographisch identifizierbarer Läsionen wurde erwartet, daß sich Patienten mit Koronarer Herzkrankheit bezüglich der Verteilung der Schweregrade der White Matter Hyperintensitäten von Kontrollprobanden unterscheiden. Desweiteren wurde vorhergesagt, daß Patienten mit Koronarer Herzkrankheit häufiger lakunäre Infarzierungen aufweisen als Kontrollprobanden. Der zur Überprüfung des Unterschieds der Häufigkeiten von Merkmalsausprägungen geltende $\chi^{2}$-Test für (allg.) k x m-Felder-Tafeln setzt voraus, daß die erwarteten Häufigkeiten nicht kleiner als fünf sind. Bei Abweichung 
sollte der auf Fisher und Yates zurückgehende exakte Test eingesetzt werden (Bortz et al., 1990).

Hypothese H2c: Es wurde vorhergesagt, daß sowohl in der Patientengruppe als auch in der Kontrollgruppe höheres Lebensalter mit höhergradigen White Matter Hyperintensitäten einhergeht. Zur Überprüfung dieser gerichteten Zusammenhangshypothese wurde die nonparametrische Spearman-Rangkorrelation eingesetzt, da es sich bei dem Parameter "Schweregrad der White Matter Hyperintensitäten" um eine ordinalskalierte Variable handelt.

Hypothese H2d: Es wurde vorhergesagt, daß sowohl in der Patienten- als auch in der Kontrollgruppe höhergradige White Matter Hyperintensitäten mit schlechteren neuropsychologischen Testleistungen einhergehen. Zur Überprüfung dieser Vohersagen müßte die nonparametrische Spearman-Rangkorrelation eingesetzt werden. Unter H2c wurden jedoch signifikante Zusammenhänge zwischen Schweregrad der White Matter Hyperintensitäten und dem Lebensalter vorhergesagt. Auch neuropsychologische Testleistungen korrelieren in hohem Maße mit dem Lebensalter. Es besteht bei den wenigsten in dieser Studie eingesetzten Testverfahren die Möglichkeit, Rohwerte in alterskorrigierte Werte zu transformieren. Aus diesem Grund wurde ein partialkorrelatives Vorgehen gewählt, das den Zusammenhang zwischen Schweregrad der White Matter Hyperintensitäten und neuropsychologischen Testleistungen in Abhängigkeit vom Lebensalter überprüft. SPSS for Windows liefert keine direkte Möglichkeit, nonparametrische Partialkorrelationen zu berechnen. Eine parametrische Partialkorrelation über rangtransformierte Daten ist jedoch identisch mit der nonparametrischen Partialkorrelation über die entsprechenden Daten (Bortz, 1993). Deshalb wurde eine Rangtransformation aller eingehenden Daten getrennt nach Gruppen durchgeführt und anschließend die parametrischen Partialkorrelationen zwischen den Rangwerten der neuropsychologischen Testleistungen und dem Schweregrad der White Matter Hyperintensitäten berechnet, bereinigt vom Einfluß der Variable Lebensalter.

Problem der $\alpha$-Fehler-Kumulierung: Im Rahmen dieser Zusammenhangshypothesen galten ebenfalls die Bedenken bezüglich der $\alpha$-Fehler-Kumulierung (s.o.). Es wurde erneut auf eine Adjustierung nach Bonferoni verzichtet. Stattdessen wurde folgendes Vorgehen gewählt: Wenn sich die Ergebnisinterpretation hauptsächlich auf die globale Hypothese beziehen soll und die Frage, welche Einzeltests signifikant wurden, lediglich eine untergeordnete Rolle spielt, kann über die Bestätigung oder Ablehnung der globalen Hypothese aufgrund der Überschreitungswahrscheinlichkeit P befunden werden. Die Überschreitungswahrscheinlich- 
keit $\mathrm{P}$ gibt an, mit welcher Wahrscheinlichkeit unter $\mathrm{k}$ Tests $\mathrm{x}$ Tests signifikant werden. Die globale $\mathrm{H}_{0}$ wird abgelehnt, wenn $\mathrm{P}<\alpha$ (vgl. Bortz et al., 1990, S. 16, Gl. 1.18 und S. 48ff). Zusätzlich wurden im vorliegenden Fall einzelne Testverfahren nach inhaltlichen Aspekten zusammengefaßt, um die Zahl der eingehenden Tests möglichst gering zu halten, (s. Abschnitt 3.3.1). Für Anzahl der eingehenden Tests $k<20$ liegen tabellierte Werte vor (s. Bortz \& Döring, 1995, S. 628ff, Tafel 1).

Hypothese H3a+b: Es wurde vorhergesagt, daß Patienten mit Koronarer Herzkrankheit im Vergleich zu gesunden Kontrollprobanden kleinere Volumina aller untersuchten temporalen Teilstrukturen, d.h. des Hippocampus, des temporalen Pols und des neocorticalen Temporallappens, aufweisen. Keine statistische Signifikanz im Mittelwertsvergleich wurde hingegen bezüglich des Gesamtgehirnvolumens erwartet. Die statistische Überprüfung aller Mittelwertsvergleiche erfolgte mit dem $t$-Test für unabhängige Stichproben. Mußte aufgrund eines entsprechendes Ergebnisses im Levene-Test von einer Ungleichheit der Varianzen ausgegangen werden, wurde der homogenitätskorrigierte $t$-Wert nach Welch (vgl. Bortz, 1993) angeben.

Problem der $\alpha$-Fehler-Kumulierung: Um der $\alpha$-Fehler-Kumulierung zu begegnen, wurde das $\alpha$-Fehler-Niveau nach der bereits erwähnten, modifizierten Bonferoni-Adjustierung korrigiert (vgl. Hypothesen H1a-d).

Hypothese H3c: Es wurden positive Zusammenhänge zwischen Hippocampusvolumen und dem Volumen des ipsilateralen temporalen Pols und neocorticalen Temporallappens vorhergesagt. Diese Vorhersage galt nur für die Patientengruppe; in der Kontrollgruppe wurden keinerlei Zusammenhänge zwischen Hippocampusvolumen und Volumina weiterer Temporallappenstrukturen angenommen. Zur Überprüfung der prognostizierten Zusammenhänge wurde die nonparametrische Spearman-Rangkorrelation eingesetzt. Obwohl Volumendaten Intervallskalenniveau aufweisen und bei morphologischen Parametern wie z.B. der Körpergröße oder eben dem Volumen des Gehirns von einer Normalverteilung ausgegangen werden kann, wurde auf eine parametrische Analyse verzichtet. Mit der Wahl des nonparametrischen Vorgehens sollte vermieden werden, daß Ausreißer zu einer Über- bzw. Unterschätzung des tatsächlichen Zusammenhangs führen.

Problem der $\alpha$-Fehler-Kumulierung: Um der $\alpha$-Fehler-Kumulierung zu begegnen, wurde in einem zu den Hypothesen $\mathbf{H 3 a}+\mathbf{b}$ analogen Vorgehen das $\alpha$-Niveau nach der modifizierten Bonferoni-Adjustierung korrigiert. 
Hypothese H3d: Es wurden in der Patientengruppe positive Zusammenhänge zwischen den Volumina temporaler Teilstrukturen und neuropsychologischen Leistungen in denjenigen Tests vorhergesagt, in denen sich im Mittelwertsvergleich schlechtere Leistungen der Patienten ergeben hatten. Zur Überprüfung der prognostizierten Zusammenhänge wurde die nonparametrische Spearman-Rangkorrelation eingesetzt.

Problem der $\alpha$-Fehler-Kumulierung: Im Rahmen dieser Zusammenhangshypothesen galten ebenfalls die Bedenken bezüglich der $\alpha$-Fehler-Kumulierung (s.o.). Es wurde erneut auf eine Adjustierung verzichtet. Stattdessen wurde die zu Hypothese H2d analoge Vorgehensweise bezüglich $\mathrm{P}<\alpha$ gewählt. Zur Reduktion der Anzahl zu überprüfender Korrelationen wurden lediglich neuropsychologische Tests, in denen Patienten signifikant schlechtere Leistungen als Kontrollprobanden gezeigt hatten, auf ihren Zusammenhang mit den Volumina temporaler Strukturen untersucht. Da für Anzahl der eingehenden Tests k>20 keine tabellierten Werte für P vorliegen, mußte die Übergangswahrscheinlichkeit berechnet werden nach:

$$
P(X \leq k)=\sum_{x=0}^{k}\left(\begin{array}{l}
n \\
x
\end{array}\right) \cdot \pi^{x} \cdot(1-\pi)^{n-x} \text { mit } \pi=\alpha=0.05 \text { (vgl. Bortz \& Döring, 1995, S. 16, Gl. }
$$
$1.18)$. 


\subsubsection{Vergleich der neuropsychologischen Testleistungen der Patienten UND KONTROLLPROBANDEN}

Die Ergebnisse der neuropsychologischen Untersuchung für Patienten und Kontrollprobanden sind in Tabelle 5 dargestellt. Mittelwertsunterschiede wurden auf statistische Signifikanz mittels $t$-Test für unabhängige Stichproben (zweiseitig getestet) bzw. varianzanalytischüberprüft. Bei einigen Testverfahren konnte die Annahme der Varianzhomogenität nicht aufrechterhalten werden. Diese Voraussetzungsverletzung ist besonders bei ungleichen Stichprobenumfängen bedeutend. Da die Umfänge der Patienten- und Kontrollstichprobe in der vorliegenden Studie ungleich sind, wurde bei Varianzinhomogenität der Welch-Test durchgeführt, der eine Homogenitätskorrektur vornimmt (Bortz, 1993). Tabelle 5 zeigt neben Prüfgröße und Signifikanzniveau bei signifikanten Mittelwertsvergleichen die ex-post bestimmte Effektgröße $d$ ( $t$-Tests) bzw. $f$ (Interaktionseffekt der Varianzanalyse) an.

Intellektuelle Fähigkeiten: Daten aller Patienten und Kontrollprobanden lagen vor. Die Leistungen der Patienten lagen in allen Untertests des HAWIE-R unter denen der Kontrollprobanden. In drei der vier Untertests erreichten die Mittelwertsunterschiede statistische Signifikanz: Allgemeines Wissen $(t[58.6]=-2.44 ; p=0.018 ; d=-0.61$ ), Gemeinsamkeitenfinden ( $t$ $[58.8]=-3.37 ; p=0.001 ; d=-0.43)$ und Bilderergänzen $(t[55.8]=-3.99 ; p=0.001 ; d=-0.60)$. Im Mosaiktest hingegen unterschieden sich die Leistungen nicht signifikant voneinander.

Gedächtnis und Lernen: Die WMS-R wurde bei allen Patienten und Kontrollprobanden durchgeführt. Die Überprüfung expliziter Gedächtnisleistungen ergab signifikant schlechtere Leistungen der Patienten in folgenden Untertests: Figural Memory $(t[59]=-2.18 ; p=0.007$; $d=-0.68)$, Verbal Paired Associates $I(t[59]=-2.26 ; p=0.028 ; d=-0.44)$, Visual Reproduction $I I(t[58.2]=-2.56 ; p=0.013 ; d=-0.30)$ sowie Logical Memory $I$ und $I I(t[59]=-3.28 ; p=0.002$; $d=-0.35$ bzw. $t$ [59] $=-3.79 ; p=0.001 ; d=-0.36$ ). Die detaillierte Analyse der Leistungen in dem Untertest Verbal Paired Associates I zeigte, daß Patienten lediglich im ersten der drei Lerndurchgänge schlechter waren $(t[58.3]=-2.32 ; p<0.05)$, in Durchgang 2 und 3 erreichten sie das Niveau der Kontrollprobanden $(p ' s>0.20)$. Daß nicht lediglich ein Einspeicherdefizit vorlag, sondern auch eine Abrufschwäche, zeigte die Kovarianzanalyse mit der Einspeicher- 
leistung im Logical Memory I als Kovariate: Der Mittelwertsunterschied zwischen Patienten und Kontrollprobanden beim verzögerten Abruf (Logical Memory II) blieb auf dem 10\%Niveau signifikant $(F[1 ; 58]=3.03 ; p=0.08)$.

Die Serielle Reaktionszeitaufgabe (SRTT) wurde lediglich von 35 der 37 Patienten durchgeführt. Bezüglich des Parameters Lerngeschwindigkeit zeigte die 2 x 2-Varianzanalyse mit dem Faktor Gruppe (Patienten; Kontrollprobanden) und der Meßwiederholung Block (Block 1; Block 2) einen signifikanten Haupteffekt für den Meßwiederholungsfaktor Block $(F[1 ; 57]=43.71 ; p=0.001)$ sowie einen signifikanten Interaktionseffekt $(F[1 ; 57]=5.03$; $p=0.029 ; f=0.29$ ). Der Haupteffekt Gruppe hingegen verfehlte das statistische Signifkanzniveau $(F[1 ; 57]=0.73 ; p=0.396)$. Zur weiteren Analyse des signifikanten Haupteffekts Block wurde mit dem $t$-Test für abhängige Stichproben (einseitig) überprüft, ob sich die Reaktionszeiten von Block 1 zu Block 2 in jeder Gruppe signifikant verringerten. Dies war in beiden Gruppen der Fall (Patienten: $t[34]=3.41 ; p<0.01$; Kontrollprobanden: $t[23]=5.81 ; p<0.01$ ). Bezüglich des Parameters Ausmaß des impliziten Lernens zeigte die 2 x 2-Varianzanalyse mit dem Faktor Gruppe (Patienten; Kontrollprobanden) und der Meßwiederholung Block (Block 4; Block 5) einen signifikanten Haupteffekt für den Meßwiederholungsfaktor Block $(F[1 ; 57]=62.04 ; p=0.001)$. Weder der Haupteffekt Gruppe $(F[1 ; 57]=1.49 ; p=0.228)$ noch der Interaktionseffekt $(F[1 ; 57]=0.58 ; p=0.449)$ erreichte statistische Signifikanz. Zur weiteren Analyse des signifikanten Haupteffekts Block wurde mit dem $t$-Test für abhängige Stichproben (einseitig getestet) überprüft, ob die Reaktionszeiten von Block 4 zu Block 5 in jeder Gruppe signifikant anstiegen. Dies war in beiden Gruppen der Fall (Patienten: $t[34]=5.39$; $p<0.01$; Kontrollprobanden: $t[23]=5.93 ; p<0.01$ ).

Aufmerksamkeit und Informationsverarbeitung: In aufmerksamkeitsabhängigen Kurzzeitgedächtnisaufgaben (WMS-R: Digit Span Forward und Backward; Visual Memory Span Forward und Backward) unterschieden sich die Leistungen der Gruppen nicht signifikant voneinander. Der WMS-R-Untertest Mental Control hingegen zeigte eine schlechtere Bearbeitungsleistung der Patienten $(t[57.1]=-2.95 ; p=0.005 ; d=-0.82)$. Der Trail-Making-Test konnte bei allen Probanden durchgeführt werden. Während Patienten und Kontrollprobanden in Teil A vergleichbare Leistungen erbrachten, deutete sich in Teil B eine höhere Reaktionszeit der Patienten an. Der Unterschied verfehlte jedoch statistische Signifikanz $(t[59]=-1.81$; $p=0.075)$.

Aus der Testbatterie zur Aufmerksamkeitsprüfung (TAP) wurden drei Untertests durchgeführt. Für Arbeitsgedächtnis und Reaktionswechsel lagen Daten von 35 Patienten und 23 
Kontrollprobanden vor. Den Untertest Geteilte Aufmerksamkeit absolvierten alle 24 Kontrollprobanden, jedoch nur 32 Patienten. In keinem der Untertests lagen signifikante Mittelwertsunterschiede der Reaktionszeiten vor. Bei der Bearbeitung der Untertests Arbeitsgedächtnis und Geteilte Aufmerksamkeit ließen die Patienten jedoch mehr Reize aus als die Kontrollprobanden. Statistische Signifikanz erreichte dieser Unterschied lediglich beim Arbeitsgedächtnis $(t[56]=3.28 ; p=0.002 ; d=0.63)$, bei der Geteilten Aufmerksamkeit lag die empirische Irrtumswahrscheinlichkeit knapp über der kritischen $(t[48.9]=1.85 ; p=0.071)$. 
Tabelle 5: Neuropsychologische Testergebnisse der Patienten und Kontrollprobanden

\begin{tabular}{|c|c|c|c|c|c|c|c|}
\hline & \multicolumn{2}{|c|}{$\begin{array}{c}\text { KHK- } \\
\text { Patienten }\end{array}$} & \multicolumn{2}{|c|}{$\begin{array}{l}\text { Kontroll- } \\
\text { probanden }\end{array}$} & \multirow[t]{2}{*}{$\begin{array}{l}\text { Prüf- } \\
\text { größe }\end{array}$} & \multirow{2}{*}{$\begin{array}{c}\begin{array}{c}\text { Signi- } \\
\text { fikanz }\end{array} \\
p\end{array}$} & \multirow[t]{2}{*}{$\begin{array}{l}\text { Effekt- } \\
\text { größe }\end{array}$} \\
\hline & MW & $(\mathrm{SD})$ & MW & $(\mathrm{SD})$ & & & \\
\hline \multicolumn{8}{|l|}{$H A W I E-R$} \\
\hline Allgemeines Wissen ${ }^{1,2}$ & 15.7 & $(4.5)$ & 18.0 & $(3.1)$ & $t^{a}[58.6]=-2.44$ & 0.018 & -0.61 \\
\hline Gemeinsamkeitenfinden ${ }^{1,2}$ & 24.1 & $(4.8)$ & 27.5 & $(3.3)$ & $t^{a}[58.8]=-3.37$ & 0.001 & -0.43 \\
\hline Bilderergänzen $n^{1,2}$ & 12.6 & $(3.3)$ & 15.1 & $(1.6)$ & $t^{a}[55.8]=-3.99$ & 0.001 & -0.60 \\
\hline Mosaiktest ${ }^{1}$ & 29.4 & $(5.8)$ & 30.2 & $(7.6)$ & $t[59]=-0.50$ & $>0.05$ & \\
\hline \multicolumn{8}{|l|}{$W M S-R$} \\
\hline Mental Control ${ }^{1,2}$ & 4.7 & $(1.2)$ & 5.5 & $(0.9)$ & $t^{a}[57.1]=-2.95$ & 0.005 & -0.82 \\
\hline Digit Span Forward ${ }^{1}$ & 6.7 & $(1.3)$ & 6.9 & $(1.4)$ & $t[59]=-0.75$ & $>0.05$ & \\
\hline Digit Span Backward ${ }^{1}$ & 5.9 & $(1.6)$ & 6.2 & $(1.6)$ & $t[59]=-0.82$ & $>0.05$ & \\
\hline Visual Memory Span F. & 8.1 & $(1.8)$ & 7.7 & $(1.0)$ & $t^{a}[56.8]=1.11$ & $>0.05$ & \\
\hline Visual Memory Span B. ${ }^{1}$ & 7.7 & $(1.8)$ & 7.2 & $(1.5)$ & $t^{a}[55.0]=1.18$ & $>0.05$ & \\
\hline Logical Memory $I^{1,2}$ & 22.7 & $(6.8)$ & 28.5 & $(6.7)$ & $t[59]=-3.28$ & 0.002 & -0.35 \\
\hline Verbal Paired Associates $I^{1,2}$ & 19.4 & $(3.4)$ & 21.0 & $(2.3)$ & $t^{a}[58.9]=-2.26$ & 0.028 & -0.44 \\
\hline Visual Paired Associates $I^{1}$ & 11.3 & $(4.6)$ & 12.7 & $(3.2)$ & $t^{a}[58.6]=-1.38$ & $>0.05$ & \\
\hline Visual Reproduction $I^{1}$ & 35.2 & $(5.4)$ & 36.9 & $(3.5)$ & $t^{a}[58.9]=-1.51$ & $>0.05$ & \\
\hline Figural Memory ${ }^{1,2}$ & 6.3 & $(1.5)$ & 7.4 & $(1.6)$ & $t[59]=-2.18$ & 0.007 & -0.68 \\
\hline Logical Memory $I I^{1,2}$ & 17.5 & $(7.3)$ & 24.9 & $(7.6)$ & $t[59]=-3.79$ & 0.001 & -0.36 \\
\hline Verbal Paired Associates $I^{1}$ & 7.3 & $(1.1)$ & 7.6 & $(0.7)$ & $t^{a}[58.3]=-1.44$ & $>0.05$ & \\
\hline Visual Paired Associates $I^{1}$ & 4.9 & $(1.8)$ & 4.9 & $(1.5)$ & $t[59]=-0.04$ & $>0.05$ & \\
\hline Visual Reproduction $I I^{1,2}$ & 30.8 & $(8.1)$ & 35.3 & $(5.5)$ & $t^{a}[58.2]=-2.56$ & 0.013 & -0.30 \\
\hline \multicolumn{8}{|l|}{$S R T T$} \\
\hline Block $1(\mathrm{~ms})-$ Block $2(\mathrm{~ms})^{3,4}$ & 49 & $(85)$ & 99 & $(84)$ & $F[1 ; 57]=5.03$ & 0.029 & 0.29 \\
\hline Block $5(m s)-$ Block $4(m s)^{3}$ & 47 & $(52)$ & 58 & $(48)$ & $F[1 ; 57]=0.58$ & $>0.05$ & \\
\hline \multicolumn{8}{|l|}{ Trail Making } \\
\hline Teil $A(s)^{1}$ & 36 & (10) & 33 & (11) & $t[59]=1.24$ & $>0.05$ & \\
\hline Teil B $(s)^{1}$ & 84 & $(26)$ & 70 & $(32)$ & $t[59]=1.81$ & $>0.05$ & \\
\hline \multicolumn{8}{|l|}{$T A P$} \\
\hline \multicolumn{8}{|l|}{ Arbeitsgedächtnis } \\
\hline Reaktionszeit $(\mathrm{ms})^{1}$ & 656 & $(172)$ & 646 & (133) & $t[56]=0.22$ & $>0.05$ & \\
\hline Auslassungen ${ }^{1,2}$ & 3.8 & $(2.3)$ & 1.8 & $(2.0)$ & $t[56]=3.28$ & 0.002 & 0.63 \\
\hline Fehler $^{1}$ & & $(3.8)$ & 5.7 & $(7.2)$ & $t^{a}[30.3]=-0.72$ & $>0.05$ & \\
\hline \multicolumn{8}{|l|}{ Geteilte Aufmerksamkeit } \\
\hline Reaktionszeit $(\mathrm{ms})^{1}$ & 718 & $(101)$ & 727 & $(68)$ & $t^{a}[53.6]=-0.40$ & $>0.05$ & \\
\hline Auslassungen $^{1}$ & 3.3 & $(3.1)$ & 2.1 & $(1.6)$ & $t^{a}[48.9]=1.85$ & $>0.05$ & \\
\hline Fehler $^{1}$ & 2.7 & $(4.5)$ & 3.0 & $(2.8)$ & $t[54]=-0.32$ & $>0.05$ & \\
\hline \multicolumn{8}{|l|}{ Reaktionswechsel } \\
\hline Reaktionszeit $(\mathrm{ms})^{1}$ & 1065 & (304) & 1117 & (343) & $t[56]=-0.61$ & $>0.05$ & \\
\hline Fehler $^{1}$ & 6.6 & $(10.0)$ & 6.8 & $(8.4)$ & $t[56]=-0.09$ & $>0.05$ & \\
\hline
\end{tabular}

Legende: MW: Mittelwert; SD: Standardabweichung; HAWIE-R: Hamburg-Wechsler-Intelligenztest für Erwachsene -revidierte Fassung-; WMS-R: Wechsler-Memory-Scale -Revised-; SRTT: Serielle Reaktionszeitaufgabe; TAP: Testbatterie zur Aufmerksamkeitsprüfung; ${ }^{1} t$-Test (zweiseitig); ${ }^{2}$ Effektgröße $d,{ }^{3}$ Interaktionseffekt der 2 x 2-Varianzanalyse mit Faktor Gruppe (Patienten; Kontrollprobanden) und Meßwiederholung Block (Block x; Block y); ${ }^{4}$ Effektgröße $f ;{ }^{a}$ Homogenitätskorrektur nach Welch 


\subsubsection{EINFLUß DES INTELLEKTUELLEN LEISTUNGSNIVEAUS AUF GEDÄCHTNIS- UND AUFMERKSAMKEITSLEISTUNGEN}

Positive Korrelationen zwischen Leistungen in Intelligenztests und anderen kognitiven Funktionstests sind in der Literatur vielfach beschrieben, so auch in der Normstichprobe der neuesten deutschen Normierung der Wechsler Memory Scale (Härtling et al., 2000). Diese Korrelationen sind nicht allein Artefakte der Testkonstruktion, sondern spiegeln den tatsächlichen funktionellen Zusammenhang zwischen intellektuellem Niveau und Lern-, Gedächtnisund Aufmerksamkeitsleistungen wieder (vgl. Lezak, 1995).

In der vorliegenden Studie zeigten Patienten in Intelligenz- wie auch Gedächtnistests signifikant schlechtere Leistungen als Kontrollprobanden. Um die Möglichkeit zu überprüfen, daß Unterschiede in anderen kognitiven Funktionsbereichen ausschließlich auf Unterschiede im intellektuellen Leistungsniveau zurückgehen, wurden signifikante Mittelwertsunterschiede auf ihre Unabhängigkeit vom intellektuellen Leistungsniveau getestet. Das allgemeine intellektuelle Leistungsvermögen wurde über die vier Untertests des HAWIE-R, die als reliable Schätzer des Gesamt-IQ gelten (Tewes, 1991), geschätzt. Die Ergebnisse der einseitig getesteten Varianzanalyse mit dem geschätzten Gesamt-IQ als Kovariate zeigten, daß vier der acht Mittelwertsunterschiede bzw. der Interaktionseffekt in der SRTT auch unabhängig vom intellektuellen Leistungsvermögen signifikant blieben: Auslassungen im Arbeitsgedächtnis (F $[1 ; 55]=6.83 ; p<0.01)$, $)$, Figural Memory $(F[1 ; 58]=4.28 ; p<0.05)$, Logical Memory I und $I I(F[1 ; 58]=3.12 ; p<0.05$ bzw. $F[1 ; 58]=5.78 ; p<0.01)$ und Lerngeschwindigkeit in der SRTT (Interaktionseffekt der 2 x 2-Kovarianzanalyse: $F[1 ; 56]=4.03 ; p<0.05$ ). Mittelwertsunterschiede in den Untertests Mental Control, Verbal Paires Associates I und Visual Reproduction II hingegen erreichten in der Kovarianzanalyse keine statistische Signifikanz.

\subsection{KERNSPINTOMOGRAPHISCHE UNTERSUCHUNG}

\subsubsection{SCHWEREgRAdE DER WHITE MATTER HYPERINTENSITÄTEN IN DER FLAIR- SEQUENZ-AUFNAHME}

Aufgrund eines technischen Problems lag von einem Patienten keine FLAIR-SequenzAufnahme vor. Alle übrigen 36 Patienten sowie alle 24 Kontrollprobanden gingen in die Auswertung ein. Der Schweregrad der Deep White Matter Hyperintensitäten (DWMH) und der periventrikulären Hyperintensitäten (PVH) wurde von Frau Professor Eva Irle und der 
Autorin selbst unabhängig voneinander bewertet. Erstere bewertete die MRT blind, d.h. ohne Kenntnis der Gruppenzugehörigkeit. Die Interraterreliabilität war für beide Parameter sehr hoch (Spearman- Rangkorrelation, zweiseitig getestet: DWMH: $r_{s}=0.97, p<0.01 ; P V H: r_{s}=$ $0.85, p<0.01)$. Bei abweichender Bewertung wurde gemeinsam eine neue Beurteilung vorgenommen. Die folgende Abbildung zeigt Beispiele für die beschriebenen Schweregrade.
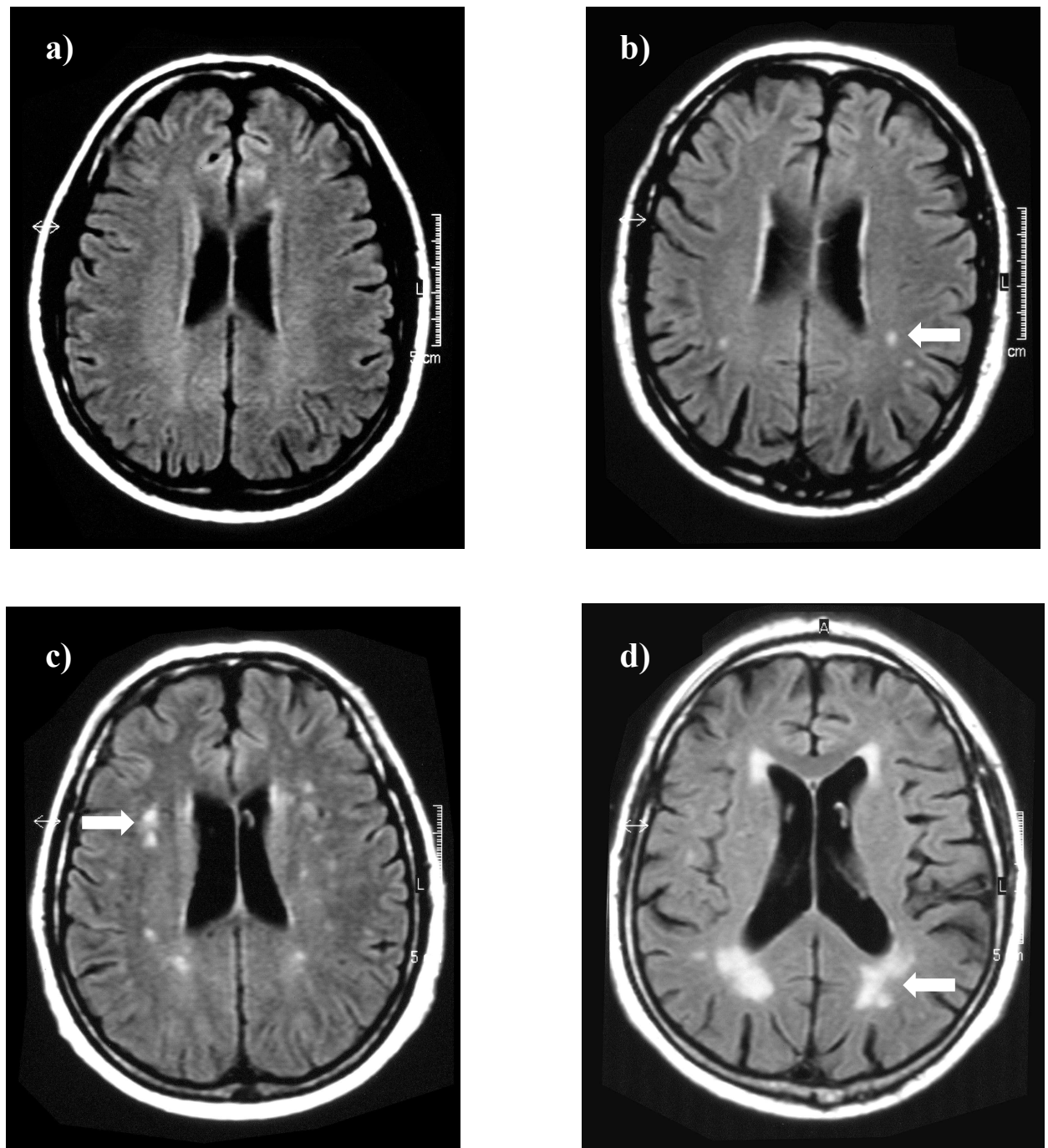

Abbildung 1: von links oben nach rechts unten: a) Schweregrad 0: keine Hyperintensitäten in der Deep White Matter und keine oder leichtgradige periventrikuläre Hyperintensitäten; b) Schweregrad 1: punktuelle Foci in der Deep White Matter (s. Pfeil); c) Schweregrad 2: leichtgradig konfluierende Foci in der Deep White Matter (s. Pfeil); d) Schweregrad 3: großflächige konfluierende Hyperintensitäten in der Deep White Matter oder/und irreguläre periventrikuläre Hyperintensitäten (s. Pfeil) 
Tabelle 6 zeigt das Ergebnis der Ratings bezüglich der $D W M H$ und $P V H$ für beide Gruppen: 16 von 36 Patienten und 11 von 24 Kontrollprobanden wiesen keine Hyperintensitäten in der $D W M$ und periventrikulär allenfalls leichtgradige periventrikuläre Hyperintensitäten im Sinne von "caps" oder "halos" auf (Schweregad 0) . Punktuelle Foci in der DWM hingegen traten bei 11 Patienten und 10 Kontrollprobanden auf (Schweregrad 1). Bei drei Patienten und zwei Kontrollprobanden begannen die Hyperintensitäten in der DWM zu konfluieren (Schweregrad 2). Schweregrad 3, d.h. großflächige konfluierende Hyperintensitäten in der DWM oder/und irreguläre periventrikuläre d.h. sich von den Ventrikelhörnern bis in die $D W M$ ziehende Hyperintensitäten wiesen sechs Patienten und ein Kontrollproband auf. Die beiden Gruppen unterschieden sich statistisch nicht in der Häufigkeitsverteilung der Schweregrade der $W M H$ (exakter Test nach Fisher und Yates).

Tabelle 6: Verteilung der Schweregrade der White Matter Hyperintensitäten in den mit der FLAIRSequenz aufgenommenen Kernspintomogrammen der Patienten und Kontrollprobanden

\begin{tabular}{lcccc}
\hline & \multicolumn{5}{c}{ Schweregrad der } \\
& \multicolumn{3}{c}{ White Matter Hyperintensitäten } \\
\cline { 2 - 5 } KHK-Patienten, $\boldsymbol{n}$ & $\mathbf{0}$ & $\mathbf{1}$ & $\mathbf{2}$ & $\mathbf{3}$ \\
\cline { 2 - 5 } & 16 & 11 & 3 & 6 \\
Kontrollprobanden, $\boldsymbol{n}$ & $(44 \%)$ & $(31 \%)$ & $(8 \%)$ & $(17 \%)$ \\
& $(46 \%)$ & $(42 \%)$ & $(8 \%)$ & $(4 \%)$ \\
\hline
\end{tabular}

Legende: n: Anzahl

\subsubsection{LAKUNÄRE INFARZIERUNG IN DER T 1 $_{1}$ GEWICHTETEN AUFNAHME}

Tabelle 7 zeigt die Häufigkeit und Verteilung der Läsionslast in der $\mathrm{T}_{1}$-gewichteten Aufnahme des Volumendatensatzes. Beide Gruppen waren in gleichem Maße von subklinisch verlaufenen Läsionen betroffen: Lediglich acht Patienten und sieben Kontrollprobanden zeigten keinerlei Läsionen. Auch in der Lokalisation der Lakunen und Infarkte unterschieden sich Patienten nicht von Kontrollprobanden. 
Tabelle 7: Häufigkeit und Lokalisation von Läsionen in der $T_{1}$-gewichteten Aufnahme bei Patienten und Kontrollprobanden

\begin{tabular}{|c|c|c|c|c|}
\hline & $\begin{array}{c}\text { KHK-Patienten } \\
\qquad(\mathrm{n}=\mathbf{3 7})\end{array}$ & $\begin{array}{c}\text { Kontrollprobanden } \\
\qquad(\mathrm{n}=\mathbf{2 4})\end{array}$ & Prüfgröße & Signifikanz \\
\hline & ja : nein & ja : nein & & $p$ \\
\hline Lakunäre Läsionen $^{1}$ & $18: 19$ & $12: 12$ & $\chi^{2}=0$ & $>0.05$ \\
\hline Infarkt im Thalamus ${ }^{2}$ & $4: 33$ & $3: 21$ & & $>0.05$ \\
\hline Infarkt in den Basalganglien ${ }^{1}$ & $9: 28$ & $5: 19$ & $\chi^{2}=0$ & $>0.05$ \\
\hline Corticale Lakunen/Infarkte ${ }^{2}$ & $4: 33$ & $1: 23$ & & $>0.05$ \\
\hline Unauffällig $^{1}$ & $8: 29$ & $7: 17$ & $\chi^{2}=0.13$ & $>0.05$ \\
\hline
\end{tabular}

Legende: ${ }^{1}$ korrigiertes $\chi^{2}$-Quadrat nach Yates (zweiseitig); ${ }^{2}$ exakter Test nach Fisher und Yates (zweiseitig)

\subsubsection{AUSWERTUNG DER VOLUMENDATENSÄTZE}

Die Auswertung der digitalisiert vorliegenden Volumendatensätze folgte der in Abschnitt 2.2.2.3 beschriebenen Vorgehensweise. Abbildung 2 zeigt die manuell durchgeführte Segmentierung des linken (seitenverkehrt im MRT) Hippocampus eines Kontrollprobanden auf jeweils einer Schicht in coronaler und sagittaler Schnittführung.
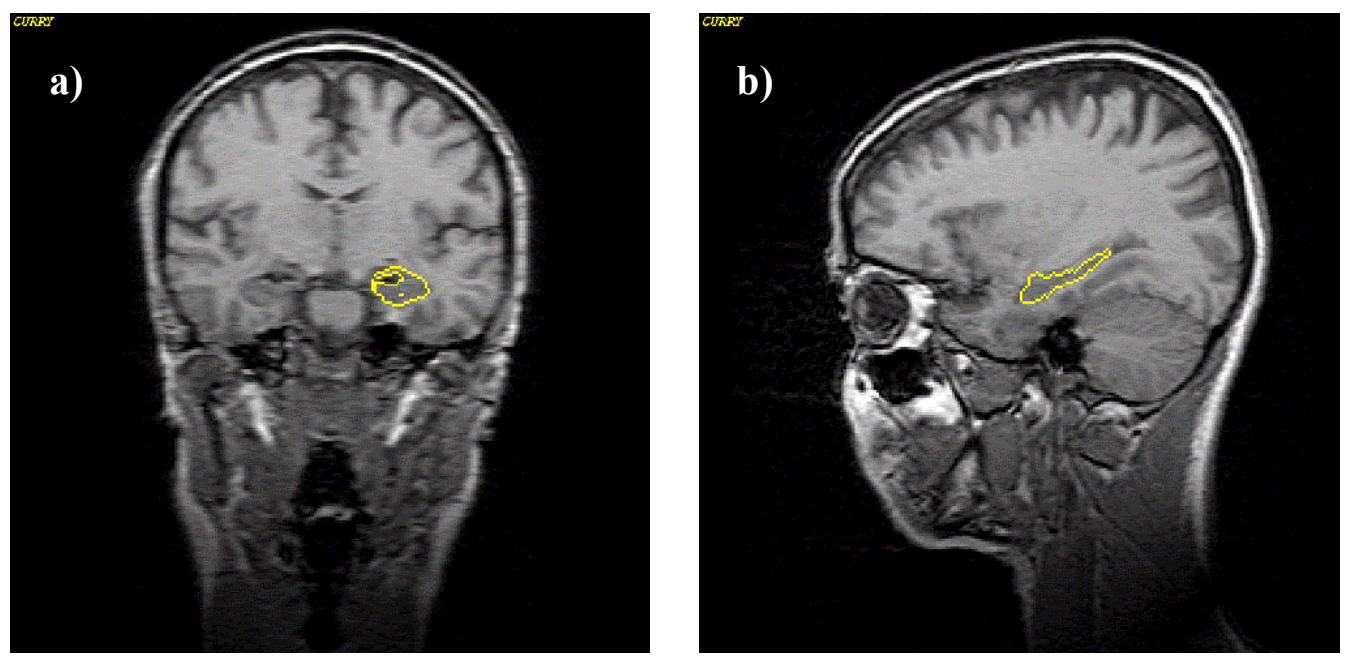

Abbildung 2: Beispiel einer manuell durchgeführten Segmentierung des Hippocampus eines Kontrollprobanden auf jeweils einer Schicht in a) coronaler und b) sagittaler Schnittführung 


\subsubsection{Mittelwertsunterschiede in den Volumina verschiedener Hirnstrukturen}

Es lagen für alle Patienten und Kontrollprobanden vollständige Datensätze vor. Die volumetrische Auswertung der 3D-Datensätze zeigte kleinere Volumina aller gemessenen Strukturen einschließlich des Gesamtgehirns bei Patienten im Vergleich zu Kontrollprobanden. Allerdings lag die Volumenreduktion des Gesamtgehirns, beider neocorticaler Temporallappen und beider temporaler Pole der Patienten lediglich zwischen 2.3-4.3\%. Anders hingegen die Volumenreduktion der Hippocampi, die für die linke Hemisphäre 10.7\% und für die rechte $9.5 \%$ betrug: Diese Mittelwertsunterschiede erreichten statistische Signifikanz (linke Hemisphäre: $t[59]=-3.62 ; p<0.01 ; d=-0.95$; rechte Hemisphäre: $t[59]=-2.88 ; p<0.01 ; d=-$ 0.75) (s. Tab. 8).

Tabelle 8: Volumina verschiedener Hirnstrukturen in der Patienten- und Kontrollgruppe

\begin{tabular}{|c|c|c|c|c|c|c|c|}
\hline \multirow[b]{2}{*}{ Volumen der Struktur in ml } & \multicolumn{2}{|c|}{$\begin{array}{c}\text { KHK- } \\
\text { Patienten } \\
(\mathbf{n}=\mathbf{3 7})\end{array}$} & \multicolumn{2}{|c|}{$\begin{array}{l}\text { Kontroll- } \\
\text { probanden } \\
(n=24)\end{array}$} & \multirow[t]{2}{*}{$\begin{array}{l}\text { Prozentualer } \\
\text { Unterschied } \\
\text { und Effekt- } \\
\text { größe }^{1}\end{array}$} & \multirow[t]{2}{*}{$\begin{array}{c}\text { Prüf- } \\
\text { größe }^{2}\end{array}$} & \multirow{2}{*}{$\begin{array}{c}\text { Signi- } \\
\text { fikanz } \\
p\end{array}$} \\
\hline & MW & (SD) & MW & (SD) & & & \\
\hline Gesamtgehirn & 1208 & $(111)$ & 1244 & $(123)$ & $-2.9(-0.31)$ & $t[59]=-1.17$ & $>0.05$ \\
\hline \multicolumn{8}{|l|}{ Neocorticaler Temporallappen } \\
\hline Links & 60.9 & $(6.9)$ & 63.1 & $(7.0)$ & $-3.5(-0.32)$ & $t[59]=-1.23$ & $>0.05$ \\
\hline Rechts & 62.3 & $(6.2)$ & 65.1 & $(7.4)$ & $-4.3(-0.46)$ & $t^{\mathrm{a}}[42.9]=-1.55$ & $>0.05$ \\
\hline \multicolumn{8}{|l|}{ Temporaler Pol } \\
\hline Links & 8.84 & $(2.10)$ & 9.08 & $(1.76)$ & $-2.6(-0.12)$ & $t[59]=-0.50$ & $>0.05$ \\
\hline Rechts & 8.55 & $(1.82)$ & 8.75 & $(2.13)$ & $-2.3(-0.10)$ & $t^{\mathrm{a}}[43.6]=-0.39$ & $>0.05$ \\
\hline \multicolumn{8}{|l|}{ Hippocampus } \\
\hline Links & 3.24 & $(0.41)$ & 3.63 & $(0.41)$ & $-10.7(-0.95)$ & $t[59]=-3.62$ & 0.001 \\
\hline Rechts & 3.32 & $(0.45)$ & 3.67 & $(0.48)$ & $-9.5(-0.75)$ & $t[59]=-2.88$ & 0.006 \\
\hline
\end{tabular}

Legende: MW: Mittelwert; SD: Standardabweichung; ${ }^{1}$ Prozentualer Unterschied: relativ zur Kontrollgruppe, Effektgröße (in Klammern); ${ }^{2}$-Test (zweiseitig); ${ }^{a}$ Homogenitätskorrektur nach Welch; 


\subsubsection{Korrelationen zwischen Volumina einzelner Temporallappenstrukturen}

Die Korrelationen zwischen Hippocampusvolumen und Volumen des temporalen Pols bzw. des neocorticalen Temporallappens wurden für die Patienten- und Kontrollgruppe getrennt berechnet. Die Ergebnisse der Spearman-Rangkorrelationen sind in Tabelle 9 abgebildet: In der Patientengruppe korrelierten die Volumina beider Hippocampi deutlich mit dem Volumen des jeweils ipsilateralen temporalen Pols (linke Hemisphäre: $r_{s}=0.423 ; p=0.035$; rechte Hemisphäre: $r_{s}=0.435 ; p=0.004$ ) bzw. des neocorticalen Temporallappens (linke Hemisphäre: $r_{s}=0.409 ; p=0.006$; rechte Hemisphäre: $\left.r_{s}=0.587 ; p=0.001\right)$. In der Kontrollgruppe hingegen waren keinerlei Zusammenhänge zwischen Hippocampusvolumina und Volumina anderer Temporallappenstrukturen zu erkennen. Einzige Ausnahme bildete die Korrelation zwischen dem Volumen des linken Hippocampus und dem Volumen des ipsilateralen neocorticalen Temporallappens $\left(r_{s}=0.378: p=0.034\right)$.

Tabelle 9: Spearman-Rangkorrelationen zwischen Volumina einzelner Temporallappenstrukturen in der Patienten- und Kontrollgruppe

\begin{tabular}{|c|c|c|c|c|c|}
\hline & \multicolumn{2}{|c|}{ Patienten $(n=37)$} & \multicolumn{3}{|c|}{ Kontrollprobanden $(n=24)$} \\
\hline & $\begin{array}{l}\text { Hippo- } \\
\text { campus li. }\end{array}$ & $\begin{array}{l}\text { Hippo- } \\
\text { campus re. }^{\text {1a }}\end{array}$ & $\begin{array}{l}\text { Hippo-- } \\
\text { campus li. }^{1 \mathrm{~b}}\end{array}$ & $\begin{array}{l}\text { Hippo- } \\
\text { campus re. }^{1 b}\end{array}$ & \\
\hline $\begin{array}{r}\text { Temporaler } \\
\text { Pol li. }\end{array}$ & $\begin{array}{l}r_{s}=0.423 \\
p=0.035\end{array}$ & - & $\begin{array}{l}r_{s}=0.096 \\
p=0.328\end{array}$ & - & $\begin{array}{l}\text { Temporaler } \\
\text { Pol li. }\end{array}$ \\
\hline $\begin{array}{r}\text { Temporaler } \\
\text { Pol re. }\end{array}$ & - & $\begin{array}{l}r_{s}=0.435 \\
p=0.004\end{array}$ & - & $\begin{array}{l}r_{s}=0.072 \\
p=0.369\end{array}$ & $\begin{array}{l}\text { Temporaler } \\
\text { Pol re. }\end{array}$ \\
\hline $\begin{array}{l}\text { Neocorticaler } \\
\text { Temporallappen li. }\end{array}$ & $\begin{array}{l}r_{s}=0.409 \\
p=0.006\end{array}$ & - & $\begin{array}{l}r_{s}=0.378 \\
p=0.034\end{array}$ & - & $\begin{array}{l}\text { Neocorticaler } \\
\text { Temporallappen li. }\end{array}$ \\
\hline $\begin{array}{l}\text { Neocorticaler } \\
\text { Temporallappen re. }\end{array}$ & - & $\begin{array}{l}r_{s}=0.587 \\
p=0.001\end{array}$ & - & $\begin{array}{l}r_{s}=0.077 \\
p=0.361\end{array}$ & $\begin{array}{l}\text { Neocorticaler } \\
\text { Temporallappen re. }\end{array}$ \\
\hline
\end{tabular}

Legende: ${ }^{1}$ Spearman-Rangkorrelation (einseitig getestet) 


\subsection{ZUSAMMENHÄNGE ZWISCHEN KERNSPINTOMOGRAPHISCHEN AUF- FÄLLIGKEITEN UND NEUROPSYCHOLOGISCHEN TESTLEISTUNGEN}

\subsubsection{ZUSAMMENHÄNGE ZWISCHEN DEM SCHWEREGRAd DER WHITE MATTER HY- PERINTENSITÄTEN UND NEUROPSYCHOLOGISCHEN TESTLEISTUNGEN}

Leistungen in einzelnen neuropsychologischen Tests wurden nach inhaltlichen Gesichtspunkten zusammengefaßt und ergaben sechs kognitive Funktionsbereiche mit insgesamt 16 Parametern:

(1) Intelligenz: Die Untertests Allgemeines Wissen (Rohwerte) und Mosaiktest (Rohwerte) aus dem HAWIE-R wurden beibehalten als Parameter des überlernten Schul- und Weltwissens und der visuokonstruktorischen Fähigkeiten. Die zuvor z-transformierten Rohwerte in den Untertests Gemeinsamkeitenfinden und Bilderergänzen wurden zu einer Variable zusammengefaßt, die die Fähigkeit zum schlußfolgernden Denken operationalisiert.

(2) Einspeicherleistungen: Als Parameter für die Leistung beim Einspeichern verbalen Materials wurden die zuvor z-transformierten Werte der WMS-R-Untertests Logical Memory I und Verbal Paired Associates I addiert. Als Parameter für die Leistung beim Einspeichern visuellen Materials wurden die zuvor z-transformierten Werte der WMS-R-Untertests Figural Memory, Visual Reproduction I und Visual Paired Associates I addiert.

(3) Abrufleistungen: Als Parameter für die Leistung beim Abruf verbalen Materials wurden die zuvor z-transformierten Werte der WMS-R-Untertests Logical Memory II und Verbal Paired Associates II addiert. Als Parameter für die Leistung beim Abruf visuellen Materials wurden die zuvor z-transformierten Werte der WMS-R-Untertests Visual Reproduction II und Visual Paired Associates II addiert.

(4) Implizites Lernen: Es wurden die bereits beschriebenen Parameter Lerngeschwindigkeit und Ausmaß des impliziten Lernens der Seriellen Reaktionszeitaufgabe verwendet, d.h. die Differenz der mittleren Reaktionszeiten von Block 1 zu Block 2 (Lerngeschwindigkeit) sowie von Block 4 zu Block 5 (Ausmaß des impliziten Lernens).

(6) Informationsverarbeitung: Es wurden Leistungen bezüglich psychomotorischer Geschwindigkeit (Trail Making Test A: Reaktionszeit), kognitiver Flexibilität (Trail Making Test B: Reaktionszeit), Arbeitsgedächtnis (TAP-Untertest Arbeitsgedächnis: Summe der z- 
transformierten Parameter Reaktionszeit, Fehler und Auslassungen), geteilter Aufmerksamkeit (TAP-Untertest Geteilte Aufmerksamkeit: Summe der z-transformierten Parameter Reaktionszeit, Fehler und Auslassungen) und Reaktionswechsel (TAP-Untertest Reaktionswechsel: Summe der z-transformierten Parameter Reaktionszeit und Fehler) unterschieden.

(6) Gedächtnisspanne: Als Parameter für die Gedächtnisspanne wurden die zuvor ztransformierten Werte der WMS-R-Untertests Digit Span Forward und Backward (verbales Material) bzw. Visual Memory Span Forward und Backward (visuelles Material) addiert.

Keinerlei statistisch bedeutsame Zusammenhänge ergaben sich zwischen Schweregrad der $W M H$ und Volumina einzelner Hirnstrukturen oder dem Gesamtgehirn in der Kontrollgruppe. In der Patientengruppe korrelierte der Schweregrad der $W M H$ lediglich negativ mit dem Volumen des rechten neocorticalen Temporallappens $\left(r_{s}=-0.280 ; p<0.05\right)$, weitere Zusammenhänge ergaben sich nicht.

Die korrelationsstatistische Analyse zeigte in der Patienten- wie auch in der Kontrollgruppe einen positiven Zusammenhang zwischen Schweregrad der WMH und Lebensalter (Patienten: $r_{s}=0.419 ; p<0.01$; Kontrollprobanden: $r_{s}=0.549 ; p<0.01$ ). Aus diesem Grund wurde ein partialkorrelatives Vorgehen gewählt, das den Zusammenhang zwischen dem Schweregrad der $W M H$ und neuropsychologischen Testleistungen in Unabhängigkeit der Variable Lebensalter überprüft.

Ergebnisse in der Patientengruppe: Der Schweregrad der $W M H$ stand mit zwei kognitiven Parametern in einem statistisch signifikanten negativen Zusammenhang: Je höhergradig die $W M H$ beurteilt wurden, desto schlechtere Leistungen ergaben sich beim schlußfolgernden Denken $\left(r_{x y \cdot z}=-0.345 ; p<0.05\right)$ und beim Abrufen verbalen Materials $\left(r_{x y z}=-0.320 ; p<\right.$ 0.05). Zwischen weiteren kognitiven Funktionen und Schweregrad der $W M H$ ergaben sich keine statistisch signifikanten Zusammenhänge. Die Überschreitungswahrscheinlichkeit, daß bei 16 durchgeführten Tests zwei signifikant sind, liegt bei $\mathrm{P}=0.043$.

Ergebnisse in der Kontrollgruppe: Zwischen dem Schweregrad der WMH ergaben sich im Bereich der Gedächtnisfunktion statistisch bedeutsame negative Zusammenhänge mit zwei Parametern: Höhergradige $W M H$ korrelierten mit schlechteren visuellen Einspeicher- sowie Abrufleistungen $\left(\mathrm{r}_{\mathrm{xy} \cdot \mathrm{z}}=-0.430 ; p<0.05\right.$ bzw. $\left.\mathrm{r}_{\mathrm{xy} \cdot \mathrm{z}}=-0.397 ; p<0.05\right)$. Ebenfalls ging eine verkürzte verbale Gedächtnisspanne mit höhergradigen $W M H$ einher $\left(\mathrm{r}_{\mathrm{xy} \cdot \mathrm{z}}=-0.407 ; p<0.05\right)$. Desweiteren korrelierte der Schweregrad der $W M H$ positiv mit einem Parameter aus dem 
Funktionsbereich Informationsverarbeitung (Reaktionswechsel: $\mathrm{r}_{\mathrm{xy} \cdot \mathrm{z}}=0.590 ; p<0.01$ ). Ein positiver Zusammenhang bedeutet hier, schlechtere Leistungen bei höhergradigen $W M H$. Die Überschreitungswahrscheinlichkeit, daß bei 16 durchgeführten Tests vier signifikant sind, liegt bei $\mathrm{P}<0.001$.

Tabelle 10: Zusammenhänge zwischen dem Schweregrad der White Matter Hyperintensitäten und kognitiven Leistungen in der Patienten- und Kontrollgruppe

\begin{tabular}{|c|c|c|c|}
\hline & & $\begin{array}{r}\text { Sch } \\
\text { White Matt }\end{array}$ & $\begin{array}{l}\text { d der } \\
\text { rintensitäten }\end{array}$ \\
\hline & & $\begin{array}{l}\text { Patienten } \\
(\mathrm{n}=36)\end{array}$ & $\begin{array}{l}\text { Kontrollprobanden } \\
\quad(\mathrm{n}=24)\end{array}$ \\
\hline & Allgemeines Wissen ${ }^{\mathrm{a}}$ & -0.281 & -0.058 \\
\hline Intelligenz & Visuokonstruktion $^{\mathrm{b}}$ & -0.107 & -0.302 \\
\hline & Schlußfolgerndes Denken $^{c}$ & $-0.345^{*}$ & -0.292 \\
\hline Einspeicher- & Verbales Material $^{\mathrm{d}}$ & -0.253 & 0.145 \\
\hline Leistungen & Visuelles Material $^{\mathrm{e}}$ & 0.017 & $-0.430 *$ \\
\hline Abruf- & Verbales Material $^{\mathrm{f}}$ & $-0.320 *$ & -0.005 \\
\hline Leistungen & Visuelles Material $^{\mathrm{g}}$ & -0.100 & $-0.397 *$ \\
\hline Implizites & Lerngeschwindigkeit $^{\mathrm{h}}$ & -0.057 & 0.140 \\
\hline Lernen & Ausmaß des impliziten Lernens ${ }^{\mathrm{i}}$ & 0.247 & -0.058 \\
\hline Gedächtnis- & Verbales Material $^{\mathrm{j}}$ & -0.174 & $-0.407^{*}$ \\
\hline spanne & Visuelles Material $^{\mathrm{k}}$ & -0.045 & -0.252 \\
\hline & Psychomotor. Geschwindigkeit ${ }^{1}$ & 0.002 & 0.176 \\
\hline Informations- & Kognitive Flexibilität ${ }^{\mathrm{m}}$ & 0.224 & 0.311 \\
\hline verarbeitung & Arbeitsgedächtnis $^{\mathrm{n}}$ & 0.271 & 0.196 \\
\hline & Geteilte Aufmerksamkeit $^{\circ}$ & 0.233 & 0.022 \\
\hline & Reaktionswechsel $^{\mathrm{p}}$ & -0.089 & $0.590 * *$ \\
\hline
\end{tabular}

Legende: ${ }^{a}$ HAWIE: Allgemeines Wissen (Rohwerte); ${ }^{b}$ HAWIE: Mosaiktest (Rohwerte); ${ }^{c}$ HAWIE: (z-Werte) Gemeinsamkeitenfinden+Bilderergänzen; ${ }^{\mathrm{d}}$ WMS: (z-Werte) Logical Memory I+Verbal Paired Associates I; ${ }^{\mathrm{e}}$ WMS: (z-Werte) Visual Reproduction I+Figural Memory+Visual Paired Associates I; ${ }^{\mathrm{f}}$ WMS: (z-Werte) Logical Memory II+Verbal Paired Associates II; ${ }^{\mathrm{g}} \mathrm{WMS}$ : (z-Werte) Visual Reproduction II+Visual Paired Associates II; ${ }^{\mathrm{h}}$ Serielle Reaktionszeitaufgabe (SRTT): Reaktionszeit Block 1-2; ${ }^{\mathrm{i}} \mathrm{SRTT}$ : Reaktionszeit Block 5-4; ${ }^{\mathrm{j}} \mathrm{WMS}$ : Digit Span (Rohwerte); ${ }^{\mathrm{k}} \mathrm{WMS}$ : Visual Memory Span (Rohwerte); ${ }^{1}$ Trail Making A (Rohwerte); ${ }^{\mathrm{m}}$ Trail Making $B$ (Rohwerte); ${ }^{\mathrm{n}} \mathrm{TAP}:$ Arbeitsgedächtnis: (z-Werte) Reaktionszeit+Fehler+Auslassungen; ${ }^{\circ} \mathrm{TAP}$ : Geteilte Aufmerksamkeit: (z-Werte) Reaktionszeit+Fehler+Auslassungen; ${ }^{\mathrm{p}} \mathrm{TAP}:$ Reaktionswechsel: (z-Werte) Reaktionszeit+Fehler; ${ }^{1}$ Partialkorrelation über Rangwerte mit Lebensalter als Einflußvariable (einseitig getestet; $\alpha=$ $0.05)$; ${ }^{*} p<0.05 ; * * p<0.01$ 


\subsubsection{ZUSAMMENHÄNGE ZWISCHEN DEN VOLUMINA TEMPORALER STRUKTUREN UND NEURopsyChologischen TeSTleistungen IN DER PATIENTENGRUPPE}

In folgenden neuropsychologischen Testverfahren hatten sich signifikant schlechtere Leistungen der Patienten mit KHK ergeben: im Intelligenzbereich in drei Untertests des HAWIE-R (Allgemeines Wissen, Gemeinsamkeitenfinden, Bilderergänzen), bei der Einspeicherung verbalen Materials in Form von Geschichten und Wortpaarassoziationen sowie visuellen Materials (WMS-R: Logical Memory I, Verbal Paired Associates I, Figural Memory), beim verzögerten Abruf der Geschichten (WMS-R: Logical Memory II), im Bereich der Informationsverarbeitung (WMS-R: Mental Control; TAP: Auslassungen im Arbeitsgedächtnis) sowie in der Geschwindigkeit, mit der eine motorische Sequenz implizit gelernt wurde (SRTT). Die nonparametrische Korrelationsanalyse (einseitig getestet) überprüfte die Zusammenhänge zwischen diesen im Vergleich zur Kontrollgruppe defizitären neuropsychologischen Testleistungen und den Volumina temporaler Strukturen.

Im Funktionsbereich Intelligenz standen schlechtere Leistungen im Allgemeinen Wissen in einem Zusammenhang mit einem geringeren Volumen des rechten temporalen Pols $\left(r_{s}=\right.$ 0.303; $p<0.05$ ), ebenso mit geringerem Volumen des linken und rechten neocorticalen Temporallappens (linke Hemisphäre: $r_{s}=0.388 ; p<0.05$; rechte Hemisphäre: $r_{s}=0.283$; $p<$ 0.05). Schlechtere Leistungen im Untertest Gemeinsamkeitenfinden waren ebenfalls mit geringerem Volumen des linken und rechten neocorticalen Temporallappens assoziiert (linke Hemisphäre: $r_{s}=0.456 ; p<0.01$; rechte Hemisphäre: $\left.r_{s}=0.419 ; p<0.01\right)$. Hingegen korrelierten schlechtere Leistungen im Bilderergänzen mit geringerem Volumen des rechten Hippocampus $\left(r_{s}=0.321 ; p<0.05\right)$.

Im Gedächtnisbereich gingen schlechtere Leistungen beim Einspeichern von Geschichten (Logical Memory I) mit geringerem Volumen des linken und rechten neocorticalen Temporallappens einher (linke Hemisphäre: $r_{s}=0.393$; $p<0.01$; rechte Hemisphäre: $r_{s}=0.404 p<$ 0.01), ebenso wie schlechtere Leistungen beim Einspeichern von Wortpaarassoziationen (Verbal Paired Associates I) (linke Hemisphäre: $r_{s}=0.321 ; p<0.05$; rechte Hemisphäre: $r_{s}=$ 0.281; $p<0.05)$. Hingegen korrelierten schlechtere Leistungen beim Einspeichern visuellen Materials (Figural Memory) mit geringerem Volumen des linken temporalen Pols $\left(r_{s}=0.273\right.$; $p<0.05$ ). Beim Abrufen von Geschichten (Logical Memory II) hingen schlechtere Leistungen mit geringerem Volumen des linken und rechten neocorticalen Temporallappens zusammen (linke Hemisphäre: $r_{s}=0.328 ; p<0.05$; rechte Hemisphäre: $r_{s}=0.379 ; p<0.01$ ). 
Beim impliziten Lernen einer motorischen Sequenz (SRTT) zeigte sich, daß eine verlangsamte Lerngeschwindigkeit mit kleinerem Volumen des linken und rechten Hippocampus assoziiert war (linke Hemisphäre: $r_{s}=0.344 ; p<0.05$; rechte Hemisphäre: $r_{s}=0.411 ; p<$ $0.01)$.

Im Bereich der Informationsverarbeitung ergaben sich signifikante Zusammenhänge zwischen einer vermehrten Anzahl von Auslassungen im TAP-Untertest Arbeitsgedächtnis und dem Volumen des linken und rechten Hippocampus (linke Hemisphäre: $r_{s}=-0.337 ; p<$ 0.05; rechte Hemisphäre: $\left.r_{s}=-0.334 ; p<0.05\right)$. Einzig der negative Zusammenhang zwischen der Leistung im WMS-R-Untertest Mental Control und dem Volumen des rechten neocorticalen Temporallappens $\left(r_{s}=-0.312 ; p<0.05\right)$ war unplausibel: Schlechtere Leistungen hingen mit größerem Volumen zusammen. Tabelle 11 zeigt die Ergebnisse der Korrelationsanalyse.

Auf die exakte Berechnung des P-Werts wurde verzichtet, da bereits der erste (und größte) Summand $\left(\begin{array}{l}60 \\ 18\end{array}\right) \cdot 0.05^{18} \cdot 0.95^{42}=4.09 .10^{-10}$ einen so kleinen Wert erreichte, daß von $\mathrm{P}<$ 0.01 ausgegangen werden konnte. 
Tabelle 11: Zusammenhänge zwischen den Volumina temporaler Strukturen und neuropsychologischen Testleistungen in der Patientengruppe

\begin{tabular}{|c|c|c|c|c|c|c|c|}
\hline & & \multicolumn{2}{|c|}{ Neocorticaler $\mathbf{T L}^{1}$} & \multicolumn{2}{|c|}{ Temporaler Pol ${ }^{1}$} & \multicolumn{2}{|c|}{ Hippocampus $^{1}$} \\
\hline & & links & rechts & links & rechts & links & rechts \\
\hline \multirow{3}{*}{ Intelligenz } & Allgemeines Wissen ${ }^{a}$ & $0.388 *$ & $0.283^{*}$ & 0.159 & $0.303 *$ & 0.109 & 0.086 \\
\hline & Gemeinsamkeitenfinden $^{a}$ & $0.456 * *$ & $0.419 *$ & 0.131 & 0.196 & 0.201 & 0.190 \\
\hline & Bilderergänzen $^{a}$ & 0.142 & 0.166 & 0.169 & 0.242 & 0.219 & $0.321 *$ \\
\hline \multirow{3}{*}{$\begin{array}{l}\text { Einspeicher- } \\
\text { leistungen }\end{array}$} & Logical Memory $I^{b}$ & $0.393 * *$ & $0.404 * *$ & 0.080 & 0.230 & -0.100 & 0.030 \\
\hline & Verbal Paired Associates $I^{b}$ & $0.321 *$ & $0.281 *$ & 0.202 & 0.044 & 0.208 & 0.180 \\
\hline & Figural Memory ${ }^{b}$ & 0.051 & 0.091 & $0.273 *$ & 0.213 & -0.026 & 0.046 \\
\hline Abrufleistungen & Logical Memory II ${ }^{b}$ & $0.328 *$ & $0.379 *$ & 0.094 & 0.141 & -0.204 & -0.069 \\
\hline $\begin{array}{l}\text { Implizites } \\
\text { Lernen }\end{array}$ & Lerngeschwindigkeit $^{c}$ & 0.119 & 0.133 & 0.094 & -0.074 & $0.344^{*}$ & $0.411 * *$ \\
\hline \multirow{2}{*}{$\begin{array}{l}\text { Informations- } \\
\text { verarbeitung }\end{array}$} & Mental Control $l^{b}$ & -0.219 & $-0.312 *$ & 0.091 & -0.080 & -0.122 & -0.115 \\
\hline & Arbeitsgedächtnis (Auslass.) ${ }^{d}$ & -0.158 & -0.196 & -0.250 & -0.074 & $-0.337 *$ & $-0.334 *$ \\
\hline
\end{tabular}

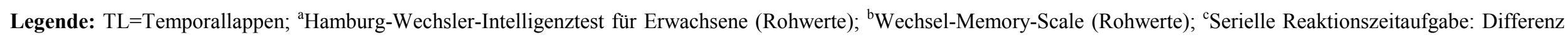
der gemittelten Reaktionszeit von Block 1 und Block $2 ;{ }^{\mathrm{d}}$ Testbatterie zur Aufmerksamkeitsprüfung; ${ }^{1}$ Spearman-Rangkorrelationen (einseitig getestet; $\alpha=0.05$ ); ${ }^{*} p<0.05 ; * *$ 


\section{$4 \quad$ DISKUSSION}

In der vorliegenden Untersuchung wurden Patienten mit Koronarer Herzkrankheit mit gesunden Kontrollprobanden bezüglich neuropsychologischer Testleistungen und kernspintomographischer Auffälligkeiten verglichen. Schlechtere kognitive Leistungen und mikroangiopathische Läsionen wie White Matter Hyperintensitäten und lakunäre Infarzierungen im Rahmen der Koronaren Herzkrankheit sind in der Literatur bereits beschrieben. Diese Ergebnisse konnten in der vorliegenden Untersuchung repliziert werden: Patienten mit Koronarer Herzkrankheit zeigten im Vergleich mit gesunden Kontrollprobanden schlechtere Leistungen im Bereich des schlußfolgernden Denkens und des explizitdeklarativen Gedächtnisses sowie eine Verlangsamung beim implizit-motorischen Lernen. Es zeigte sich, daß sich die Prävalenzraten der Patienten bezüglich der White Matter Hyperintensitäten und lakunären Infarzierungen nicht von denen der Kontrollprobanden unterschieden und in beiden Gruppen der Schweregrad der White Matter Hyperintensitäten in einem Zusammenhang mit dem Lebensalter und kognitiven Leistungen stand.

In jüngster Zeit konnten postmortem-Untersuchungen zusätzlich pathologische Veränderungen medialer Temporallappenstrukturen bei Patienten mit Koronarer Herzkrankheit nachweisen, die bisher als typisch für die Alzheimer-Demenz galten. In der hier vorgestellten Studie wurde erstmals untersucht, ob analog zu den bekannten neuropathologischen Veränderungen auch in vivo eine Reduktion des Volumens medialer Temporallappenstrukturen bei Patienten mit Koronarer Herzkrankheit nachgewiesen werden kann. Tatsächlich zeigte die quantitative Analyse der kernspintomographischen Datensätze deutlich kleinere Hippocampusvolumina in der Patientengruppe: Linksseitig betrug die Volumenreduktion 10,7\% im Vergleich zur Kontrollgruppe, rechtsseitig 9,5\%, was einem großen bzw. mittel bis großen Effekt entspricht. Andere temporale Strukturen waren nicht volumenreduziert, jedoch zeigten sich in der Patientengruppe Interkorrelationen zwischen dem Volumen des Hippocampus und den Volumina weiterer temporaler Strukturen, die in der Kontrollgruppe nicht nachweisbar waren. Die Assoziation schlechterer neuropsychologischer Testleistungen mit geringeren Volumina temporaler Strukturen kann als Beleg für die klinische Relevanz der Veränderungen im Temporallappen bei Patienten mit Koronarer Herzkrankheit interpretiert werden. 


\subsection{Allgemeine DiskUSSION}

\subsubsection{Neuropsychologische Testleistungen von Patienten mit Koronarer HERZKRANKHEIT}

Intellektuelles Leistungsniveau: Es wurde in Hypothese H1a vorhergesagt, daß Patienten mit Koronarer Herzkrankheit im Vergleich mit gesunden Kontrollprobanden schlechtere Leistungen im schlußfolgernden Denken zeigen. Die Mittelwertsvergleiche im HamburgWechsler-Intelligenztest für Erwachsene (HAWIE-R) zeigten, daß Patienten im Vergleich mit Kontrollprobanden signifikant schlechter in drei der vier Untertests abschnitten: in den Untertests Allgemeines Wissen, Gemeinsamkeitenfinden und Bilderergänzen (vgl. Tab. 5, S. 55). Kein signifikanter Unterschied ergab sich im Mosaiktest. Der Leistungsunterschied bei der Abfrage überlernten Schul- und Weltwissens (Allgemeines Wissen) verwundert zunächst in Anbetracht der Tatsache, daß sich Patienten bezüglich der erreichten Schulabschlüsse und der Ausbildungsdauer nicht von Kontrollprobanden unterschieden. Entgegensetzt zur landläufigen Meinung reagiert dieser Untertest bei jedoch bei dementiellen Syndromen sensitiv, vor allem, weil Leistungen in diesem Untertest keinem wesentlichen altersbedingten Abbau unterliegen, sondern auch im höheren Lebensalter gut erhalten bleiben (Larrabee, Largen \& Levin, 1985). Die HAWIE-R-Untertests Gemeinsamkeitenfinden und Bilderergänzen können als Operationalisierungen des schlußfolgernden Denkens aufgefaßt werden: Im Test Gemeinsamkeitenfinden muß durch Abstraktion zweier zuvor genannter Begriffe ein semantisches Konzept gebildet werden, das zu einem Oberbegriff oder einer wesentlichen Gemeinsamkeit der Begriffe führt. Beim Bilderergänzen muß ausgehend vom präsentierten Bild durch Abstraktion ein Konzept des abgebildeten Gegenstandes bzw. der abgebildeten Situation gebildet werden, das die Identifizierung des fehlenden Details ermöglicht. Die Untertests ergänzen sich und bilden zusammen eine Fähigkeit ab, die am besten als Konzeptbildung und schlußfolgerndes Denken charakterisiert werden kann. Schlechtere Leistungen im Bilderergänzen könnten auch auf ein basales Defizit auf der Ebene der visuellen Mustererkennung zurückzuführen sein. Da sich Patienten in ihren visuokonstruktorischen Leistungen im Mosaiktest jedoch nicht von Kontrollprobanden unterschieden, kann ein solches basales visuelles Defizit ausgeschlossen werden.

Nach Adjustierung des $\alpha$-Niveaus (vgl. Abschnitt 2.2.3) auf $\alpha^{*}=0.025$ verbleiben alle drei Mittelwertsvergleiche, die in diese Hypothesengruppe eingehen, signifikant. Somit kann die Hypothese H1a als bestätigt gelten. 
Explizites Gedächtnis: Es wurde in Hypothese H1b vorhergesagt, daß Patienten mit Koronarer Herzkrankheit schlechtere Lern- und Gedächtnisleistungen zeigen als gesunde Kontrollprobanden. Explizite Gedächtnisleistungen wurden mit Untertests der Wechsler-MemoryScale (WMS-R) überprüft. Mittelwertsvergleiche zeigten statistisch signifikant schlechtere Leistungen der Patienten beim Einspeichern verbalen Materials in Form von Wortpaarassoziationen oder logischen Geschichten (WMS-R: Verbal Paired Associates I; Logical Memory I). Auch das Wiedererkennen zuvor präsentierter geometrischer Muster gelang Patienten schlechter als Kontrollprobanden (WMS-R: Figural Memory). Im verzögerten, freien Abruf logischer Geschichten und visueller Stimuli zeigten sich ebenfalls Leistungsunterschiede zuungunsten der Patienten (WMS-R: Logical Memory II; Visual Reproduction II) (vgl. Tab. 5, S. 55).

Das Ergebnisprofil der Patienten weist auf Schwierigkeiten sowohl bei der Einspeicherung als auch beim Abrufen von Gedächtnisinhalten hin. Beim Lernen/ Einspeichern von Information schienen Patienten in hohem Maße von wiederholter oder zumindest längerer Informationsdarbietung zu profitieren: Wurden die zu lernenden Informationen lediglich einmal und sehr kurz präsentiert wie im Logical Memory I und Figural Memory, lagen die Leistungen der Patienten unter denen der Kontrollprobanden. War die Präsentationszeit hingegen länger (Visual Reproduction I) oder wurden die Items wiederholt dargeboten (Visual Paired Associates I; Verbal Paired Associates I), so verringerten sich die Leistungsunterschiede zwischen Patienten und Kontrollprobanden. Beim verbalen Paar-Assoziationslernen werden die richtig gelernten Paare der ersten drei Durchgänge gewertet. Patienten waren lediglich im ersten dieser drei Lerndurchgänge schlechter, in Durchgang 2 und 3 erreichten sie das Niveau der Kontrollprobanden, d.h. die Patienten profitierten in hohem Maße von der wiederholten Itemdarbietung.

Neben der defizitären Einspeicherungsleistung der Patienten zeigten die neuropsychologischen Testergebnisse zusätzlich Schwierigkeiten beim Abrufen von Gedächtnisinhalten. Der signifikante Mittelwertsunterschied in der Visual Reproduction II bei gleicher Einspeicherungsleistung im sofortigen Abruf (Visual Reproduction I) deutet auf Abrufschwierigkeiten der Patienten hin. Schlechtere Leistungen im verzögerten Abruf logischer Geschichten (Logical Memory II) könnten auf die schlechteren Testergebnisse bei der Einspeicherung (Logical Memory I) zurückgehen. Jedoch zeigte der varianzanalytische Mittelwertsvergleich der Leistungen im Logical Memory II zwischen Patienten und Kontrollprobanden, daß auch bei Kontrolle der Einspeicherungsleistung im sofortigen Abruf Leistungsunterschiede beim verzögerten Abruf signifikant blieben. Ebenfalls eine Bestätigung für schlechtere 
zögerten Abruf signifikant blieben. Ebenfalls eine Bestätigung für schlechtere Abrufleistungen der Patienten stellen die unterschiedlichen Verhältnisse der Rohwerte in Tests mit sofortigem und verzögertem Abruf dar. Die Relativierung der Rohwerte im verzögerten Abruf an den Rohwerten im sofotigen Abruf kann als ein Ausmaß der Abrufleistung interpretiert werden: Ein kleinerer Wert zeigt eine kleinere Abrufrate an, d.h. trotz gelungener Einspeicherung wurden die Inhalte unvollständig abgerufen. Die Abrufrate bei der Visual Reproduction lag in der Patientengruppe bei 87.5\%, bei der Kontrollgruppe 95.7\%. Ähnliche Unterschiede stellten sich im Logical Memory dar: Patienten wiesen eine Abrufrate von 77.1\%, Kontrollprobanden von $87.4 \%$ auf.

Insgesamt zeigten Patienten bei der Einspeicherung und beim Abrufen expliziter Gedächtnisinhalte schlechtere Leistungen als Kontrollprobanden. Drei der fünf signifikanten Mittelwertsunterschiede im Gedächtnisbereich blieben auch nach kovarianzanalytischer Kontrolle des intellektuellen Leistungsniveaus signifikant (vgl. Abschnitt 3.1.2), d.h. schlechtere Leitungen der Patienten im Bereich des expliziten Gedächtnisses sind nicht allein durch Unterschiede im intellektuellen Leistungsvermögen zu erklären. Nach Adjustierung des $\alpha$ Niveaus (vgl. Abschnitt 2.2.3) auf $\alpha^{*}=0.01$ verbleiben drei der fünf bei $\alpha=0.05$ signifikanten Mittelwertsvergleiche dieser Hypothesengruppe signifikant: Figural Memory sowie Logical Memory I und II. Die Hypothese H1b kann somit als bestätigt gelten.

Implizite Lernleistungen: Zur Objektivierung impliziter Lernleistungen wurde eine experimentelle Aufgabe zum implizit-motorischen Lernen, die Serial Reaction Time Task (SRTT), eingesetzt. Die verlangsamte oder erschwerte Konsolidierung expliziter Lerninhalte ließ sich auch bei implizit zu lernendem Material in der SRTT bestätigen: Nach drei Durchgängen, in denen die implizit zu lernende Sequenz präsentiert wurde, unterschieden sich Patienten von Kontrollprobanden nicht im Ausmaß des impliziten Lernens, operationalisiert durch die Differenz der mittleren Reaktionszeiten von Block 5 und 4. Der Vergleich der Differenz der mittleren Reaktionszeiten von Block 1 und 2 hingegen zeigte, daß Kontrollprobanden sehr viel schneller als Patienten von der Sequenz profitierten, d.h. die Sequenz sehr viel schneller als Patienten implizit lernten. Auch nach kovarianzanalytischer Kontrolle der intellektuellen Leistungsfähigkeit (vgl. Abschnitt 3.1.2) blieb der Interaktionseffekt signifikant. D.h. beim Erlernen einer impliziten motorischen Sequenz zeigte sich eine verlangsamte Lerngeschwindigkeit der Patienten. Im Ausmaß des impliziten Lernens unterschieden sie sich hingegen nicht. Hypothese H1c muß somit differenziert betrachtet werden und kann nur teilweise bestätigt werden. 
Aufmerksamkeit und Informationsverarbeitung: Es wurde in Hypothese H1d vorhergesagt, daß Patienten mit Koronarer Herzkrankheit im Vergleich mit gesunden Kontrollprobanden schlechtere Leistungen in Aufgaben zeigen, die Funktionen der Aufmerksamkeit und Informationsverarbeitung überprüfen. Lediglich zwei der insgesamt 15 Mittelwertsvergleiche konnten auf dem 5\%-Niveau signifikant schlechtere Leistungen der Patienten im Vergleich zu den Kontrollprobanden aufzeigen: im WMS-R-Untertest Mental Control und im TAP (Testbatterie zur Aufmerksamkeitsprüfung)-Untertest Arbeitsgedächtnis (Anzahl Auslassungen) (vgl. Tab. 5, S. 55). Auf dem 10\%-Niveau wurden Mittelwertsunterschiede im Trail Making Test Teil B und im TAP-Untertest Geteilte Aufmerksamkeit (Anzahl Auslassungen) signifkant. Wurde das intellektuelle Leistungsniveau kovarianzanalytisch kontrolliert, blieb lediglich der Mittelwertsunterschied im TAP-Untertest Arbeitsgedächtnis (Anzahl der Auslassungen) unter dem 5\%-Fehlerniveau. Insgesamt zeigten Patienten keine deutliche Verlangsamung oder eine durchgängig schlechtere Qualität in der Informationsverarbeitung. Somit deuteten sich schwächere Leistungen der Patienten bei Anforderungen an das Arbeitsgedächtnis und die kognitive Flexibilität an. Nach Adjustierung des $\alpha$-Niveaus (vgl. Abschnitt 2.2.3) auf $\alpha^{*}=0.004$ erreichte kein Mittelwertsvergleich innerhalb dieser Hypothesengruppe statistische Signifikanz. Hypothese H1d muß somit verworfen werden.

Schlußfolgerung: Schlechtere kognitive Leistungen von Patienten mit kardiovaskulären Risikofaktoren bzw. Koronarer Herzkrankheit sind seit längerem bekannt (Breteler, Claus, Grobbee \& Hofman, 1994; Kivipelto et al., 2001; Knopman et al., 2001; Launer et al., 1995). Besonders prominent zeigten sich Defizite im Bereich des expliziten Gedächtnisses. Beeinträchtigungen im schlußfolgernden Denken sowie psychomotorische Verlangsamung und schlechtere Aufmerksamkeitsleistungen wurden ebenfalls beschrieben, allerdings nicht konsistent (Schmidt et al., 1995; Schmidt, Fazekas, Offenbacher, Lytwyn et al., 1991). Schlechtere Aufmerksamkeitsleistungen konnten in der vorliegenden Studie nicht eindeutig nachgewiesen werden. Ergebnisse zu schlechteren Lern- und Gedächtnisleistungen bei Patienten mit Koronarer Herzkrankheit werden jedoch durch die vorliegenden Studie repliziert, die darüber hinaus eine differenzierende Aussage zur Art der mnestischen Defizite erlaubt. Die mnestische Defizite der Patienten stellen sich dar als erschwerte oder verlangsamte Konsolidierung sowohl expliziter als auch impliziter Lerninhalte sowie als Abrufschwäche bei explizit gelerntem Material. Vor allem die Verlangsamung beim impliziten motorischen Lernen zeigt einen völlig neuen Aspekt defizitärer Lernleistungen bei Patienten mit vaskulären Risikofaktoren bzw. Koronarer Herzkrankheit. Bisher nicht konsistent nachgewiesen wurden schlechtere Leistungen von Patienten mit vaskulären Risikofaktoren bzw. Koronarer Herzkrankheit bei 
exekutiven Anforderungen, z.B. beim schlußfolgernden Denken. Intellektuelle Leistungen wurden in bisherigen Untersuchungen selten gezielt untersucht, da eher globale ScreeningInstrumente mit Schwerpunkt auf Gedächtnis- und Orientierungsstörungen eingesetzt wurden (Gunning-Dixon \& Raz, 2000). In der vorliegenden Untersuchung konnten sehr eindeutig defizitäre Leistungen im schlußfolgernden Denken objektiviert werden. Darüber hinaus zeigten Patienten im Vergleich zu Kontrollprobanden einen Leistungsabfall bei der Beantwortung von Fragen zum allgemeinen Weltwissen. Es wird deutlich, daß die kognitiven Leistungseinbußen von Patienten mit vaskulären Risikofaktoren bzw. Koronarer Herzkrankheit nicht auf den expliziten Gedächtnisbereich zu begrenzen sind, sondern weitere Bereiche der intellektuellen Leistungfähigkeit sowie implizite Lernprozesse betreffen. Die klinische Relevanz der Leistungsdifferenzen zwischen Patienten und Kontrollprobanden wird durch die Effektgrößen charakterisiert: Sechs der statistisch signifikanten Mittelwertsdifferenzen zeigen einen kleinen, vier einen mittleren und eine einen großen Effekt an. Vor dem Hintergrund, daß bei keinem der Patienten vor der Studienteilnahme der Verdacht einer dementiellen Entwicklung bestand, stellen diese Mittelwertsdifferenzen selbst bei lediglich kleinen bis mittelstarken Effekten ein klinisch bedeutsames Ergebnis dar.

\subsubsection{KERNSPINTOMOGRAPHISCHE AUFFÄlligKeITEN VON PATIENTEN MIT KORO- NARER HERZKRANKHEIT}

\subsubsection{White Matter Hyperintensitäten und lakunäre Infarzierung}

$\underline{\text { Schweregrad der White Matter Hyperintensitäten und Häufigkeit lakunärer Infarzie- }}$ rung: In den Hypothesen H2a bzw. H2b wurden höhergradige White Matter Hyperintensitäten (WMH) bzw. eine höhere Prävalenzrate lakunärer Infarzierungen bei Patienten mit Koronarer Herzkrankheit im Vergleich zu gesunden Kontrollprobanden vorhergesagt. Bei 17\% der Patienten, aber nur 4\% der Kontrollprobanden wurden die White Matter Hyperintensitäten als höchstgradig (Schweregrad 3) eingestuft, konfluierten also zu größeren Arealen oder stellten sich als irreguläre periventrikuläre Läsionen dar (vgl. Abb. 1, S. 57). Dennoch wich die Verteilung der Schweregrade der $W M H$ in der Patientengruppe insgesamt nicht signifikant von der in der Kontrollgruppe ab (vgl. Tab. 6, S. 58). Bezüglich der in der $\mathrm{T}_{1}$-gewichteten Aufnahme identifizierbaren Lakunen und Infarkte zeigte sich, daß 41\% der Kontrollprobanden, hingegen nur 28\% der Patienten keine Läsionen (unabhängig von Größe und Lokalisation) aufwiesen. Insgesamt unterschieden sich Patienten und Kontrollprobanden jedoch nicht signifikant bezüglich Häufigkeit und Lokalisation der in der $\mathrm{T}_{1}$-gewichteten Aufnahme sichtbaren 
Lakunen und Infarkte (vgl. Tab. 7, S. 59). Die Hypothesen H2a und H2b müssen verworfen werden.

Zusammenhang zwischen White Matter Hyperintensitäten und Lebensalter: Es wurde in Hypothese H2c vorhergesagt, daß sowohl in der Patienten- als auch in der Kontrollgruppe höhergradige White Matter Hyperintensitäten (WMH) mit höherem Lebensalter einhergehen. Dieser Zusammenhang erreichte in beiden Gruppen statistische Signifikanz (vgl. Abschnitt 3.3.1). Die Hypothese H2c kann somit als bestätigt gelten.

Dieser sehr deutliche positive Zusammenhang zwischen Schweregrad der WMH und Lebensalter liefert eine Erklärung, warum sich keine Unterschiede zwischen Patienten und Kontrollprobanden bezüglich der Schweregrade der $W M H$ ergeben hatten. Der prominente Einfluß des Lebensalters auf die Prävalenzrate von $W M H$ konnte in zahlreichen Studien bestätigt werden (Laakso et al., 1996; Lindgren et al., 1994; Longstreth et al., 1996; Schmidt et al., 1997; Sullivan, Pary, Telang, Rifai \& Zubenko, 1990). Sullivan und Mitarbeiter kommen zu dem Schluß, daß die konsistente Assoziation von ansteigendem Lebensalter und ansteigender Prävalenz der $W M H$ nahelegt, daß sich hinter dem Lebensalter kumulativ wirkende Faktoren verbergen, die in pathogenetischer Unabhängigkeit von atherothrombotischen Infarzierungen $W M H$ verursachen (ebd.).

Eine vollständige Erklärung der Tatsache, daß in der vorliegenden Studie keine Unterschiede in der Verteilung der Schweregrade der $W M H$ zwischen Patienten und Kontrollprobanden ermittelt werden konnten, kann die vorliegende Studie nicht liefern. Aus Untersuchungen ist bekannt, daß Patienten mit vaskulären Risikofaktoren bzw. Koronarer Herzkrankheit eine erhöhte Prävalenz für WMH aufzeigen (Breteler, Swieten et al., 1994; Lindgren et al., 1994; Manolio et al., 1994; Swieten, Geykes \& Derix, 1991; Veldink et al., 1998). Es ist nicht ganz auszuschließen, daß bei den in die Untersuchung aufgenommenen Kontrollprobanden vaskuläre Risikofaktoren vorlagen, die zum Zeitpunkt der Studienteilnahme unentdeckt waren. Bluthochdruck, hoher Cholesterinspiegel oder auch beginnender Diabetes mellitus führen lange Zeit zu keinerlei Beschwerden, so daß diese Erkrankungen vermutlich häufig unterdiagnostiziert bleiben. Selbstauskunft und aktuelle Medikation stellten die zur Verfügung stehenden Informationsquellen dar. Daß also bei mehr als den fünf der 24 Kontrollprobanden, die angeben hatten, blutdrucksenkende Mittel einzunehmen, vaskuläre Risikofaktoren vorgelegen haben, ist nicht völlig auszuschließen. 
Zusammenhänge zwischen White Matter Hyperintensitäten und neuropsychologischen Testleistungen: Es wurde in Hypothese H2d vorhergesagt, daß sowohl in der Patienten- als auch in der Kontrollgruppe höhergradige White Matte Hyperintensitäten (WMH) mit schlechteren neuropsychologischen Testleistungen zusammenhängen. Sowohl in der Patienten- als auch in der Kontrollgruppe gingen schlechtere Leistungen aus dem Funktionsbereich Lernen und Gedächtnis mit höhergradigen $W M H$ einher: Patienten mit höhergradigen $W M H$ waren schlechter beim Abruf verbaler Gedächtnisinhalte, Kontrollprobanden mit höhergradigen WMH konnten schlechter visuelles Material einspeichern und abrufen. Zusätzlich ergab sich bei Patienten ein negativer Zusammenhang zwischen dem Schweregrad der $W M H$ und der intellektuellen Leistungsfähigkeit (schlußfolgerndes Denken), während bei Kontrollprobanden höhergradige $W M H$ mit schlechteren Leistungen im Bereich der Aufmerksamkeit und Informationsverarbeitung einhergingen (verbale Gedächtnisspanne; Reaktionswechsel). Alle Zusammenhänge ergaben sich in Unabhängigkeit von der Einflußvariable Lebensalter (vgl. Tab. 10, S. 64). In beiden Gruppen war die Überschreitungswahrscheinlichkeit P für die empirisch nachgewiesene Anzahl signifikanter Zusammenhänge (vgl. Abschnitt 2.3.3) kleiner 0.05. Die Hypothese H2d kann somit als bestätigt gelten.

Sehr viele Studien mit sehr unterschiedlichen Designs konnten Zusammenhänge zwischen Schweregrad der WMH und neuropsychologischen Testleistungen aufzeigen bzw. schlechtere kognitive Leistungen von Probanden mit $W M H$ im Vergleich zu Probanden ohne $W M H$ nachweisen. Die dort beschriebenen Zusammenhänge sollten sorgfältig analysiert werden: Zum einen wurden häufig Verfahren eingesetzt, die keine differentiellen neuropsychologischen Testbatterien darstellen, sondern lediglich das allgemeine kognitive Leistungsniveau erfassen und vor allem in Demenz-Studien eingesetzt werden (Austrom et al., 1990; Fukuda et al., 1990; Mirsen, Lee \& Wong, 1991; Steingart et al., 1987). Zum anderen wurden Patienten untersucht, die bereits einen beginnenden dementiellen Prozeß oder klinisch relevante Schlaganfälle in der Anamnese aufwiesen (Fukuda et al., 1990; Mirsen et al., 1991). Außerdem zeigte sich immer wieder die Wichtigkeit einer Altersadjustierung in den Studien: Nach Kontrolle des Alterseffektes fielen Zusammenhänge zwischen $W M H$ und neuropsychologischen Testleistungen häufig auf ein nicht-signifikantes Niveau zurück (Breteler, Swieten et al., 1994; Tupler, Coffey, Logue, Djang \& Fagan, 1992). Werden lediglich die Studien betrachtet, die (1) differentielle neuropsychologische Testverfahren eingesetzt, (2) ältere, neurologisch unauffällige Patienten untersucht und (3) Alterseffekte statistisch kontrolliert haben, so bleiben Zusammenhänge zwischen WMH und Gedächtnisleistungen (Breteler, Amerongen et al., 1994; Ylikoski et al., 1993), exekutiven Leistungen (Boone, Miller \& Lesser, 1992; Bre- 
teler, Amerongen et al., 1994; Junqué et al., 1990; Schmidt, Fazekas \& Kleinert, 1993) sowie Verarbeitungsgeschwindigkeit (Breteler, Amerongen et al., 1994; Ylikoski et al., 1993) bestehen. Diese Ergebnisse bestätigte auch eine Meta-Analyse von Gunning-Dixon und Raz (2000). In diese Meta-Analyse gingen 23 Studien mit gesunden, d.h. neurologisch und psychiatrisch unauffälligen Probanden mit einem durchschnittlichen Lebensalter von 69 Jahren ein. Die verschiedenen neuropsychologischen Testverfahren wurden in sieben kognitive Funktionsbereiche eingeteilt, die fast deckungsgleich mit denen der vorliegenden Untersuchung waren. Im Rahmen der Meta-Analyse war es möglich, den Alterseffekt in den eingehenden Studien zu adjustieren. Höhere $\mathrm{WMH}$-Scores waren assoziiert mit schlechteren Leistungen im sofortigen und verzögerten Abruf und in Aufgaben mit Anforderungen an Exekutivfunktionen sowie mit langsamerer oder schlechterer Informationsverarbeitung.

Die vorliegende Untersuchung kann keinen Aufschluß darüber geben, welche strukturellen Veränderungen tatsächlich Arealen kernspintomographischer Signalanhebungen zugrundeliegen. Der Krankheitswert der Signalanhebungen konnte in neuropathologischen Studien nicht vollständig aufgeklärt werden. Ergebnisse wie die der vorliegenden Studie, die zeigen, daß ausgeprägtere $W M H$ mit schlechteren Leistungen in neuropsychologischen Tests einhergehen, legen zumindest nahe, daß $W M H$ eine psychologische Relevanz haben. Auf funktioneller Ebene sind die Assoziationen zwischen $W M H$ und kognitiven Leistungen als Diskonnektionen fronto-subcorticaler sowie cortico-corticaler Verschaltungen erklärbar (Filley, 1998). Vielleicht erklären $W M H$ den altersgerechten, nicht grundsätzlich als pathologisch $\mathrm{zu}$ bewertenden Abbau bestimmter kognitiver Leistungen bei gesunden älteren Menschen. Langsamere Informationsverarbeitung, eingeschränkte kognitive Flexibilität und leichte Einspeicher- und Abrufschwierigkeiten sind benigne Erscheinungen des höheren Lebensalters (Lezak, 1995). Diese Veränderungen im kognitiven Leistungsprofil sollten nicht mit dementiellen Prozessen gleichgesetzt werden. Prospektive Studien zeigten eindeutig, daß das Vorhandensein von $W M H$ keinen prädiktiven Wert hat, ob jemand im Verlauf eine Demenz entwickelt (Austrom et al., 1990; Schmidt et al., 1999; Wolf et al., 2000).

Schlußfolgerung: Die Ergebnisse der vorliegenden Studie scheinen sich nahtlos in die vorangegangenen Untersuchungen einzufügen, werden die Zusammenhänge zwischen $W M H$ und neuropsychologischen Testleistungen innerhalb jeder Gruppe betrachtet. Werden jedoch die Ergebnisse der Mittelwertsvergleiche zwischen Patienten und Kontrollprobanden hinzugezogen (vgl. Tab. 5, S. 55), dann bleibt die Frage offen, auf welche pathophysiologische Grundlage diese Mittelwertsunterschiede zurückzuführen sind. Falls $W M H$ tatsächlich eine 
altersabhängige Erscheinung darstellen, die allenfalls zu benignen Veränderungen der kognitiven Leistungsfähigkeit älterer Menschen führen, stellt sich die Frage, ob bei Patienten mit vaskulären Risikofaktoren bzw. Koronarer Herzkrankheit ein zusätzlicher hirnorganischer Prozeß abläuft, der ursächlich für die im Vergleich zu Probanden ohne Herzerkrankung schlechteren kognitiven Leistungen verantwortlich ist. Die Ergebnisse der vorliegende Studie weisen eindeutig auf einen zusätzlichen Prozeß hin (vgl. folgenden Abschnitt).

\subsubsection{Volumenreduktion temporaler Strukturen}

Unterschiede in den Volumina temporaler Strukturen: Es wurde in Hypothese H3a bzw. H3b vorhergesagt, daß Patienten mit Koronarer Herzkrankheit im Vergleich zu gesunden Kontrollprobanden kleinere Volumina des Hippocampus bzw. des temporalen Pols und des neocorticalen Temporallappens aufweisen. Patienten zeigten geringere Volumina aller gemessener Strukturen (vgl. Tab. 8, S. 60). Jedoch lagen die Volumenunterschiede lediglich zwischen $2.3 \%$ (rechter temporaler Pol) und 4.3\% (rechter neocorticaler Temporallappen) und erreichten keine statistische Signifikanz. Hypothese H3b muß somit verworfen werden. Anders hingegen die Unterschiede im Hippocampusvolumen: Verglichen mit dem Hippocampusvolumen der Kontrollprobanden war das Volumen des linken Hippocampus bei Patienten um 10.7\% reduziert, das Volumen des rechten Hippocampus um 9.5\%. Die Volumenreduktion blieb bei einem adjustierten $\alpha$-Fehlerniveau von $\alpha^{*}=0.05$ (vgl. Abschnitt 2.3.3) statistisch signifikant und erreichte für das rechte Hippocampusvolumen eine mittlere bzw. für das linke Hippocampusvolumen eine große Effektstärke. Hypothese H3a kann somit als bestätigt gelten.

Gemeinsame Varianz der Volumina temporaler Strukturen: Es wurden in Hypothese H3c positive Zusammenhänge zwischen Hippocampusvolumen und dem Volumen des ipsilateralen temporalen Pols und des neocorticalen Temporallappens in der Patientengruppe, jedoch nicht in der Kontrollgruppe, vorhergesagt. Tatsächlich waren bei Patienten in beiden Hemisphären geringeres Hippocampusvolumen mit geringerem Volumen des temporalen Pols sowie des neocorticalen Temporallappens assoziiert. In der Kontrollgruppe ergab sich lediglich ein einziger positiver Zusammenhang, die drei übrigen Korrelationen lagen in der Größenordnung $r_{\mathrm{s}}<0.10$ (vgl. Tab. 9, S. 61). Nach Adjustierung des $\alpha$-Fehlerniveaus (vgl. Abschnitt 2.3.3) auf $\alpha^{*}=0.0125$ erreichte dieser Zusammenhang jedoch keine statistische Signifikanz. Anders hingegen in der Patientengruppe: Die Zusammenhänge zwischen den Volumina temporaler Strukturen blieben auch bei einem adjustierten $\alpha$-Niveau von $\alpha^{*}=0.05$ (vgl. 
Abschnitt 2.3.3) statistisch signifikant und zeigten mittlere bis große Effekstärken an. Hypothese H3c kann somit als bestätigt gelten.

Schlußfolgerung: Eine Reduktion des Hippocampusvolumens bei Patienten mit Koronarer Herzkrankheit nachzuweisen, stellt einen entscheidenen Schritt im Erkenntnisprozeß dar: Neuropathologische Studien konnten Alzheimer-typische Veränderungen hippocampaler Strukturen in Gehirnen nicht-dementer KHK-Patienten nachweisen (Soneira \& Scott, 1996; Sparks et al., 1990; Sparks et al., 1993; Sparks et al., 1995). Mit den vorliegenden Ergebnissen ist es erstmals gelungen, pathologische Veränderungen im medialen Temporallappen bei KHK-Patienten in vivo nachzuweisen. Neben der signifikanten Volumenreduktion des Hippocampus zeigten sich in der Patientengruppe auch gemeinsame Varianzen zwischen medialen und paralimbischen sowie neocorticalen Volumina. Ähnliche Zusammenhänge fanden auch Meguro und Kollegen bei Alzheimer-Patienten. In ihrer MRT-PET-Korrelationsstudie war das Ausmaß der Hippocampus-Atrophie mit einem Hypometabolismus im ipsilateralen assoziativen Neocortex assoziiert (Meguro et al., 2001). Die Tatsache, daß in der vorliegenen Untersuchung die gemeinsamen Varianzen zwischen Volumina temporaler Strukturen in der Kontrollgruppe nicht nachgewiesen werden konnten, spricht für einen pathologischen Prozeß im Temporallappen der KHK-Patienten. Dieser Prozeß hat bereits zu einer Volumenreduktion im Hippocampus geführt und ist im Begriff, auf paralimbische und neocorticale Strukturen überzugreifen, ohne jedoch schon soweit fortgeschritten zu sein, daß er sich kernspintomographisch als Volumenreduktion abbilden ließe. Die sequentielle Ausbreitung pathologischer Veränderungen vom medialen Temporallappen auf neocorticale Strukturen im Rahmen einer Alzheimer-Demenz ist neuropathologisch mehrfach nahegelegt worden (Braack \& Braack, 1991; Braak \& Braak, 1996; Braak et al., 1999; McGeer et al., 1990) und konnte bei Alzheimer-Patienten in späteren Krankheitsstadien auch kernspintomographisch in Volumenmessungen bestätigt werden (Barber et al., 2001; Callen et al., 2001; Convit et al., 1997; Juottonen et al., 1999). Die Ergebnisse der vorliegenden Studie zu pathologischen Veränderungen im Temporallappen bei Patienten mit KHK können als wichtiger Hinweis auf einen Zusammenhang zwischen Alzheimer und Koronarer Herzkrankheit interpretiert werden.

Zusammenhänge zwischen Volumina temporaler Strukturen und neuropsychologischen Testleistungen in der Patientengruppe: Es wurden in Hypothese H3d in der Patientengruppe positive Zusammenhänge zwischen den Volumina temporaler Strukturen und neuropsychologischen Testleistungen vorhergesagt, in denen sich im Mittelwertsvergleich schlechtere Leistungen der Patienten ergeben hatten (vgl. Tab. 5, S. 55). Diese prognostizierten Zusammen- 
hänge konnten eindeutig nachgewiesen werden: Geringeres Volumen des neocorticalen Temporallappens war deutlich mit niedrigerem intellektuellen Leistungsniveau sowie schlechteren Einspeicher- und Abrufleistungen assoziiert. Etwas weniger deutlich fanden sich diese $\mathrm{Zu}$ sammenhänge auch für den temporalen Pol. Verringertes Hippocampusvolumen war eindeutig mit verlangsamter impliziter Lernleistung und schlechterer Bearbeitungsqualität bei einer Aufgabe mit Arbeitsgedächtnisanforderungen korreliert (vgl. Tab. 11, S. 67). Von 60 berechneten Zusammenhängen wurden 18 mit überwiegend mittelstarken Effekten signifikant. Die Berechnung der Überschreitungswahrscheinlichkeit (vgl. Abschnitt 2.3.3) ergab $\mathrm{P}<0.05$. Hypothese H3d kann somit als bestätigt gelten.

Schlußfolgerung: Die vorliegende Studie kann keinen direkten Nachweis einer Verursachung schlechterer kognitiver Leistungen bei Patienten mit Koronarer Herzkrankheit durch pathologische Veränderungen temporaler Strukturen erbringen. Auswirkungen von Volumenreduktionen im Rahmen eines möglichen progredienten Abbauprozesses können nicht verglichen werden mit denen umschriebener Makroinfarkte in corticalen Regionen oder subcorticalen Kerngebieten sowie gezielt gesetzten Läsionen z.B. im Rahmen der neurochirurgischen Behandlung einer medikamentenresistenten Epilepsie (vgl. Abschnitt 1.5). In einer Studie mit Alzheimer-Patienten konnten z.B. Zusammenhänge zwischen Hippocampusatrophie und Gedächtnisdefiziten gezeigt werden (Petersen et al., 2000), in einer anderen Studie von Barber und Mitarbeitern korrelierte jedoch nicht das deutlich im Vergleich zur Kontrollgruppe reduzierte Hippocampusvolumen mit Gedächtnisleistungen, sondern vielmehr Volumina lateraler Temporallappenstrukturen (Barber et al., 2001). Dieses Ergebnis erhält Bestätigung durch den Befund einer PET-Studie von Desgranges und Kollegen: Sie konnten zeigen, daß sich Zusammenhänge zwischen Hippocampus und Gedächtnis im Verlauf der Alzheimer-Erkrankung verschieben. So korrelierte bei Patienten mit fortgeschrittener Erkrankung die Bearbeitung einer story-recall-Aufgabe (ähnlich wie Logical Memory der WMS-R) mit linken temporalen Assoziationscortices, nicht jedoch mit dem Hippocampus. Sie interpretieren diesen Shift als Zeichen eines (unzureichenden) Kompensationsmechanismus, in dem zur Bearbeitung einer Aufgabe, die normalerweise limbische Regionen aktiviert hätte, neocorticale Regionen rekrutiert werden, da die limbischen Regionen bereits in zu großem Ausmaß pathologisch verändert sind (Desgranges et al., 2002). Anhand dieser Ergebnisse wird deutlich, daß statistisch nachgewiesene Zusammenhänge zwischen nachlassender kognitiver Leistungsfähigkeit und beginnenden hirnorganischen Um- und Abbauprozessen sehr subtil zu interpretieren sind. 


\subsubsection{ALTERNATIVE ERKLÄRUNGEN FÜR SCHLECHTERE KOGNITIVE LEISTUNGEN DER Patienten}

Schlechtere neuropsychologische Testleistungen von Patienten mit KHK könnten auch auf andere Faktoren zurückgehen, die unabhängig sind von hirnorganischen Veränderungen, wie sie in der vorliegenden Studie gezeigt wurden. Zwei Einflußvariablen, die zu schlechteren kognitiven Leistungen bei KHK-Patienten führen könnten, müssen diskutiert werden: Zum einen stand vor einigen Jahren die antihypertensive Medikation bei Patienten mit KHK im Verdacht, aufgrund ihrer ZNS-Wirksamkeit zu kognitiven Leistungseinbußen zu führen. Mittlerweile konnten zahlreiche Studien diesen Verdacht ausräumen (Brooks et al., 1988; Dimsdale, Newton \& Joist, 1989; Madden et al., 1986; Maxwell, Hogan \& Ebly, 1999; Prince, Bird, Bliziard \& Mann, 1996; Rogers \& Bowman, 1990). Aufgrund dieser Datenlage ist nicht davon auszugehen, daß die Medikation der Patienten in der vorliegenden Studie einen Einfluß auf die kognitive Leistungsfähigkeit hatte. Zum anderen zeigten Studien, daß die Prävalenz einer Major Depression bei Patienten mit KHK erhöht ist und einen negativen Einfluß auf den Verlauf der Erkrankung hat (Carney et al., 1987; Glassman, 1998; Nemeroff, Mussleman \& Evans, 1998). Es ist seit langem bekannt, daß gerade bei älteren Patienten depressive Symptome zu kognitiven Leistungseinbußen führen können, die so schwerwiegend sind, daß die Depression verkannt wird und stattdessen ein dementieller Prozeß vermutet wird (McAllister, 1983). In der vorliegenden Untersuchung wurde das Beck-Depressions-Inventar (BDI) (Hautzinger et al., 1994) eingesetzt, um eine standardisierte, quantitative und etablierte Einschätzung des Ausmaß depressiver Symptomatik leisten zu können. Es ergaben sich keine signifikanten Mittelwertsunterschiede im BDI zwischen Patienten und Kontrollprobanden (vgl. Tab. 2, S. 35), und die Mittelwerte beider Gruppen lagen deutlich unterhalb der Grenze, die eine klinisch bedeutsame Depression anzeigt. Eine Betrachtung der Punktwerte der einzelnen Probanden zeigt, daß lediglich zwei der Patienten und einer der Kontrollprobanden knapp oberhalb dieser Grenze lagen. Eine Beeinflussung der kognitiven Leistungsfähigkeit durch eine depressive Symptomatik kann ausgeschlossen werden. Da die in der Literatur bekannten Alternativerklärungen kognitiver Beeinträchtigungen bei Patienten mit KHK ausgeschlossen werden können, geben die deutlichen Zusammenhänge zwischen kernspintomographischen Auffälligkeiten und neuropsychologischen Testleistungen bei den Patienten zumindest einen Hinweis auf die hirnorganische Verursachung der Leistungsunterschiede, selbst wenn die Kausalität nicht direkt bewiesen werden kann. 
Nicht ausgeräumt werden kann hingegen ein Selektionsbias innerhalb der Patientengruppe: Leider ist es in der vorliegenden Untersuchung nicht gelungen, männliche und weibliche Patienten mit Koronarer Herzkrankheit in gleichem Maße zu erheben. Auch wenn die Gründe hierfür in der Erkrankung selbst und in einer in der Untersuchung nicht beeinflußbaren diagnostischen Verzerrung liegen (vgl. Abschnitt 2.1.3), stellt der daraus resultierende Stichprobenbias eine Einschränkung der Generalisierbarkeit der Untersuchungsergebnisse dar.

\subsection{ABSCHLIEßENDE BEMERKUNGEN UND AUSBLICK}

In der vorliegenden Untersuchung wurden neuropsychologische Testleistungen und kernspintomographisch identifizierbare Auffälligkeiten bei Patienten mit Koronarer Herzkankheit erhoben und mit einer Kontrollgruppe ohne Herzerkrankungen verglichen. Die Studie replizierte den prominenten Einfluß des Lebensalters auf die Prävalenzrate von kernspintomographisch ermittelten White Matter Hyperintensitäten. Assoziationen zwischen höhergradigen White Matter Hyperintensitäten und schlechteren kognitiven Leistungen in beiden Gruppen zeigten die psychologische Relevanz dieser kernspintomographischen Auffälligkeiten. Nicht bestätigt werden konnte das erhöhte Risiko für White Matter Hyperintensitäten bei Patienten mit vaskulären Risikofaktoren bzw. Koronarer Herzkrankheit. Anders die volumetrische Auswertung der kernspintomographischen 3D-Datensätze: Patienten mit Kororarer Herzkrankheit zeigten im Vergleich zu Kontrollprobanden deutliche bilaterale Volumenreduktionen der hippocampalen Formation bei gleichem Gesamtgehirnvolumen. Trotz ausbleibender Volumenunterschiede in weiteren temporalen Teilstrukturen wie dem paralimbischen temporalen Pol und dem temporalen Neocortex weisen die für die Patienten mit Koronarer Herzkrankheit spezifischen gemeinsamen Varianzen der Volumina innerhalb des Temporallappen auf eine Ausbreitung des zugrundeliegenden pathologischen Prozesses hin. Die psychologische Relevanz der Volumenreduktion des Hippocampus sowie der pathologischen Varianz der Volumina des temporalen Pols und des neocorticalen Temporallappens zeigten die Zusammenhänge zwischen kleineren Volumina und schlechteren neuropsychologischen Testleistungen in der Patientengruppe.

Die durch volumetrische Kernspintomographie nachgewiesene Volumenreduktion des Hippocampus galt lange als struktureller Marker der Alzheimer-Demenz. Die zugrundeliegende Neuropathologie umfaßt die Formation seniler Plaques und neurofibrilläre Degeneration und wurde bereits Anfang dieses Jahrhunderts von Alzheimer beschrieben. Mittlerweile 
konnte gezeigt werden, daß weder die Volumenreduktion des Hippocampus noch die erwähnten neuropathologischen Veränderungen spezifisch für die Alzheimer-Demenz sind (Bigler et al., 2000; Laakso et al., 1996). Besonderes Interesse erregten neuropathologische Untersuchungen, die Alzheimer-typische Befunde auch bei Patienten mit vaskulären Risikofaktoren bzw. Koronarer Herzkrankheit nachweisen konnten (Soneira \& Scott, 1996; Sparks et al., 1990; Sparks et al., 1993; Sparks et al., 1995). Diese Patientengruppen galten bisher als Risikogruppen für die Entwicklung einer vaskulären Demenz.

Keiner der an der Studie teilnehmenden Patienten wies kognitive Störungen auf, die die Diagnose einer Demenz gerechtfertigt hätten. Dennoch liefern sie als Gruppe das Bild eines "mild cognitive impairment" (Flicker et al., 1991; Kluger et al., 1999). Die Diagnose des "mild cognitive impairment" weist eine hohe Prävalenz im höheren Lebensalter auf. Die Bemühungen der Forschung galten der Identifizierung von Variablen, die Probanden mit einem benignen Verlauf der MCI von Probanden unterscheiden, deren MCI sich als Prodromalsymptomatik einer Alzheimer-Demenz herausstellte. Eine zur "baseline" bereits bestehende Atrophie medialer Temporallappenstrukturen insbesondere des Hippocampus erwies sich als prognostisch besonders wertvoll. D.h., Probanden mit einem "mild cognitive impairment" und einer Volumenreduktion des Hippocampus entwickeln sehr viel häufiger eine AlzheimerDemenz als Probanden mit ähnlichem kognitiven Profil ohne gleichzeitig bestehende Hippocampusatrophie (Jack et al., 1999; Jack et al., 2000; Visser et al., 2002; Yamaguchi et al., 2002). Die Patienten der vorliegenden Studie wären in diesem Sinne als high-risk-Patienten für die Entwicklung einer Alzheimer-Demenz zu betrachten.

Ob das Vorliegen vaskulärer Risikofaktoren bzw. einer Koronaren Herzkrankheit direkt die Entwicklung einer Alzheimer-Demenz steuert, indem mikroangiopathische Prozesse die Neuropathologie auslösen (Petrovitch et al., 2000), oder sie zeitlich versetzte Manifestationen eines verborgenen Pathomechanismus sind (Sparks, Martin, Gross \& Hunsaker, 2000), bleibt zur Zeit noch ungeklärt. Genetische Prädisposition im Sinne des zur Zeit viel diskutierten Apolipoproteins E ع4-Allels scheint sowohl bei Alzheimer wie auch bei der Koronaren Herzkrankheit eine wichtige Rolle zu spielen (Kalmijn et al., 1996), ebenso wie bestimmte pathophysiologische Prozesse wie z.B. "oxidativer Streß" (Felician \& Sandson, 1999). Weitere Studien müssen die in der vorliegenden Studie aufgezeigten Zusammenhänge replizieren und eine Häufung der Variablenkombination "mild cognitive impairment + Hippocampusatrophie" bei Patienten mit Koronarer Herzkrankheit nachweisen. Wenn dann längsschnittlich gezeigt werden kann, daß bei diesen Patienten das Risiko erhöht ist, an einer Alzheimer- 
Demenz zu erkranken, müssen weitreichende Konsequenzen vor allem in der prophylaktischen oder zumindest den Krankheitsverlauf positiv beeinflussenden Pharmakotherapie gezogen werden.

In der vorliegenden Studie konnten aufgrund geringer Fallzahlen keine geschlechtsspezifischen Aussagen getroffen werden. Die Problematik der Rekrutierung weiblicher Patienten mit Koronarer Herzkrankheit wurde bereits diskutiert (siehe Abschnitt 2.1.3). Es ist davon auszugehen, daß die hier diskutierten Sachverhalte nicht einfach auf weibliche KHKPatienten übertragen werden können, sondern daß eine andere Verteilung vaskulärer Risikofaktoren und genetisch sowie hormonell bedingte Unterschiede einen großen Einfluß auf die Entwicklung einer Koronaren Herzkrankheit und damit auch auf die Zusammenhänge zwischen cerebralen Veränderungen und kognitiven Leistungen haben.

Vor dem Hintergrund der vorliegenden Ergebnisse müssen Längssschnittstudien diskutiert werden, die Patienten mit Koronarer Herzkrankheit vor und nach einer aorto-koronaren Venen-Bypass-Operation untersuchen. Bei diesen Eingriffen muß die Herz-LungenMaschine eingesetzt werden, die unter Verdacht steht, cerebrale Schäden hervorzurufen. Pathogenetisch werden verschiedene intraoperative Risiken diskutiert: diffuse cerebrale Ischämie durch Hypotension/Hypoperfusion sowie fokale cerebrale Ischämien durch Mikroemboli (Braekken, Reinvang, Russell, Brucher \& Svennevig, 1998; Harrison, 1995; Siesjö et al., 1995). Postoperativ gilt die inflammatorische Reaktion des Organismus auf die Bioinkompatibilität der Herz-Lungen-Maschine als Risikofaktor (Daniel, 1996). Verschlechterung kognitiver Leistungen von KHK-Patienten nach Bypass-Operation wurde als klinische Signifikanz der durch die Herz-Lungen-Maschine ausgelösten cerebralen Schädigung interpretiert. Diese Interpretation ist jedoch fragwürdig. Der kernspintomographische Nachweis cerebraler Schädigungen im Rahmen der Bypass-Operation blieb aus (Schmidt, Fazekas, Offenbacher et al., 1993; Sellman, Hindmarsh, Ivert \& Semb, 1992; Vik, Brubakk, Rinck \& Sellevold, 1991). Lediglich bei Patienten mit manifesten neurologischen Befunden post-OP gelang ein Nachweis neuer kernspintomographisch identifizierbarer Infarzierung (Wityk et al., 2001). Auch methodisch bleiben viele Studien fragwürdig: Untersuchungen mit prä-/post-OP-Design unterscheiden sich in dem zeitlichen Intervall, das zwischen den beiden Erhebungsterminen liegt. In einigen Fällen wurden Patienten im frühen postoperativen Verlauf, d.h. innerhalb der ersten 5-7 Tage, untersucht (Kneebone, Andrew, Baker \& Knight, 1998; O’Brien et al., 1992; Walzer, Herrmann \& Wallesch, 1997). Dieses Intervall eignet sich jedoch nicht, permanente kognitive Verschlechterungen auf der Basis eingetretener cerebraler Schädigungen zu objek- 
tivieren, sondern zeigt lediglich die globale postoperative Beeinträchtigung nach einem schweren Eingriff unter Vollnarkose an (Murkin, 1995). Andere Studien hingegen stellen tatsächliche Longitudinalstudien dar. Hier umfaßt das zeitliche prä-post-OP-Intervall mehrere Monate oder sogar Jahre (Madden et al., 1986; Mattlar, Engblom, Vesala, Vänttinen \& Knuts, 1991; McKhann et al., 1997; Newman et al., 2001; Selnes et al., 2001; Shaw et al., 1987). Kognitive Verschlechterungen von KHK-Patienten konnten gezeigt werden. Jedoch wurde entweder keine Kontrollgruppe über denselben Zeitraum beobachtet (Newman et al., 2001; Selnes et al., 2001) oder Probanden ohne Koronare Herzkrankheit wurden als Vergleichsgruppe eingesetzt (Shaw et al., 1987). Die in der vorliegenden Studie aufgezeigten pathologischen Veränderungen im medialen und neocorticalen Temporallappen und ihr Zusammenhang mit neuropsychologischen Testergebnissen bei nicht operierten Patienten mit Koronarer Herzkrankheit zeigen jedoch, daß eine Verschlechterung der kognitiven Leistungsfähigkeit auch ohne Bypass-Operation sehr wahrscheinlich ist. Deswegen können Rückschlüsse auf die Verursachung dieser Verschlechterungen nur gezogen werden, wenn Patienten mit Koronarer Herzkrankheit längsschnittlich untersucht werden, die sich einer Bypass-Operation unterziehen müssen, und mit KHK-Patienten verglichen werden, die als konservativ therapierte Kontrollprobanden fungieren. Eine erneute Einbestellung der Patienten der vorliegenden Studie nach einem Ablauf von drei Jahren ist geplant. Ergebnisse dieser zweiten Untersuchung werden dann eine Antwort liefern können auf die Frage, welches Risiko eine Bypass-Operation birgt, ob sich tatsächlich ein dementieller Prozeß hinter kernspintomographischen Auffälligkeiten und schlechteren neuropsychologischen Testleistungen verbirgt und welche Variablen den Übergang von einer leichten kognitiven Beeinträchtigung hin zu einer klinisch manifesten Demenz am zuverlässigsten vorhersagen. 


\section{LITERATURVERZEICHNIS}

Alzheimer, A. (1902). Die Seelenstörung auf arteriosklerotischer Grundlage. Allgemeine Zeitschrift für Psychiatrie, 59, 695-701.

Alzheimer, A. (1907). Über eine eigenartige Erkrankung der Hirnrinde. Allgemeine Zeitschrift für Psychiatrie, 64, 146-148.

Alzheimer, A. (1911). Über eigenartige Krankheitsfälle des späteren Alters. Zeitschrift für die gesamte Psychiatrie und Neurologie, 4, 355-356.

Arnold, S.E., Hyman, B.T. \& Hoesen, G.W. van (1994). Neuropathologic changes of the temporal pole in Alzheimer's disease and Pick's disease. Archives of Neurology, 51, 145-150.

Austrom, M.G., Thompson, R.F.J. \& Hendric, H.C. (1990). Foci of increased T2 signal intensity in MR images of healthy elderly subjects. A follow-up study. Journal of the American Geriatric Society, 38, 1133-1138.

Awad, I.A., Johnsons, P.C., Spetzler, R.F. \& Hodak, J.A. (1986). Incidental subcortical lesions identified on magnetic resonance imaging in the elderly, II: postmortem histopathological correlations. Stroke, 17, 1090-1097.

Babikian, V. \& Ropper, A.H. (1987). Binswanger's disease: a review. Stroke, 18, 2-12.

Baddeley, A.D. (1992). Working Memory. Science, 255, 556-559.

Baddeley, A.D., Baddeley, H.A., Bucks, R.S. \& Wilcock, G.K. (2001). Attentional control in Alzheimer's disease. Brain, 124, 1492-1508.

Barber, R., Ballard, C., McKeith, I.G., Gholkar, A. \& O`Brien, J.T. (2000). MRI volumetric study of dementia with Lewy bodies. Neurology, 54, 1304-1309.

Barber, R., McKeith, I.G., Ballard, C., Gholkar, A. \& O’Brien, J.T. (2001). A comparison of medial and lateral temporal lobe atrophy in dementia with Lewy Bodies and Alzheimer's disease: magnetic resonance imaging volumetric study. Dementia and Geriatric Cognitive Disorders, 12, 198-205. 
Bigler, E.D., Lowry, C.M., Anderson, C.V., Johnsons, S.C., Terry, J. \& Steed, M. (2000). Dementia, quantitative neuroimaging, and apolipoprotein E genotype. American Journal of Neuroradiology, 21, 1857-1868.

Binet, A. \& Simon, T. (1908). Le développement de l'intelligence chez les enfants. L'Année Psychologique, 14, 1-94.

Binswanger, O. (1894). Die Abgrenzung der allgemeinen progressiven Paralyse. Berliner Klinisches Wochenschreiben, 31, 1102-1105; 1137-1139; 1180-1186.

Black, F.W. (1986). Digit repetition in brain-damaged adults: clinical and theoretical implications. Journal of Clinical Psychology, 42, 770-782.

Bobinski, M., Leon, M.J. de \& Wegiel, J. (2000). The histopathologic validation of post mortem magnetic resonance imaging-determined hippocampal volume in Alzheimer's disease. Neuroscience, 95, 721-725.

Boiten, J. \& Lodder, J. (1992). Large striatocapsular infarcts: clinical presentation and comparison with lacunar and cortical infarcts. Acta Neurologica Scandinavia, 86, 298303.

Boone, K.B., Miller, B.L. \& Lesser, I.M. (1992). Neuropsychological correlates of white matter lesions in healthy elderly subjects: a threshold effect. Archives of Neurolgy, 49, 549-554.

Bornstein, R.A. \& Chelune, G.J. (1988). Factor structure of the Wechsler Memory Scale Revised. The Clinical Neuropsychologist, 2, 107-115.

Bortz, J. (1993). Statistik für Sozialwissenschaftler (4. Aufl.). Berlin: Springer.

Bortz, J. \& Döring, N. (1995). Forschungsmethoden und Evaluation. Berlin: Springer.

Bortz, J., Lienert, G.A. \& Boehnke, K. (1990). Verteilungsfreie Methoden in der Biostatistik. Berlin: Springer.

Botwinick, J., Storandt, M. \& Berg, L. (1986). A longitudinal, behavioral study of senile dementia of the Alzheimer type. Archives of Neurology, 43, 1124-1127. 
Braack, H. \& Braack, E. (1991). Neuropathological stageing of Alzheimer-related changes. Acta Neuropathologica, 82, 239-259.

Braak, E., Griffing, K., Arai, K., Bohl, J., Bratzke, H. \& Braak, H. (1999). Neuropathology of Alzheimer's disease: What is new since A. Alzheimer? European Archives of Psychiatry and Clinical Neuroscience, 249 (Suppl 3), III/14-III/22.

Braak, H. \& Braak, E. (1996). Development of Alzheimer-related neurofibrillary changes in the neocortex inversely recapitulates cortical myelogenesis. Acta Neuropathologica, 92, 197-201.

Braekken, S.K., Reinvang, I., Russell, D., Brucher, R. \& Svennevig, J.L. (1998). Association between intraoperative cerebral microembolic signals and postoperative neuropsychological deficit: comparison between patients with cardiac valve replacement and patients with coronary artery bypass grafting. Journal of Neurology, Neurosurgery, and Psychiatry, 65, 573-576.

Bray, G.A. (1978). Definition, measurement and classification of the syndromes of obesity. International Journal of Obesity, 2, 99-112.

Breteler, M.M.B., Amerongen, N.M. van, Swieten, J.C. van, Claus, J.J., Grobbee, D.E., Gijn, J. van, Hofman, A. \& Harskamp, F. van (1994). Cognitive correlates of ventricular enlargement and cerebral white matter lesions on magnetic resonance imaging. Stroke, 25, 1109-1115.

Breteler, M.M.B., Claus, J.J., Grobbee, D.E. \& Hofman, A. (1994). Cardiovascular disease and distribution of cognitive function in elderly people: the Rotterdam Study. British Medical Journal, 308, 1604-1608.

Breteler, M.M.B., Swieten, J.C. van, Bots, M.L., Grobbee, D.E., Claus, J.J., Hout, J.H.W. van den, Harskamp, F. van, Tanghe, H.J.L., Jong, P.T.V.M. de, Gijn, J. van \& Hofman, A. (1994). Cerebral white matter lesions, vascular risk factors, and cognitive function in a population-based study: the Rotterdam Study. Neurology, 44, 12461252.

Brodmann, K. (1909). Vergleichende Lokalisationslehre der Grosshirnrinde in ihren Prinzipien dargestellt auf Grund des Zellenbaus. Leipzig: J.A.Barth. 
Brooks, B., Kayser, L., Jorgensen, B., Danielsen, U., Hansen, J.E.M. \& Perrild, H. (1988). Three-week beta-adrenergic blockade does not impair or improve general intellectual function in young healthy males. Clinical Cardiology, 11, 5-8.

Butters, N., Salmon, D.P. \& Cullum, C.M. (1988). Differentiation of amnesic and demented patients with the Wechsler Memory Scale -Revisited. The Clinical Neuropsychologist, 2, 133-148.

Cabeza, R. \& Nyberg, L. (2000). Imaging cognition II: an empirical review of 275 PET and fMRI studies. Journal of Cognitive Neuroscience, 12, 1-47.

Callen, D.J.A., Black, S.E., Gao, F., Caldwell, C.B. \& Szalai, J.P. (2001). Beyond the hippocampus: MRI volumetry confirms widespread limbic atrophy in AD. Neurology, $57,1669-1674$.

Canavan, A., G., Passingham, R.E., Marsden, C.D., Quinn, N., Wyke, M. \& Polkey, C.E. (1989). Sequence ability in parkinsonians, patients with frontal lobe lesions and patients who have undergone unilateral temporal lobectomies. Neuropsychologia, 27, 787-798.

Carney, R.M., Rich, M.W., Tevelde, A., Saini, J., Clark, K. \& Jaffe, A.S. (1987). Major depressive disorder in coronary artery disease. American Journal of Cardiology, 60, 1273-1275.

Chase, T.N., Fedio, P., Foster, N.L., Brooks, R., Chiro, G. Di \& Mansi, L. (1984). Wechsler Adult Intelligence Scale performance. Cortical localization by fluorodeoxyglucose F 18-positron emission tomography. Archives of Neurology, 41, 1244-1247.

Chelune, G.J. \& Bornstein, R.A. (1988). WMS-R patterns among patients with unilateral brain lesions. The Clinical Psychologist, 2, 121-132.

Chen, P., Ratcliff, G., Belle, S.H., Cauley, J.A., DeKosky, S.T. \& Ganguli, M. (2001). Patterns of cognitive decline in presymptomatic Alzheimer disease. Archives of General Psychiatry, 58, 853-858.

Chimowitz, M.I., Awad, I.A. \& Furlan, A.J. (1989). Periventricular lesions on MRI: facts and theory. Stroke, 20, 963-967.

Cohen, J. (1992). A power primer. Psychological Bulletin, 112, 155-159. 
Convit, A., Leon, M.J. de, Tarshish, C., Sanit, S. de, Rusinek, H. \& George, A. (1997). Specific hippocampal volume reductions in individuals at risk for Alzheimer's disease. Neurobiology of Aging, 18, 131-138.

Corbett, A., Benett, H. \& Kos, S. (1994). Cognitive dysfunction after subcortical infarction. Archive of Neurology, 51, 999-1007.

Crosson, B., Greene, R.L. \& Roth, D.L. (1990). WAIS-R pattern cluster after blunt head injury. The Clinical Neuropsychologist, 4, 253-262.

Cummings, J.L. \& Huber, S.J. (1992). Visuospatial abnormalities in Parkinson's disease. In S.J. Huber \& J.L. Cummings (Eds.), Parkinson's disease: Neurobehavioral aspects. New York: Oxford University Press.

Daniel, S. (1996). Review of the multifactorial aspects of bioincompatibility in CPB. Perfusion, 11, 246-255.

DeCoene, B., Hajnal, J.V. \& Gatehouse, P. (1992). MR of the brain using brain fluid attenuated inversion recovery (FLAIR) pulse sequences. American Journal of Neuroradiology, 13, 1555-1564.

Delany, R.C., Rosen, A.J., Mattson, R.H. \& Novelly, R.A. (1980). Memory function in focal epilepsy: a comparison of nonsurgical, unilateral temporal lobe and frontal lobe samples. Cortex, 16, 103-117.

Desgranges, B., Baron, J.-C., Lavelée, C., Giffard, B., Viader, F., Sayette, V. de la \& Eustache, F. (2002). The neural substrate of episodic memory impairment in Alzheimer's disease as revealed by FDG-PET: relationship to degree of deterioration. Brain, 125, 116-1124.

Dimsdale, J.E., Newton, R.P. \& Joist, T. (1989). Neuropsychological side effects of ßblockers. Archives of Internal Medicine, 149, 514-525.

Duvernoy, H.M. (1998). The human hippocampus. Functional anatomy, vascularization, and serial sections with MRI. Berlin: Springer.

Easton, J.D. (1997). Cognitive correlates of leukoaraiosis. Cerebrovascular Diseases, 7, 129137. 
Exner, C., Koschack, J. \& Irle, I. (2002). The differential role of premotor frontal cortex and basal ganglia in motor sequence learning. Evidence from focal basal ganglia lesions. Learning and Memory, in Druck.

Exner, C., Weniger, G. \& Irle, E. (2001). Implicit and explicit memory after focal thalamic lesions. Neurology, 57, 2054-2063.

Fazekas, F., Chawluk, J.B., Alavi, A., Hurtig, H.I. \& Zimmerman, R.A. (1987). MR signal abnormalities at $1.5 \mathrm{~T}$ in Alzheimer's dementia and normal aging. American Journal of Radiology, 149, 351-356.

Fazekas, F., Kleinert, R., Offenbacher, H., Payer, F., Schmidt, R., Kleinert, G., Radner, H. \& Lechner, H. (1991). The morphologic correlate of incidental punctate white matter hyperintensities on MR images. American Journal of Neuroradiology, 12, 915921.

Fazekas, F., Kleinert, R., Offenbacher, H., Schmidt, R., Kleinert, G., Payer, F., Radner, H. \& Lechner, H. (1993). Pathologic correlates of incidental MRI white matter signal hyperintensities. Neurology, 43, 1683-1669.

Fazekas, F., Niederkorn, K., Schmidt, R., Offenbacher, H., Horner, S., Bertha, G. \& Lechner, H. (1988). White matter signal abnormalities in normal individuals: correlation with carotid ultrasonography, cerebral blood flow measurements, and cerebrovascular risk factors. Stroke, 19, 1285-1288.

Fazekas, F., Schmidt, R., Kleinert, R., Kapeller, P., Roob, G. \& Flooh, E. (1998). The spectrum of age-associated brain abnormalities: their measurement and histopathological correlates. Journal of Neural Transmisson, 53 (Suppl.), 31-9.

Feany, M.B. \& Dickson, D.W. (1996). Neurodegenerative disorders with extensive tau pathology: a comparative study and review. Annals of Neurology, 40, 139-148.

Felician, O. \& Sandson, T.A. (1999). The neurobiology and pharmacotherapy of Alzheimer's disease. Journal of Neuropsychiatry and Clinical Neuroscience, 11, 19-31.

Filley, C.M. (1998). The behavioral neurology of cerebral white matter. Neurology, 50, 15351540. 
Flicker, C., Ferris, S.H. \& Reisberg, B. (1991). Mild cognitive impairment in the elderly: predictors of dementia. Neurology, 41, 1006-1009.

Folstein, M.F., Folstein, S.E. \& McHugh, P.R. (1975). Mini-Mental-State: a practical method for grading the cognitive state of patients for the clinician. Journal of Psychiatry Research, 12, 189-199.

Fukuda, H., Kobayashi, S., Okada, K. \& Tsunematsu, T. (1990). Frontal white matter lesions and dementia in lacunar infarction. Stroke, 21, 1143-1149.

Glassman, A.H. (1998). Depression and the course of coronary artery disease. American Journal of Psychiatry, 155, 4-11.

Grädel, E. \& Schulte, H.D. (1992). Herz. In M. Allgöwer \& J.R. Siewert (Hrsg.), Chirurgie, (S. 467-497). Berlin: Springer.

Gunning-Dixon, F.M. \& Raz, N. (2000). The cognitive correlates of white matter abnormalities in normal aging. Neuropsychology, 14, 224-232.

Hager, W. (1987). Grundlagen einer Versuchsplanung zur Prüfung empirischer Hypothesen in der Psychologie. In G. Lüer (Hrsg.), Allgemeine experimentelle Psychologie (S. 43-264). Stuttgart: Gustav Fischer Verlag.

Hachinski, V.C., Potter, P. \& Merskey, H. (1987). Leuko-araiosis. Archives of Neurology, 44, 21-23.

Harrison, M.J.G. (1995). Neurologic complication of coronary artery bypass grafting: diffuse or focal ischemia? Annals of Thoracic Surgery, 59, 1356-1358.

Härtling, C., Markowitsch, H.J., Neufeld, H., Calabrese, P., Deisinger, K. \& Kessler, J. (2000). Wechsler-Gedächtnis-Test-Revidierte Fassung: Testmanual. Bern: Hans Huber.

Hautzinger, M., Bailer, M., Worall, H. \& Keller, F. (1994). Beck-Depressions-Inventar. Bern: Hans Huber.

Hébert, R., Lindsay, J., Verreault, R., Rockwood, K., Hill, G. \& Dubois, M.-F. (2000). Vascular dementia: incidence and risk factors in the Canadian Study of Health and Aging. Stroke, 31, 1487-1493. 
Hebb, D.O. \& Penfield, W. (1940). Human behavior after extensive bilateral removals from the frontal lobes. Archives of Neurology and Psychiatry, 44, 421-438.

Hirono, N., Yasuda, M., Tanimukai, S., Kitagaki, H. \& Mori, E. (2000). Effect of the apolipoprotein E epsilon4 allele on white matter hyperintensities in dementia. Stroke, $31,1263-1268$.

Hofman, A., Ott, A., Breteler, M.M., Bots, M.L., Slooter, A.J., Harskamp, F. van, Duijn, C.N. van, Broeckhoven, C. Van \& Grobbee, D.E. (1997). Atherosclerosis, apolipoprotein E, and prevalence of dementia and Alzheimer's disease in the Rotterdam Study. Lancet, 349, 151-154.

Jack, C.R., Petersen, R.C., Xu, Y.C., O’Brien, P.C., Smith, G.E., Ivnik, R.J., Boeve, B.F., Waring, S.C., Tangalos, E.G. \& Kokmen, E. (1999). Prediction of AD with MRIbased hippocampal volume in mild cognitive impairment. Neurology, 52, 13971403.

Jack, C.R.J., Petersen, R.C., Xu, Y., O’Brien, P.C., Smith, G.E., Ivnik, R.J., Boeve, B.F., Tangalos, E.G. \& Kokmen, E. (2000). Rates of hippocampal atrophy correlate with change in clinical status in aging and AD. Neurology, 55, 484-489.

Jacobs, D., Tröster, A.I. \& Butters, N. (1990). Intrusion errors on the Visual Reproduction Test of the Wechsler Memory Scale and the Wechsler Memory Scale -Revised: an analysis of demented and amnesic patients. The Clinical Neuropsychologist, 4, 177-191.

Johanson, A.M., Gustafson, L. \& Risberg, J. (1986). Behavioural observations during performance of the WAIS Block Design Test related to abnormalities of regional cerebral blood flow in organic dementia. Journal of Clinical and Experimental Neuropsychology, 8, 201-209.

Jong, P. \& Sternberg, L. (1998). Assessing coronary artery disease in women: How useful is coronary angiography? Medscape Womens Health, 3, 1.

Junqué, C., Pujol, J., Vendrell, P., Bruna, O., Jódar, M., Ribas, J., Vinas, J., Capdevila, A. \& Marti-Vilata, J.L. (1990). Leuko-araiosis on magnetic resonance imaging and speed of mental processing. Archives of Neurology, 47, 151-156. 
Juottonen, K., Laakso, M.P., Partanen, K. \& Soininen, H. (1999). Comparative MR analysis of the entorhinal cortex and hippocampus in diagnosing Alzheimer's disease. American Journal of Neuroradiology, 20, 139-144.

Kalmijn, S., Fesken, E.J.M., Launer, L.J. \& Kromhout, D. (1996). Cerebrovascular disease, the apolipoprotein e4 allele, and cognitive decline in a community-based study of elderly men. Stroke, 27, 2230-2235.

Keller, K.B. \& Lemberg, L. (1998). Conorary artery disease- ignored in women or inherently more lethal in women? American Journal of Critical Care, 7, 77-79.

Kim, J.-J., Crespo-Facorro, B., Andreasen, N.C., O'Leary, D.S., Zhang, B., Harris, G. \& Magnotta, V.A. (2000). An MRI-based parcellation method for the temporal lobe. Neuroimage, 11, 271-288.

Kivipelto, M., Helkala, E.-L., Hänninen, T., Laakso, M.P., Hallikainen, M., Alhainen, K., Soininen, H., Tuomilehto, J. \& Nissinen, A. (2001). Midlife vascular risk factors and late-life mild cognitive impairment: a population-based study. Neurology, 56, 1683-1689.

Kluger, A., Ferris, S.H., Golomb, J., Mittelman, M.S. \& Reisberg, B. (1999). Neuropsychological prediction of decline to dementia in nondemented elderly. Journal of Geriatric Psychiatry and Neurology, 12, 168-179.

Kneebone, A.C., Andrew, M.J., Baker, R.A. \& Knight, J.L. (1998). Neuropsychological changes after coronary artery surgery: use of reliable chance indices. Annals of Thoracic Surgery, 65, 1320-1325.

Knopman, D., Boland, L.L., Mosley, T., Howard, G., Liao, D., Szklo, M., McGovern, P. \& Folsom, A.R. (2001). Cardiovascular risk factors and cognitive decline in middleaged adults. Neurology, 56, 42-48.

Kolb, B. \& Wishaw, I.Q. (1993). Neuropsychologie. Heidelberg: Spektrum Akademischer Verlag.

Kramer, J.H., Reed, B.R., Mungas, D., Weiner, M.W. \& Chui, H.C. (2002). Executive dysfunction in subcortical ischaemic vascular disease. Journal of Neurology, Neurosurgery, and Psychiatry, 72, 217-220. 
Kratzmeier, H. \& Horn, R. (1987). Raven-Matrizen-Test: Standard Progressive Matrices (SPM) (Deutsche Bearbeitung). Weinheim: Beltz Test Gesellschaft.

Laakso, M.P., Partanen, K., Riekkinen, P., Lehtovirta, M., Helkala, E.-L., Hallikainen, M., Hänninen, T., Vainio, P. \& Soininen, H. (1996). Hippocampal volumes in Alzheimer's disease, Parkinson's disease, and in vascular dementia. An MRI study. Neurology, 46, 678-681.

LaBar, K.S., Gitelman, D.R., Parrish, T.B. \& Mesulam, M.-M. (1999). Neuroanatomic overlap of working memory and spatial attention networks: a functional MRI comparison within subjects. NeuroImage, 10, 695-704.

Lagerquist, B., Safstrom, K., Stahle, E., Wallentin, L. \& Swahn, E. (2001). Is early invasive treatment of unstable coronary artery disease equally effective for both women and men? FRISC II Study Group Investigators. Journal of the American College of Cardiology, 38, 41-48.

Larrabee, G.J., Largen, J.W. \& Levin, H.S. (1985). Sensitivity of age-decline resistant ("Hold") WAIS subtests to Alzheimer's disease. Journal of Clinical and Experimental Neuropsychology, 7, 497-504.

Lashley, K.S. (1938). Factors limiting recovery after central nervous lesions. Journal of Nervous and Mental Disease, 88, 733-755.

Lassmann, H., Fischer, P. \& Jellinger, K. (1993). Synaptic pathology of Alzheimer's disease. Annals of the New York Academy of Sciences, 695, 59-64.

Launer, L.J., Masaki, K., Petrovitch, H., Foley, D. \& Havlik, R.J. (1995). The association between midlife blood pressure levels and late-life cognitive function: the Honolulu-Asia Aging Study. Journal of the American Medicine Association, 274, 18461851.

Lechner, H., Schmidt, R., Bertha, G., Justich, E., Offenbacher, H. \& Schneider, G. (1988). Nuclear magnetic resonance image white matter lesions and risk factors for stroke in normal individuals. Stroke, 19, 263-265.

Leininger, B.E., Gramling, S.E., Farrell, A.D., Kreutzer, J.S. \& Peck, E.A. (1990). Neuropsychological deficits in symptomatic minor head injury patients after concussion and 
mild concussion. Journal of Neurology, Neurosurgery and Psychiatry, 53, 293296.

Lezak, M.D. (1995). Neuropsychological Assessment (Vol. 3). New York: Oxford University Press.

Lindgren, A., Roijer, A., Rudling, O., Norrvin, B., Larsson, E.-M., Eskilsson, J., Wallin, L., Olsson, B. \& Johansson, B.B. (1994). Cerebral lesions on magnetic resonance imaging, heart disease, and vascular risk factors in subjects without stroke: a population based study. Stroke, 25, 929-934.

Liu, K., Cuddy, T.E. \& Peirce, G.N. (1992). Oxidative status of lipoproteins in coronary disease patients. American Heart Journal, 123, 285-290.

Logsdon, R.G., Teri, L., Williams, D.E., Vitiello, M.V. \& Prinz, P.N. (1989). The WAIS-R profile: a diagostic tool for Alzheimer's disease? Journal of Clinical and Experimental Neuropsychology, 11, 892-898.

Longstreth, W.T., Bernick, C., Manolio, T.A., Bryan, N., Jungreis, C.A., J. \& Price, T.R. (1998). Lacunar infarcts defined by magnetic resonance imaging of 3660 elderly people: the Cardiovascular Health Study. Archives of Neurology, 55, 1217-1225.

Longstreth, W.T., Manolio, T.A., Arnold, A., Burke, G.L., Bryan, N., Jungreis, C., Enright, P.L., O'Leary, D. \& Fried, L. (1996). Clinical correlates of white matter findings on cranial magnetic resonance imaging of 3301 elderly people: the Cardiovascular Health Study. Stroke, 27, 1274-1282.

Loring, D.W., Lee, G.P., Martin, R.C. \& Meador, K.J. (1989). Verbal and Visual Memory Index discrepancies from the Wechsler Memory Scale -Revised: cautions in interpretation. Psychological Assessment, 1, 198-202.

Madden, D.J., Blumenthal, J.A., Ekelund, L.-G., Krantz, D.S., Light, K.C. \& McKee, D.C. (1986). Memory performance by mild hypertensitives follwing beta-adrenergic blockade. Psychopharmacology, 89, 20-24.

Mai, J.K., Assheuser, J. \& Paxinos, G. (1997). Atlas of the Human Brain. San Diego: Academic Press. 
Manolio, T.A., Kronmal, R.A., Burke, G.L., Poirier, V., O’Leary, D.H., Gardin, J.M., Fried, L.P., Steinberg, E.P. \& Bryan, N. (1994). Magnetic resonance abnormalities and cardiovascular disease in older adults: the Cardiovascular Health Study. Stroke, $25,318-325$.

Mäntyla, R., Aronen, H.J., Salonen, O., Pohjasvaara, T., Korpelainen, M., Peltonen, T., Standertskjöld-Nordenstam, C.-G., Kaste, M. \& Erkinjuntti, T. (1999). Magnetic resonance imaging white matter hyperintensities and mechanism of ischemic stroke. Stroke, 30, 2053-2058.

Markowitsch, H.J., Emmans, D., Irle, E., Steicher, M. \& Preilowski, B. (1985). Cortical and subcortical afferent connections of the primate's temporal pole: a study of rhesus monkeys, squirrel monkeys, and marmosets. Journal of Comparative Neurology, $242,425-458$.

Markowitsch, H.J. (1996). Neuropsychologie des menschlichen Gedächtnisses. Spektrum der Wissenschaft, 9, 52-61.

Matarazzo, J.D. (1982). Die Messung und Bewertung der Intelligenz Erwachsener nach Wechsler. Bern: Hans Huber.

Mattlar, C.-E., Engblom, E., Vesala, P., Vänttinen, E. \& Knuts, L.R. (1991). The proportion of patients with cognitive impairment after coronary artery bypass graft: an 8month follow-up study. Psychotherapy and Psychosomatic, 55, 145-150.

Maxwell, C.J., Hogan, D.B. \& Ebly, E.M. (1999). Calcium-channel blockers and cognitive function in elderly people: results from the Canadian Study of Health and Aging. Canadian Medical Association Journal, 161, 501-506.

McAllister, T.W. (1983). Overview: pseudodementia. American Journal of Psychiatry, 140, $662-664$.

McGeer, P.L., McGeer, E.G., Akiyama, H., Itagaki, S., Harrop, R. \& Peppard, R. (1990). Neuronal degeneration and memory loss in Alzheimer's disease and aging. Experimental Brain Research, 21 (Suppl), 411-426.

McKhann, G., Drachman, D., Folstein, M., Katzman, R., Price, D. \& Stadlan, E.M. (1984). Clinical diagnosis of Alzheimer's disease. Neurology, 34, 939-944. 
McKhann, G.M., Goldsborough, M.A., Borowicz, L.M., Selnes, O.A., Mellits, D.E., Enger, C., Quaskey, S.A., Baumgartner, W.A., Cameron, D.E., Stuart, R.S. \& Gardner, T.J. (1997). Cognitive outcome after coronary artery bypass: a one-year prospective study. Annals of Thoracic Surgery, 63, 510-515.

Mega, M.S. \& Cummings, J.L. (1994). Frontal-subcortical circuits and neuropsychiatric disorders. Journal of Neuropsychiatry and Clinical Neurosciences, 6, 358-370.

Meguro, K., LeMestric, C., Landeau, B., Desgranges, B., Eustache, F. \& Baron, J.C. (2001). Relation between hypometabolism in the posterior assocation neocortex and hippocampal atrophy on Alzheimer's disease: a PET/MRI correlative study. Journal of Neurology, Neurosurgery, and Psychiatry, 71, 315-321.

Miller, G.A. (1956). The magical number seven, plus or minus two: some limits on our capacity for processing information. Psychological Review, 63, 81-97.

Mirsen, T.R., Lee, D.H. \& Wong, C.J. (1991). Clinical correlates of white-matter changes on magnetic resonance imaging scans of the brain. Archives of Neurology, 48, 10151021.

Mirsky, A.F. (1989). The neuropsychology of attention: elements of a complex behavior. In E. Perecman (Ed.), Integrating theory and practice in clinical neuropsychology. New Jersey: Laurence Erlbaum.

Mittelmark, M.B., Psaty, B.M., Rautaharju, P.M., Fried, L.P., Tracey, R.P., Gardin, J.M., Borhani, N.O. \& O'Leary, D.H. (1993). Prevalence of cardiovascular diseases among older adults: the Cardiovascular Health Study. American Journal of Epidemiology, 137, 311-317.

Morán, M.A., Mufson, E.J. \& Mesulam, M.-M. (1987). Neural inputs into the temporopolar cortex of the rhesus monkey. Journal of Comparative Neurology, 256, 88-103.

Munoz, D.G., Hastak, S.M., Harper, B., Lee, D. \& Hachinski, V. (1993). Pathologic correlates of increased signals of the centrum ovale on magnetic resonance imaging. Archives of Neurology, 50, 492-497.

Murkin, J.M. (1995). The role of CPB management in neurobehavioral outcomes after cardiac surgery. Annals of Thoracic Surgery, 59, 1308-1311. 
Nemeroff, C.B., Mussleman, D.L. \& Evans, D.L. (1998). Depression and cardiac disease. Depression and Anxiety, 8 Suppl 1, 71-79.

Newman, M.F., Kirchner, J.L., Phillips-Bute, B., Gaver, V., Grocott, H., Jones, R.H., Mark, D.B., Reves, J. \& Blumenthal, J.A. (2001). Longitudinal assessment of neurocognitive function after coronary-artery bypass surgery. New England Journal of Medicine, 344, 395-402.

Nieuwenhuys, R., Voogd, J. \& Huijzen, C. van (1991). Das Zentralnervensystem des Menschen. Berlin: Springer.

Nissen, M.J. \& Bullemer, P. (1987). Attentional requirements of learning: evidence from performance measures. Cognitive Psychology, 19, 1-32.

O’Brien, D.J., Bauer, R.M., Yarandi, H., Knauf, D.G., Bramblett, P. \& Alexander, J.A. (1992). Patient memory before and after cardiac operations. Journal of Thoracic and Cardiovascular Surgery, 104, 1118-1124.

Olzewski, J. (1962). Subcortical arteriosclerotic encephalopathy. World of Neurology, 3, 359375.

Özeren, A., Acartürk, E., Koc, F., Demir, M., Sarica, Y. \& Eroglu, H. (1998). Silent cerebral lesion on magnetic resonance imaging in sujects with coronary artery disease. Japanese Heart Journal, 39, 611-618.

Pantoni, L. \& Garcia, J.H. (1995). The significance of cerebral white matter abnormalities 100 years after Binswanger's report. A review. Stroke, 26, 1293-1301.

Pascual-Leone, A., Grafman, J., Clark, K., Steward, M., Massaquoi, S., Lou, J.-S. \& Hallett, M. (1993). Procedural learning in Parkinson's disease and cerebellar degeneration. Annals of Neurology, 34, 594-602.

Perry, R.J. \& Hodges, J.R. (1999). Attention and executive deficits in Alzheimer's disease: a critical review. Brain, 122, 383-404.

Petersen, R.C., Jack, C.R., Xu, Y.-C., Waring, S.C., O’Brien, P.C., Smith, G.E., Ivnik, R.J., Tangalos, E.G., Boeve, B.F. \& Kokmen, E. (2000). Memory and MRI-based hippocampal volumes in aging and AD. Neurology, 54, 581-587. 
Petrovitch, H., White, L.R., Izmirilian, G., Ross, G.W., Havlik, R.J., Markesbery, W., Nelson, J., Davis, D.G., Harman, J., Foley, D.J. \& Launer, L.J. (2000). Midlife blood pressure and neuritic plaques, neurofibrillary tangles, and brain weight at death: the HAAS. Neurobiology of Aging, 21, 57-62.

Poeck, K. (1994). Neurologie. Berlin: Springer-Verlag.

Posner, M.I., Walker, F.J.F., Friedrich, F.A. \& Rafel, R.D. (1984). Effects of parietal injury on covert orienting of attention. Journal of Neuroscience, 4, 1863-1874.

Posner, M.I. \& Petersen, S.E. (1990). The attention system of the human brain. Annual Review of Neuroscience, 13, 25-42.

Prince, M.J., Bird, A.S., Bliziard, R.A. \& Mann, A.H. (1996). Is the cognitive function of older patients affected by antihypertensive treatment? Results from 54 months of the Medical Research Council's treatment trial of hypertension in older adults. British Medical Journal, 312, 801-805.

Pruessner, J.C., Li, L.M., Serles, W., Pruessner, M., Collins, D.L., Kabani, N., Lupien, S. \& Evans, A.C. (2000). Volumetry of hippocampus and amygdala with highresolution MRI and three-dimensional analysis software: minimizing the discrepancies between laboratories. Cerebral Cortex, 10, 433-442.

Ragland, D.R. \& Brand, R.J. (1988). Type A behavior and mortality from coronary artery disease. New England Journal of Medicine, 318, 65-69.

Reitan, R.M. (1992). Trail Making Test. Manual for administration and scoring. South Tuscon, AZ: Reitan Neuropsychological Laboratory.

Rogers, T.K. \& Bowman, C.E. (1990). Cognitive impairment associated with beta-blockade in the elderly. Postgraduate Medicine, 66, 1050-1052.

Roland, P.E. \& Zilles, K. (1998). Structural divisions and functional fields in the human cerebral cortex. Brain Research Reviews, 26, 87-105.

Rue, A. La \& Jarvik, L.R. (1987). Cognitive function and prediction of dementia in old age. International Journal of Aging and Human Development, 25, 79-89. 
Russell, E.W. (1987). Neuropsychological interpretation of the WAIS. Neuropsychology, 1, $2-6$.

Sabri, O., Ringelstein, E.-B., Hellwig, D., Schneider, R., M., S., Kaiser, H.-J., Mull, M. \& Buell, U. (1999). Neuropsychological impairment correlates with hypoperfusion and hypometabolism but not with severity of white matter lesions in MRI in patients with cerebral microangiopathy. Stroke, 30, 556-566.

Saß, H., Wittchen, H.-U. \& Zaudig, M. (1996). Diagnostisches und Statistisches Manual Psychischer Störungen DSM-IV. Göttingen: Hogrefe.

Scarpelli, M., Salvolini, U., Diamanti, L., Monitroni, R., Chiaromoni, L. \& Maricotti, M. (1994). MRI and pathological examination of post-mortem brains: the problem of white matter high signal areas. Neuroradiology, 36, 393-398.

Schanzenbächer, P. \& Kochsiek, K. (1993). Koronare Herzkrankheit. In M. Classen (Hrsg.), Innere Medizin (S. 968-981). München: Urban und Schwarzenberg.

Scheltens, P.H., Erkinjuntti, T., Leys, D., Wahlund, L.-O., Inzitari, D., Ser, T. del, Pasquier, F., Barkhof, F., Mäntylä, R., Bowlwe, J., Wallin, A., Ghika, J., Fazekas, F. \& Pantoni, L. (1998). White matter changes on CT and MRI. An overview of visual rating scales. European Neurology, 39, 80-89.

Schmidt, R., Fazekas, F., Hayn, M., Schmidt, H., Kapeller, P., Roob, G., Offenbacher, H., Schumacher, M., Eber, B., Weinrauch, V., Kostner, G.M. \& Esterbauer, H. (1997). Risk factors for microangiopathy-related cerebral damage in the Austrian stroke prevention study. Journal of Neurological Sciences, 152(1), 15-21.

Schmidt, R., Fazekas, F., Kapeller, P., Schmidt, H. \& Hartung, H.P. (1999). MRI white matter hyperintensities: three-year follow-up of the Austrian Stroke Prevention Study. Neurology, 53, 132-139.

Schmidt, R., Fazekas, F. \& Kleinert, G. (1993). Neuropsychological correlates of MRI white matter hyperintensities in the deep and white matter: a study among 150 volunteers. Neurology, 43, 2490-2494.

Schmidt, R., Fazekas, F., Koch, M., Kapeller, P., Augustin, M., Offenbach, H., Fazekas, G. \& Lechner, H. (1995). Magnetic resonance imaging cerebral abnormalities and neu- 
ropsychologic test performance in elderly hypertensive subjects. Archives of Neurology, 52, 905-910.

Schmidt, R., Fazekas, F., Offenbacher, H., Dusleag, J. \& Lechner, H. (1991). Brain magnetic resonance imaging and neuropsychological evaluation of patients with idiopathic dilated cardiomyopathy. Stroke, 22, 195-199.

Schmidt, R., Fazekas, F., Offenbacher, H., Lytwyn, H., Blematl, B., Niederkorn, K., Horner, S., Payer, F. \& Freidl, W. (1991). Magnetic resonance imaging white matter lesions and cognitive impairment in hypertensive individuals. Archives of Neurology, 48, 417-420.

Schmidt, R.F., Fazekas, F., Offenbacher, H., Mächler, H., Freidl, W., Payer, F., Rigler, B., Harrison, M.I.G. \& Lechner, H. (1993). Brain magnetic resonance imaging in coronoary artery bypass grafts: A pre- and postoperative assessment. Neurology, $43,775-778$.

Schwertz, D.W. \& Penckofer, S. (2001). Sex differences and the effect of sex hormones on hemostasis and vascular reactivity. Heart and Lung, 30, 427-428.

Scoville, W.B. \& Milner, B. (1957). Loss of recent memory after bilateral hippocampal lesion. Journal of Neurology, 20, 11-21.

Seger, C.A. (1994). Implicit learning. Psychological Bulletin, 115, 163-196.

Sellman, M., Hindmarsh, T., Ivert, T. \& Semb, B. (1992). Magnetic resonance imaging before and after open heart operations. Annals of Thoracic Surgery, 53, 807-812.

Selnes, O.A., Royall, R.M., Grega, M.A., Borowicz, L.M., Quaskey, S. \& McKhann, G.M. (2001). Cognitive changes 5 years after coronary artery bypass grafting: Is there evidence of late decline? Archives of Neurolgy, 58, 598-604.

Shaw, P.J., Bates, D., Cartlidge, N.E.F., French, J.M., Heaviside, D., Julian, D.G. \& Shaw, D.A. (1987). Long-term intellectual dysfunction follwing coronary artery bypass graft surgery: a six month follow-up study. Quarterly Journal of Medicine, 62, 259-268. 
Siesjö, B.K., Zhao, Q., Pahlmark, K., Siesjö, P., Katsura, K. \& Folbergrová, J. (1995). Glutamate, calcium, and free radicals as mediators of ischemic brain damage. Annals of Thoracic Surgery, 59, 1316-1320.

Skoog, I. (2000). Vascular aspects in Alzheimer's disease. Journal of Neural Transmission, 59 (Suppl), 37-43.

Skoog, I., Lernfelt, B., Landahl, S., Palmertz, B., Andreasson, L.A., Nilsson, L., Persson, G., Oden, A. \& Svanborg, A. (1996). 15-year longitudinal study of blood pressure and dementia. Lancet, 347, 1141-1145.

Small, B.J., Fratiglioni, L. \& Bäckman, L. (2001). Canaries in a coal mine: cognitive markers of preclinical Alzheimer disease. Archives of General Psychiatry, 58, 859-860.

Sohlberg, M.M. \& Mateer, C.A. (1987). Effectiveness of an attention training program. Journal of Clinical and Experimental Neuropsychology, 9, 117-130.

Soneira, C.F. \& Scott, T.M. (1996). Severe cardiovascular disease and Alzheimer's disease: Senile plaque formation in cortical areas. Clinical Anatomy, 9, 118-127.

Sparks, D.L., Hunsaker, J.C., Scheff, S.W., Kryscio, R.J., Henson, J.L. \& Markesbery, W.R. (1990). Cortical senile plaques in coronary artery disease, aging and Alzheimer's disease. Neurobiology of Aging, 11, 601-607.

Sparks, D.L., Liu, H., Scheff, S.W., Coyne, C.M. \& Hunsaker, J.C. (1993). Temporal sequence of plaque formation in the cerebral cortex of nondemented individuals. Journal of Neuropathology and Experimental Neurology, 52, 135-142.

Sparks, D.L., Martin, T.A., Gross, D.R. \& Hunsaker, J.C. (2000). Link between heart disease, cholesterol, and Alzheimer's disease: a review. Microscopy Research and Technique, 50, 287-290.

Sparks, D.L., Scheff, S.W., Liu, H., Landers, T.M., Coyne, C.M. \& Hunsacker, J.C. (1995). Increased incidence of neurofibrillary tangles (NFT) in non-demented individuals with hypertension. Journal of the Neurological Sciences, 131, 162-169.

Squire, L.R. (1986). Mechanisms of memory. Science, 232, 1612-1619. 
Squire, L.R. \& Shimamura, A.P. (1986). Characterizing amnesic patients for neurobehavioral study. Behavioral Neuroscience, 100, 866-877.

Steingart, A., Hachinski, V.C., Lau, C., Fox, A.J., Diaz, F., Cape, R., Lee, D., Inzitari, D. \& Merskey, H. (1987). Cognitive and neurologic findings in subjects with diffuse white matter lucencies on computed tomographic scan (leuko-araiosis). Archives of Neurology, 44, 32-35.

Sullivan, E.V., Corkin, S. \& Growdon, J.H. (1986). Verbal and nonverbal short-term memory in patients with Alzheimer's disease and in healthy elderly subjects. Developmental Neuropsychology, 2, 387-400.

Sullivan, P., Pary, R., Telang, F., Rifai, A.H. \& Zubenko, G.S. (1990). Risk factors for white matter changes detected by magnetic resonance imaging in the elderly. Stroke, 21, 1424-1428.

Swieten, J.C. Van, Geykes, G.G. \& Derix, M.M.A. (1991). Hypertension in the elderly is associated with white matter lesions and cognitive decline. Annals of Neurology, 30, 825-830.

Tanizaki, Y., Kiyohara, Y., Kato, I., Iwamoto, H., Nakayama, K., Shinohara, N., Arima, H., Tanaka, K., Ibayashi, S. \& Fujishima, M. (2000). Incidence and risk factors for subtypes of cerebral infarction in a general population: The Hisayama Study. Stroke, 31, 2616-2622.

Tewes, U. (1991). HAWIE-R - Hamburg-Wechsler-Intelligenztest für Erwachsene - Revision 1991. Bern: Hans Huber.

Tulving, E. (1984). How many memory systems are there? American Psychologist, 40, 385398.

Tupler, L.A., Coffey, C.E., Logue, P.E., Djang, W.T. \& Fagan, S.M. (1992). Neuropsychological importance of subcortical white matter hyperintensity. Archives of Neurology, 49, 1248-1252.

Tzourio, C., Dufouil, C., Ducimetière, P. \& Alpérovitch, A. (1999). Cognitive decline in individuals with high blood pressure: a longitudinal study in the elderly. Neurology, $53,1948-1952$. 
Vakil, E., Hoofien, D. \& Blachstein, H. (1992). Total amount learned versus learning rate of verbal and nonverbal information, in differentiating left- from right-brain injured patients. Archives of Clinical Neuropsychology, 12, 587-596.

Veldink, J.H., Scheltens, P., Jonker, C. \& Launer, L.J. (1998). Progression of cerebral white matter hyperintensities on MRI is related to diastolic blood pressure. Neurology, $51,319-320$.

Vik, A., Brubakk, A.O., Rinck, P.A., Levang, O.W. \& Sellevold, O. (1991). MRI: a method to detect minor brain damage following coronary bypass surgery. Neuroradiology, 33, 396-398.

Visser, P.J., Verhey, F.R.J., Hofman, P.A.M., Scheltens, P. \& Jolles, J. (2002). Medial temporal lobe atrophy predicts Alzheimer's disease in patients with minor cognitive impairment. Journal of Neurology, Neurosurgery, and Psychiatry, 72, 491-497.

Volicer, L. \& Crino, P.B. (1990). Involvement of free radicals in dementia of the Alzheimer type: a hypothesis. Neurobiology of Aging, 11, 567-571.

Walzer, T., Herrmann, M. \& Wallesch, C.-W. (1997). Neuropsychological disorders after coronary bypass surgery. Journal of Neurology, Neurosurgery, and Psychiatry, 62, 644-648.

Warrington, E.K., James, M. \& Maciejewski, C. (1986). The WAIS as a lateralizing and localizing diagnostic instrument. Neuropsychologia, 24, 223-239.

Wechsler, D. (1955). Manual for the Wechsler Adult Intelligence Scale. New York: Psychological Corporation.

Wechsler, D. (1981). Wechsler Adult Intelligence Scale - Revised. New York: Psychological Corporation.

Wechsler, D. (1987). Wechsler Memory Scale-Revised manual. San Antonio, TX: Psychological Corporation.

Wityk, R.J., Goldsborough, M.A., Hillis, A., Beauchamp, N., Barker, P.B., Borowicz, L.M. \& McKhann, G.M. (2001). Diffusion- and perfusion-weighted brain magnetic resonance imaging in patients with neurologic complications after cardiac surgery. Archives of Neurology, 58, 571-576. 
Wolf, H., Ecke, G.M., Bettin, S., Dietrich, J. \& Gertz, H.-J. (2000). Do white matter changes contribute to the subsequent development of dementia in patients with mild cognitive impairment? International Journal of Geriatric Psychiatry, 15, 803-812.

Yamaguchi, S., Meguro, K., Shimada, M., Ishizaki, J., Yanadori, A. \& Sekita, Y. (2002). Five-year retrospective changes in hippocampal atrophy and cognitive screening test performances in very mild Alzheimer's diease: the Tajiri Project. Neuroradiology, 44, 43-48.

Yao, H., Sadoshima, S., Ibayashi, S., Kuwabara, Y., Ichiya, Y. \& Fujishima, M. (1992). Leukoraiosis and dementia in hypertensive patients. Stroke, 23, 1673-1677.

Ylikoski, R., Ylikoski, A., Erkinjuntti, T., Sulkava, R., Rainiko, R. \& Tilvis, R. (1993). White matter changes in healthy elderly persons correlate with attention and speed of mental processing. Archives of Neurology, 50, 818-824.

Zimmerman, I.L., Woo-Sam, J.W. \& Glasser, A.J. (1973). Clinical interpretation of the Wechsler Adult Intelligence Scale. New York: Grune \& Stratton.

Zimmermann, P. \& Fimm, B. (1993). Testbatterie zur Aufmerksamkeitsprüfung. Freiburg: Psytest.

Zomeren, A.H. van \& Burg, W. van den (1985). Residual complaints of patients two years after severe brain injury. Journal of Neurology, Neurosurgery and Psychiatry, 48, 21-28. 
Die Universitätsklinik Göttingen bemüht sich, ihre eingesetzten Behandlungsmethoden und Operationstechniken wissenschaftlich zu überprüfen und dem Erkenntnisstand der Medizin anzupassen. Momentan werden in einer Zusammenarbeit der Abteilung Thorax-, Herz- und Gefäßchirurgie bzw. Kardiologie und der Neuropsychologie Herz-Patienten in einer Studie untersucht. Dabei kommen bestimmte Verfahren zum Einsatz, die die Leistungsfähigkeit des Gehirns untersuchen, z.B. bezüglich Aufmerksamkeit, Reaktionsgeschwindigkeit und Gedächtnis. Die Untersuchung dauert insgesamt ca. 5 Stunden. Neben der neuropsychologischen Untersuchung werden mit einem modernen bildgebenden Verfahren, der Magnetresonanztomographie, Aufnahmen des Schädels angefertigt. Diese Untersuchung dient dazu, Aufschlüsse über die Struktur und Beschaffenheit des Gehirns zu bekommen. Die Untersuchung ist absolut ungefährlich. Es wird nicht mit Strahlen, sondern mit einem starken Magnetfeld gearbeitet. Aufgrund dieses starken Magnetfeldes darf diese Untersuchung jedoch nicht bei Personen durchgeführt werden, bei denen sich Metallteile (z.B. Herzschrittmacher, Metallplatten, Klammern) im Körper befinden. Die Magnetresonanztomographie wird ca. 15 Minuten dauern. Sie werden in einer relativ engen Röhre, aber trotzdem bequem, gelagert werden. Durch das Magnetfeld entstehen Geräuscheinwirkungen, die jedoch durch Ohrstöpsel gedämpft werden. Sie haben jederzeit die Möglichkeit, über Mikrophon und Kopfhörer mit dem Untersuchungspersonal zu sprechen.

Alle personenbezogenen Daten werden gemäß den geltenden rechtlichen Bestimmungen (§203 StGB) vor unbefugter Offenbarung strikt geschützt. Auf Ihren Wunsch können die Ergebnisse der Untersuchungen jedoch Ihrem nachbehandelnden Arzt (Hausarzt, Facharzt) zur Verfügung gestellt werden. Für Sie selbst entstehen durch die Teilnahme an der Studie keinerlei Kosten, so werden z.B. die Fahrtkosten erstattet.

Die Teilnahme an dieser Studie ist unverbindlich, und selbstverständlich können Sie Ihre Teilnahme an der Studie zu jedem Zeitpunkt ohne Angabe von Gründen widerrufen. Über Ihre Bereitschaft würden wir uns jedoch sehr freuen und stehen für weitere Fragen Ihrerseits zur Verfügung.

Ihre Ansprechpartnerin ist Frau Dipl.-Psych. Koschack. 


\section{SERIENBRIEF FÜR DIE REKRUTIERUNG DER AMBULAN- TEN PATIENTEN}

Sehr geehrter «Anrede» «Name»,

Sie sind vor kurzem in der Kardiologischen Klinik der Universität Göttingen behandelt worden.

Die Universitätskliniken bemühen sich um eine möglichst umfassende Nachuntersuchung aller betreuten Patienten. Vor diesem Hintergrund findet in der neuropsychologischen Abteilung der Universität Göttingen derzeit eine wissenschaftliche Studie statt. In dieser Studie werden Patienten mit Ihrem Krankheitsbild untersucht. Dabei kommen neuropsychologische Testverfahren zum Einsatz, mit denen verschiedene Funktionen des Gehirns erfaßt werden können, z.B. Aufmerksamkeit, Reaktionsgeschwindigkeit und Gedächtnis. Neben dieser neuropsychologischen Untersuchung werden mit einem modernen bildgebenden Verfahren, der Magnetresonanztomographie, Schichtaufnahmen des Schädels angefertigt. Die Teilname an der Studie wäre für Sie mit einem zeitlichen Aufwand von ca. 5 Stunden verbunden, Kosten entstehen natürlich keine für Sie, eventuelle Fahrtkosten werden erstattet. Die Ergebnisse der Untersuchung werden auf Wunsch selbstverständlich Ihren behandelnden Ärzten zur Verfügung gestellt.

Wir werden uns in nächster Zeit telephonisch mit Ihnen in Verbindung setzen, um Ihr Einverständnis zu erfragen und ggf. einen Termin auszumachen. Bei Nachfragen können Sie sich aber auch selbst telephonisch in der Zeit von Mo-Frei jeweils zwischen 9.00 und 16.00 Uhr unter der Nummer 0551/ 39-5990 an uns wenden. Briefe erreichen uns unter der Anschrift, die Sie dem Briefkopf entnehmen können.

Für Ihre Mitarbeit wären wir Ihnen sehr dankbar und verbleiben mit freundlichen Grüßen, 


\section{WISSENSCHAFTLICHER BILDUNGSGANG DER VERFAS- SERIN}

\author{
ANGABEN ZUR PERSON \\ Name: $\quad$ Janka Koschack \\ geboren: $\quad 03.11 .1972$ in Delmenhorst \\ Familienstand: verheiratet \\ Nationalität: deutsch \\ $1979-1983$ \\ 1983-1985 \\ 1985-1992

\section{SCHULBILDUNG} \\ Hermann-Allmers-Grundschule in Delmenhorst \\ Hermann-Allmers-Orientierungsstufe in Delmenhorst \\ Gymnasium an der Max-Planck-Straße in Delmenhorst
}

$1992-1993$

1993-1995

1995-1998

seit 1999

\section{HOCHSCHULAUSBILDUNG}

Biologie-Studium an der Georg-August-Universität Göttingen

Psychologie-Studium an der Technischen Universität Berlin

Abschluß: Vordiplom

Psychologie-Studium an der Georg-August-Universität Göttingen

Abschluß: Hauptdiplom

\section{BERUFLICHE TÄTIGKEIT}

Wissenschaftliche Angestellte an der Klinik und Poliklinik für

Psychiatrie und Psychotherapie der Universität Göttingen, Schwerpunkt Psychopathologie und Neuropsychologie 